\title{
A SPITZER c2d LEGACY SURVEY TO IDENTIFY AND CHARACTERIZE DISKS WITH INNER DUST HOLES
}

\author{
Bruno Merín ${ }^{1,3}$, Joanna M. Brown ${ }^{2}$, Isa Oliveira ${ }^{3}$, Gregory J. Herczeg ${ }^{2}$, Ewine F. van Dishoeck ${ }^{2,3}$, \\ Sandrine Bottinelli ${ }^{4}$, Neal J. Evans II $^{5}$, Lucas Cieza ${ }^{6}$, Loredana Spezzi ${ }^{7}$, Juan M. Alcalá ${ }^{8}$, Paul M. Harvey ${ }^{5}$, \\ Geoffrey A. Blake ${ }^{9}$, Amelia Bayo ${ }^{10}$, Vincent G. Geers ${ }^{11}$, Fred Lahuis ${ }^{3,12}$, Timo Prusti ${ }^{7}$, Jean-Charles Augereau ${ }^{4}$, \\ Johan Olofsson ${ }^{4}$, Frederick M. Walter ${ }^{13}$, And Kuenley Chiu ${ }^{14}$ \\ ${ }^{1}$ Herschel Science Centre, European Space Astronomy Centre (ESA), P.O. Box 78, 28691 Villanueva de la Cañada (Madrid), Spain \\ ${ }^{2}$ Max-Planck-Institut für Extraterrestrische Physik, Giessenbachstraße, 85748 Garching bei München, Germany \\ ${ }^{3}$ Leiden Observatory, Leiden University, P.O. Box 9513, 2300 RA Leiden, The Netherlands \\ ${ }^{4}$ Laboratoire d'Astrophysique de Grenoble, Université Joseph Fourier, CNRS, UMR 5571, Grenoble, France \\ ${ }^{5}$ Department of Astronomy, University of Texas at Austin, 1 University Station, C1400 Austin, TX 78712-0259, USA \\ ${ }^{6}$ Institute for Astronomy, University of Hawaii at Manoa, Honolulu, HI 96822, USA \\ ${ }^{7}$ RSSD, European Space Agency (ESTEC), P.O. Box 299, 2200 AG Noordwijk, The Netherlands \\ 8 INAF-Osservatorio Astronomico di Capodimonte, Salita Moiariello 16, 80131 Napoli, Italy \\ ${ }^{9}$ Division of Geological and Planetary Sciences, MS 150-21, California Institute of Technology, Pasadena, CA 91125, USA \\ ${ }^{10}$ European Southern Observatory, Alonso de Cordova 3107, Vitacura, Santiago, Chile \\ ${ }^{11}$ Astronomy Department, University of Toronto, Ontario, Canada \\ ${ }^{12}$ SRON Netherlands Institute for Space Research, P.O. Box 800, 9700 AV Groningen, The Netherlands \\ ${ }^{13}$ Department of Physics and Astronomy, Z-3800, Stony Brook University, Stony Brook, NY 11794-3800, USA \\ ${ }^{14}$ Department of Astronomy, MS 249-17, California Institute of Technology, Pasadena, CA 91125, USA \\ Received 2009 December 31; accepted 2010 June 7; published 2010 July 13
}

\begin{abstract}
Understanding how disks dissipate is essential to studies of planet formation. However, identifying exactly how dust and gas dissipate is complicated due to the difficulty of finding objects that are clearly in the transition phase of losing their surrounding material. We use Spitzer Infrared Spectrograph (IRS) spectra to examine 35 photometrically selected candidate cold disks (disks with large inner dust holes). The infrared spectra are supplemented with optical spectra to determine stellar and accretion properties and $1.3 \mathrm{~mm}$ photometry to measure disk masses. Based on detailed spectral energy distribution modeling, we identify 15 new cold disks. The remaining 20 objects have IRS spectra that are consistent with disks without holes, disks that are observed close to edge-on, or stars with background emission. Based on these results, we determine reliable criteria to identify disks with inner holes from Spitzer photometry, and examine criteria already in the literature. Applying these criteria to the $\mathrm{c} 2 \mathrm{~d}$ surveyed starforming regions gives a frequency of such objects of at least $4 \%$ and most likely of order $12 \%$ of the young stellar object population identified by Spitzer. We also examine the properties of these new cold disks in combination with cold disks from the literature. Hole sizes in this sample are generally smaller than in previously discovered disks and reflect a distribution in better agreement with exoplanet orbit radii. We find correlations between hole size and both disk and stellar masses. Silicate features, including crystalline features, are present in the overwhelming majority of the sample, although the $10 \mu \mathrm{m}$ feature strength above the continuum declines for holes with radii larger than $\sim 7 \mathrm{AU}$. In contrast, polycyclic aromatic hydrocarbons are only detected in 2 out of 15 sources. Only a quarter of the cold disk sample shows no signs of accretion, making it unlikely that photoevaporation is the dominant hole-forming process in most cases.
\end{abstract}

Key words: planetary systems - protoplanetary disks - stars: pre-main sequence

Online-only material: color figures

\section{INTRODUCTION}

Near- and mid-infrared observations of young stars demonstrate that optically thick circumstellar disks disappear around approximately half of low-mass young stars in 1-3 Myr and are nearly entirely absent around members of $10 \mathrm{Myr}$ old associations (e.g., Haisch et al. 2001; Gutermuth et al. 2004; Sicilia-Aguilar et al. 2005; Low et al. 2005; Currie et al. 2009). Accretion ceases on approximately the same timescale (e.g., Calvet et al. 2005). The disappearance of gas and dust-planetary building material-places stringent limits on the timescales of giant planet formation (Pollack et al. 1996; Kenyon \& Bromley 2009). However, identifying exactly how dust and gas dissipate is complicated due to the difficulty of identifying objects that are clearly in the transition phase of losing their surrounding material.
One of the most common methods, dating back to IRAS (e.g., Strom et al. 1989; Skrutskie et al. 1990), to identify candidate transitional systems between the classical and debris disk evolutionary stages is through mid-IR spectral energy distributions (SEDs). Dust growth, sedimentation, and removal are expected to result in a deficit of infrared flux as material is accreted or dispersed. This deficit has become the defining characteristic of transitional disks. For some disks, flux deficits are seen at all wavelengths, suggesting a gradual dissipation of mass for all disk radii. In other cases, a flux deficit is only seen at short wavelengths, indicating that the outer disk remains massive and optically thick, while the inner disk lacks small dust grains. The presence of both types of transitional disks suggests that the transition happens via different paths from the gas-rich, optically thick stage to gas-poor, optically thin disks (Cieza et al. 2007; Currie et al. 2009). The fraction of stars with transitional 
disks is thought to be 5\%-25\% (Lada et al. 2006; Hernández et al. 2007; Sicilia-Aguilar et al. 2008; Dahm \& Carpenter 2009; Currie et al. 2009; Kim et al. 2009). The small numbers indicate that the evolutionary path through a transitional disk is either uncommon or rapid. However, a consensus in nomenclature is still lacking: even within a single cluster, the calculated fraction of transitional disks can range from $10 \%$ to $50 \%$ depending on definitions (Ercolano et al. 2009; Sicilia-Aguilar et al. 2008).

Transition disks with a short-wavelength deficit are particularly interesting because they are potential tracers of planet formation. This deficit arises from the absence of hot small dust grains close to the star, resulting in flux coming solely from the stellar photosphere, rather than disk surface emission. This deficit may be the result of grain growth and sedimentation but also of complex interactions between a nascent protoplanet and the surrounding disk. The flux deficit from such inner holes results in a depressed SED at wavelengths less than $\sim 15 \mu \mathrm{m}$, while retaining typical fluxes at longer wavelengths from the outer disk. The short-wavelength flux deficit arises from the absence of hot small dust grains close to the star resulting in flux coming solely from the stellar photosphere, rather than disk surface emission. We define such disks as "cold disks" to differentiate them from other types of transitional systems (e.g., anemic or homologously depleted, defined as disks with an overall lower mass at all radii) and to emphasize that this is an observational characteristic not necessarily tied to evolution.

SEDs, relying heavily on mid-IR spectroscopy, are the tool currently most widely used to infer the presence of holes and gaps (e.g., Calvet et al. 2002; Forrest et al. 2004; Brown et al. 2007, hereafter B07). Additional submillimeter observations of the most massive disks have directly imaged large holes (Piétu et al. 2006; Hughes et al. 2007, 2009; Brown et al. 2008, 2009; Andrews et al. 2009). The hole sizes match reasonably well with estimates from SED modeling, suggesting that the current interpretation and modeling of SEDs are correct. Within the category of cold disks, significant differences are seen in hole sizes (from 1 to $50 \mathrm{AU}$ ), the presence or absence of gas and dust within the hole, and the presence or absence of accretion (Najita et al. 2007; Espaillat et al. 2007; Pontoppidan et al. 2008; Salyk et al. 2009; Kim et al. 2009). Detailed comparisons of these properties with the predictions of each of the scenarios listed above can help us discriminate which processes control the observed disk evolution. However, statistical studies (e.g., Najita et al. 2007) are limited by small sample sizes. Spitzer mapping efforts should include a significant number of unidentified cold disks and other types of transitional disks. However, accurately identifying these cold disks out of the large numbers of stars present in the maps is difficult with just broadband photometry. Reliable criteria to identify disks with inner holes from Spitzer photometry, which lacks any information on fluxes in the crucial region between 8 and $24 \mu \mathrm{m}$, are needed to generate a large sample for statistical purposes.

This paper presents a new large sample of cold disks identified from the "Cores to Disks" data (Evans et al. 2009) and simultaneously analyzes follow-up observations of the sample with the Infrared Spectrograph (IRS) and MIPS instruments together with optical spectroscopy and millimeter continuum observations. It is organized as follows: Section 2 describes the target selection, observations, and data reduction of the different data sets, including the Spitzer, optical spectroscopy, and millimeter observations. The results are presented in Sections 3 and 4, describing, in order, the stellar parameters in Section 3, disk masses in Section 4.1, disk parameters in Section 4.2, and dust mineralogy in Section 4.3 for the whole sample. Notes on individual sources are given in Section 4.4. The combined analysis is presented in Section 5, first with the presentation of a new selection criteria for transitional disks in Section 5.1, then a description of the observational properties of our cold disks in Section 5.2, and finally a discussion on the possible origins of the inner holes in Section 5.3. Conclusions are given in Section 6.

\section{OBSERVATIONS AND DATA REDUCTION}

\subsection{Target Selection}

The c2d Spitzer Legacy program completed a full Infrared Array Camera (IRAC; 3.6-8 $\mu \mathrm{m}$ ) and MIPS (24-160 $\mu \mathrm{m})$ survey of five nearby star-forming regions (Perseus, Chameleon II, Lupus, Ophiuchus, and Serpens; Evans et al. 2003; Jørgensen et al. 2006; Alcalá et al. 2008; Merín et al. 2008; Harvey et al. 2007). From these maps, 1024 young stellar object (YSO) candidates were identified using the selection techniques, including removing extragalactic contaminants, described in Harvey et al. (2007; see Evans et al. 2009 for a complete description of this sample and a general study of cloud-to-cloud differences). This sample should include a significant number of cold disks but they must first be separated from the bulk of the population.

Cold disks were selected for IRS follow-up using the following method. Spectral types were taken from the literature where possible; otherwise, a K7 photosphere was used to get an initial estimate of the SED. Sources with photospheric fluxes in at least IRAC $1(3.6 \mu \mathrm{m})$ and IRAC $2(4.5 \mu \mathrm{m})$ and $24 \mu \mathrm{m}$ excesses equal to or greater than IRAC $4(8.0 \mu \mathrm{m})$ fluxes in $\lambda F_{\lambda}$ space were selected. Sources with rising or flat SEDs from $8 \mu \mathrm{m}$ to $24 \mu \mathrm{m}$ were preferentially included. A flux lower limit of $15 \mathrm{mJy}$ at $8 \mu \mathrm{m}$ was imposed to further reduce extragalactic contamination and keep integration times reasonable. The initially selected sample was then cut slightly to reject likely contaminants and to avoid overlap with other IRS programs. A total of 33 sources were selected in this fashion. To further expand our sample, two additional candidate cold disks within the $\mathrm{c} 2 \mathrm{~d}$ clouds were included from a survey of Weak-line T Tauri stars (WTTs; Padgett et al. 2006; Cieza et al. 2007) based on their IRS spectra suggestive of inner holes. In total, 35 objects were included in our sample and followed up with IRS and MIPS (Section 2.2), optical spectroscopy (Section 2.3), and millimeter continuum observations (Section 2.4).

\subsection{Spitzer IRS and MIPS Observations}

Spectra for the 35 objects in Table 1 were obtained using IRS aboard the Spitzer Space Telescope under a variety of programs. Most were observed as part of program 30843 (PI: B. Merín), which also included deep $70 \mu \mathrm{m}$ MIPS staring observations of the same sample. Objects 17 and 19 were observed with IRS as part of the c2d second look observations of WTTs from Padgett et al. (2006), and object 25 was observed during the IRS Guaranteed Time Observation (GTO) although it was selected as a candidate from the $\mathrm{c} 2 \mathrm{~d}$ photometry. All the candidate cold disks in Serpens were observed as part of a complete flux-limited IRS survey in Serpens, program 30223 (PI: K. M. Pontoppidan), and are also discussed along with the rest of the Serpens sample in Oliveira et al. (2010). Table 1 gives the AOR (Astronomical Observation Request) identifications and observing dates for both the IRS and MIPS-70 observations of the sample.

All of the IRS spectra were obtained with a combination of Short-Low (SL) and Long-Low (LL) modules, which provide a 
Table 1

Cold Disk Candidates and Spitzer Observing Log

\begin{tabular}{|c|c|c|c|c|c|c|c|c|c|c|}
\hline \multirow[t]{2}{*}{ ID } & \multirow[t]{2}{*}{ Name } & \multirow[t]{2}{*}{ Cloud } & \multirow[t]{2}{*}{$\alpha_{K-24}$} & \multirow[t]{2}{*}{$\alpha_{8-24}$} & \multirow[t]{2}{*}{ Program $^{\mathrm{a}}$} & \multirow[t]{2}{*}{ Refs. } & \multicolumn{2}{|c|}{ IRS } & \multicolumn{2}{|c|}{ MIPS-70 } \\
\hline & & & & & & & AOR & Obs. Date & AOR & Obs. Date \\
\hline 1 & SSTc2d J032835.1+302010 & Per & 0.15 & 2.13 & 1 & 1,15 & 19059712 & 2007 Mar 18 & 19954688 & 2007 Feb 3 \\
\hline 2 & ASR 118 & Per & -0.22 & 0.77 & 1 & 2,15 & 19056128 & 2006 Sep 19 & 19057664 & 2007 Sep 20 \\
\hline 3 & SSTc2d J032903.9+305630 & Per & -0.17 & 1.25 & 1 & 15 & 19954176 & 2007 Mar 19 & 19954688 & $2007 \mathrm{Feb} 3$ \\
\hline 4 & SSTc2d J032924.1+311958 & Per & 0.36 & 1.57 & 1 & 1,15 & 19052544 & 2007 Mar 22 & 19057664 & 2007 Sep 20 \\
\hline 5 & SSTc2d J032929.3+311835 & Per & -0.66 & 1.30 & 1 & 2,15 & 19053312 & 2007 Mar 9 & 19057664 & 2007 Sep 20 \\
\hline 6 & SSTc2d J033027.2+302830 & Per & 0.00 & 2.37 & 1 & 1,15 & 19953920 & 2007 Mar 9 & 19057664 & 2007 Sep 20 \\
\hline 7 & SSTc2d J033035.5+311559 & Per & -0.19 & 2.04 & 1 & 15 & 19053056 & 2007 Mar 9 & 19954688 & 2007 Feb 3 \\
\hline 8 & SSTc2d J033038.2+303212 & Per & 0.35 & 2.98 & 1 & 15 & 19953664 & 2007 Mar 19 & 19057664 & 2007 Sep 20 \\
\hline 9 & LZK 21 & Per & -0.66 & 0.06 & 1 & 3,15 & 19052032 & 2007 Mar 9 & 19954688 & 2007 Feb 23 \\
\hline 10 & SSTc2d J034227.1+314433 & Per & -0.93 & 0.74 & 1 & 15 & 19053568 & 2007 Mar 18 & 19954432 & 2007 Sep 18 \\
\hline 11 & Cl* IC 348 LRL 190 & Per & -1.00 & 0.05 & 1 & $4,6,7,15$ & 19054848 & 2007 Mar 18 & 19954432 & 2007 Sep 18 \\
\hline 12 & SSTc2d J034434.8+315655 & Per & -1.11 & 0.65 & 1 & $4,5,6,15$ & 19053824 & 2007 Mar 15 & 19954432 & 2007 Sep 18 \\
\hline 13 & Cl* IC 348 LRL 265 & Per & -0.09 & 0.33 & 1 & 15 & 19052288 & 2006 Sep 18 & 19954432 & 2007 Sep 18 \\
\hline 14 & Cl* IC 348 LRL 110 & Per & -0.60 & 0.50 & 1 & $4,6,7,15$ & 19052288 & 2006 Sep 18 & 19954432 & 2007 Sep 18 \\
\hline 15 & $\mathrm{LkH} \alpha 329$ & Per & -1.02 & -0.24 & 1 & 8,15 & 19056384 & 2007 Mar 9 & 19954432 & 2007 Sep 18 \\
\hline 16 & Hn 24 & Cha & -0.85 & -0.16 & 1 & 9,16 & 19054592 & 2007 Mar 11 & 19058944 & 2006 Aug 15 \\
\hline 17 & Sz 84 & Lup & -2.34 & -0.49 & 2 & 10,19 & 05644288 & 2004 Mar 25 & 5697024 & 2005 Mar 7 \\
\hline 18 & SSTc2d J161029.6-392215 & Lup & -1.07 & 0.01 & 1 & 17 & 19051008 & 2007 Mar 19 & 19055360 & 2006 Aug 18 \\
\hline 19 & RX J1615.3-3255 & Oph & -1.64 & -0.10 & 2 & 11,19 & 15916800 & 2005 Sep 9 & 5703424 & 2005 Mar 7 \\
\hline 20 & SSTc2d J162148.5-234027 & Oph & -0.33 & 0.32 & 1 & 18 & 15920896 & 2005 Sep 9 & $\ldots$ & $\ldots$ \\
\hline 21 & SSTc2d J162221.1-230403 & Oph & 0.93 & 1.43 & 1 & 18 & 15920896 & 2005 Sep 9 & $\ldots$ & $\ldots$ \\
\hline 22 & SSTc2d J162245.4-243124 & Oph & -0.83 & 0.69 & 1 & 18 & 15920641 & 2006 Mar 15 & $\ldots$ & $\ldots$ \\
\hline 23 & SSTc2d J162332.9-225847 & Oph & -1.07 & -0.37 & 1 & 18 & 15920641 & 2006 Mar 15 & $\ldots$ & $\ldots$ \\
\hline 24 & SSTc2d J162506.9-235050 & Oph & -1.10 & 0.68 & 1 & 18 & 19059200 & 2007 Mar 19 & 19954944 & 2006 Sep 1 \\
\hline 25 & DoAr 21 & Oph & -1.16 & -0.43 & 3 & $12,13,18$ & 12699392 & 2006 Mar 15 & $\ldots$ & $\cdots$ \\
\hline 26 & SSTc2d J182829.1+002756 & Ser & -0.67 & -0.11 & 4 & 20 & 17885184 & 2007 Apr 24 & $\cdots$ & $\cdots$ \\
\hline 27 & SSTc2d J182858.1+001724 & Ser & -1.92 & -2.97 & 4 & 20 & 17888768 & 2007 May 5 & $\ldots$ & $\ldots$ \\
\hline 28 & SSTc2d J182907.0+003838 & Ser & -0.75 & -1.48 & 4 & 20 & 17884672 & 2007 Apr 28 & $\ldots$ & $\ldots$ \\
\hline 29 & SSTc2d J182911.5+002039 & Ser & -0.85 & 0.03 & 4 & 20 & 17887744 & 2007 Apr 24 & $\ldots$ & $\cdots$ \\
\hline 30 & SSTc2d J182915.6+003912 & Ser & -0.31 & -1.21 & 1 & 20 & 17884672 & 2007 Apr 28 & 19057152 & 2007 May 18 \\
\hline 31 & SSTc2d J182915.6+003923 & Ser & -0.47 & -0.73 & 1 & 20 & 17885440 & 2007 Apr 24 & $\ldots$ & $\cdots$ \\
\hline 32 & SSTc2d J182935.6+003504 & Ser & -1.02 & -0.40 & 1 & 20 & 17884160 & 2007 Apr 19 & 19057152 & 2007 May 18 \\
\hline 33 & SSTc2d J182936.2+004217 & Ser & -0.68 & -0.32 & 4 & 20 & 17884160 & 2007 Apr 19 & $\ldots$ & $\ldots$ \\
\hline 34 & SSTc2d J182944.1+003356 & Ser & -1.41 & -1.05 & 4 & 20 & 17886720 & 2007 Apr 24 & $\ldots$ & $\ldots$ \\
\hline 35 & SSTc2d J182947.3+003223 & Ser & -0.90 & -1.50 & 1 & 20 & 17887488 & 2007 Apr 25 & 19057152 & 2007 May 18 \\
\hline
\end{tabular}

Notes.

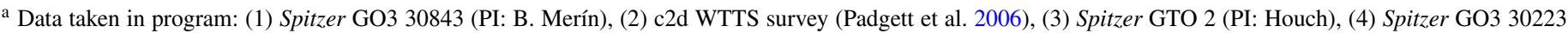
(PI: K. M. Pontoppidan), (5) c2d CTTS IRS survey (Kessler-Silacci et al. 2006).

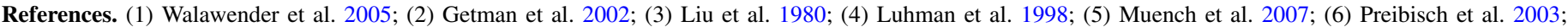

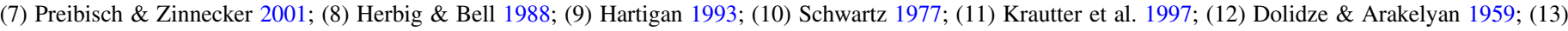

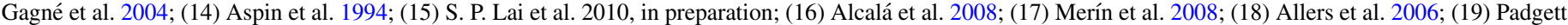
et al. 2006; (20) Harvey et al. 2007; (21) Brown et al. 2007; (22) Brown et al. 2008; (23) Kessler-Silacci et al. 2006.

resolving power of $R=\Delta \lambda / \lambda \sim 100$ and a combined wavelength coverage from 5.3 to $35.0 \mu \mathrm{m}$. Integration times were estimated from the IRAC flux at $8 \mu \mathrm{m}$ and MIPS flux at $24 \mu \mathrm{m}$. The observations typically ranged from 5 to 20 minutes, resulting in signal-to-noise ratio $(\mathrm{S} / \mathrm{N})$ values of 30-150, depending on the background level and the brightness of the objects. Given the rising SED of the targets at long wavelengths, longer times were usually needed in the SL than LL observations. The observations were scheduled in cluster mode, grouping nearby sources with similar integration times to increase the observing efficiency. Data reduction started from the Basic Calibrated Data (BCD) images, pipeline version S12.4.0. The processing includes bad-pixel correction, extraction, defringing, and order matching using the c2d analysis pipeline (Kessler-Silacci et al. 2006; also see the c2d Spectroscopic Explanatory Supplement from the Spitzer Science Center (SSC) Web site ${ }^{15}$ ). The final spectra can be found in Figures 1-5.

\footnotetext{
15 http://ssc.spitzer.caltech.edu/spitzermission/observingprograms/legacy/c2d/
}

The same clustering strategy was used for the MIPS observations at $70 \mu \mathrm{m}$. The integration times were set to $300 \mathrm{~s}$, with achieved sensitivities given in Table 2 . The small maps were reduced following the procedure described in Cieza et al. (2008) and using the SSC median-filtered BCDs from the SSC pipeline version S16.1.0. Details of the method can be found in the aforementioned paper, but, briefly, the photometry is determined with an aperture of $16^{\prime \prime}$ radius and a sky annulus with inner and outer radii of $48^{\prime \prime}$ and $80^{\prime \prime}$, respectively. Visual inspection and correction factors suggested by the SSC were used in all cases. The resulting fluxes are given in Table 2, together with the other IRAC and MIPS fluxes from c2d.

\subsection{Optical Spectroscopy}

Optical spectra were obtained for 21 objects in our sample using the Wide Field Fibre Optical Spectrograph (WYFFOS; Bingham et al. 1994) on the $4.2 \mathrm{~m}$ William Herschel Telescope (WHT), the Intermediate dispersion Spectrograph and 


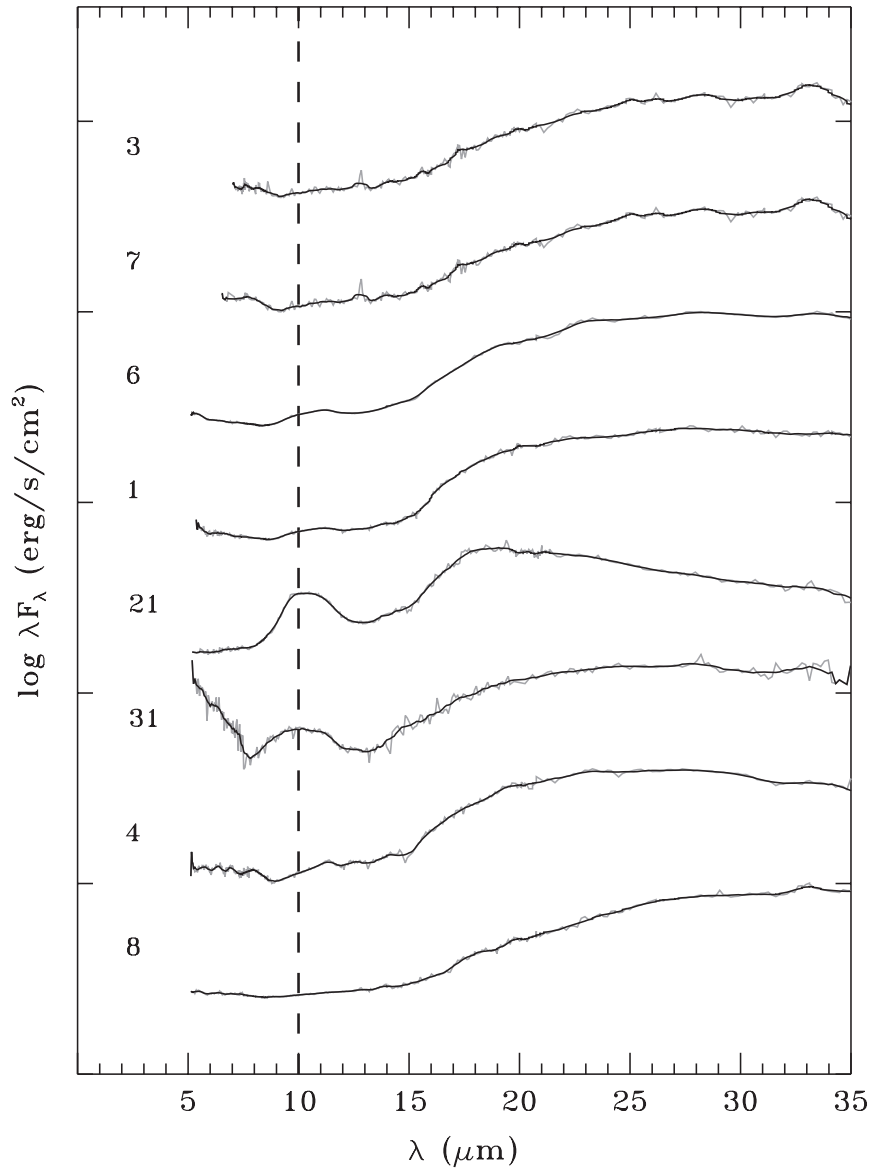

Figure 1. IRS spectra of the close to edge-on systems ordered from bottom to top with increasing effective temperature. The thick and gray lines are the binned and original spectra, respectively, and the numbers give their identifications in Table 1.

Imaging System (ISIS) at the WHT, the Intermediate Dispersion Spectrograph (IDS) at the Isaac Newton Telescope (INT), the Calar Alto Faint Object Spectrograph (CAFOS) at the $2.2 \mathrm{~m}$ Calar Alto Telescope, the Double Spectrograph (DBSP; Oke \& Gunn 1982) at the $5 \mathrm{~m}$ Hale Telescope at Palomar Observatory, and the R-C Spectrograph on the $1.5 \mathrm{~m}$ CTIO Telescope. These observations typically cover $\sim 5500-8500 \AA$ with a resolving power of $\sim 1000-5000$. Table 3 lists the instrument setups and observation $\log$.

Data were reduced and spectra were extracted using standard methods in IDL and IRAF. No telluric correction or flux calibration was performed. For WYFFOS, a fiber-fed multiobject spectrograph sky subtraction was obtained from fibers that were placed on blank sky regions (see Oliveira et al. 2009 for details).

\subsection{IRAM-30 m Observations}

A subsample of 17 of the northern targets was observed with the IRAM-30 m Telescope in Pico Veleta. The observations were carried out with MAMBO-2 (Kreysa et al. 2002) mounted on the IRAM-30 m Telescope during the 2007 winter and summer pool sessions. MAMBO-2 is a 117-bolometer array with a halfpower spectral bandwidth of $80 \mathrm{GHz}$ centered on $\sim 250 \mathrm{GHz}$ $(1.2 \mathrm{~mm})$, yielding a beam size of $11^{\prime \prime}$. The data were analyzed with the MOPSI software (Zylka 1998). The flux calibration was performed by observing either Mars or Uranus to determine the flux conversion factor. For each channel, the sky noise was

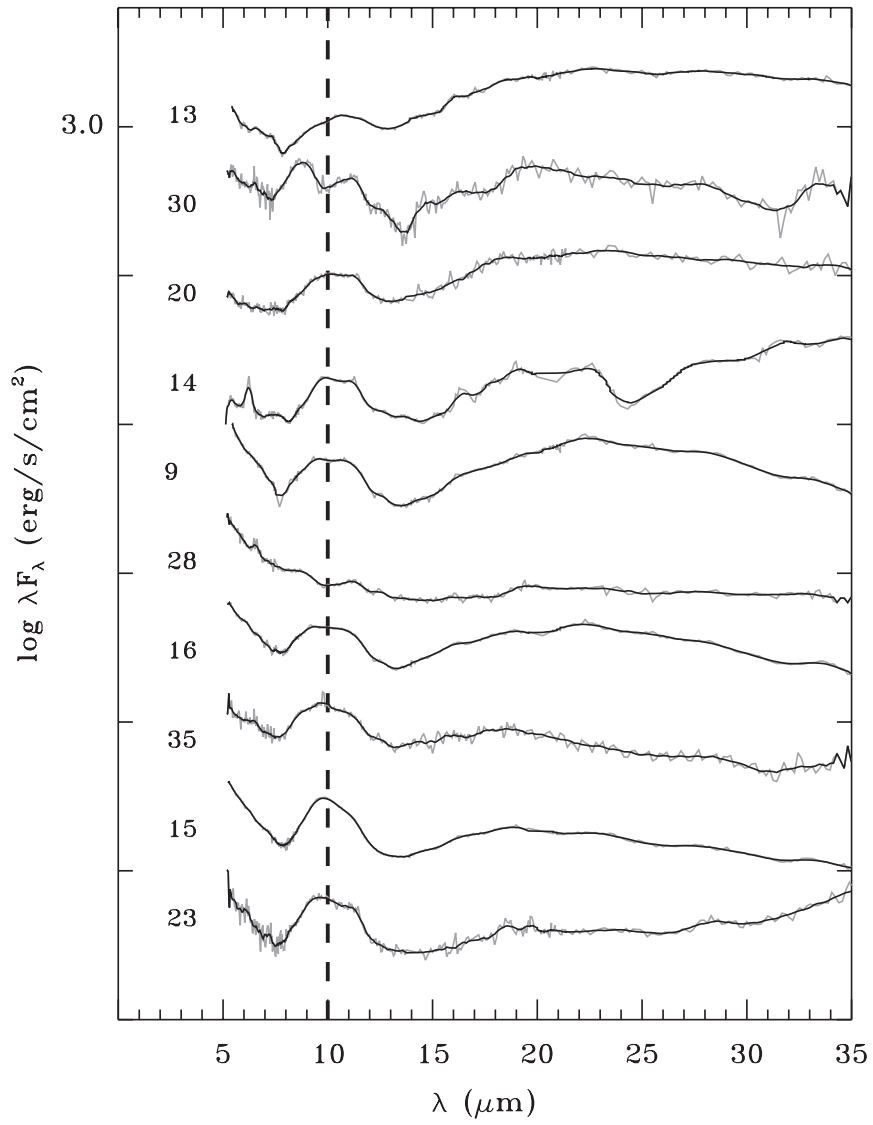

Figure 2. IRS spectra of the disks in the sample for which inner holes were not required to obtain a good SED fit, organized from bottom to top with an increasing SED slope $\alpha$. The thick and gray lines are the binned and original spectra, respectively, and the numbers give their identifications in Table 1.

subtracted by computing the weighted mean of the signals from the surrounding six channels.

All Perseus sources from our sample (see Table 1) were observed, except numbers $3,6,8$, and 11 . We also observed sources 20, 21, and 22 in Ophiuchus and sources 33, 34, and 35 in Serpens. The ON/OFF observing mode was used with a throw of $35^{\prime \prime}$ and each source was observed until a $>3 \sigma$ detection was obtained, or until an rms of $0.7 \mathrm{mJy}$ (whenever possible) was reached. The integration times ranged from 10 to 40 minutes. In general, the sky noise of the array was low ( $<80 \mathrm{mJy})$, and was never larger than $\sim 100 \mathrm{mJy}$. Ten sources were detected with fluxes between 1 and $10 \mathrm{mJy}$, while the other seven have upper limits between 1 and $7 \mathrm{mJy}$. These fluxes can be found in Column 8 of Table 2.

\section{STELLAR PROPERTIES}

Stellar properties must be determined to understand and interpret data on the surrounding disks. Optical spectra are used here to measure spectral types and to determine whether accretion is ongoing. We obtained optical spectra for 21 of the 35 objects in our sample. Of the other 14 stars, 11 have published spectral types and accretion properties. For the remaining three sources, spectral types are estimated from an SED analysis of broadband photometry, and accretion properties are left uncertain. The SED fits are also used to measure the extinction to each source. Results are presented in Table 4.

For the 21 objects for which we obtained optical spectra, spectral types of $\mathrm{G}$ and $\mathrm{K}$ stars were assigned by finding a 


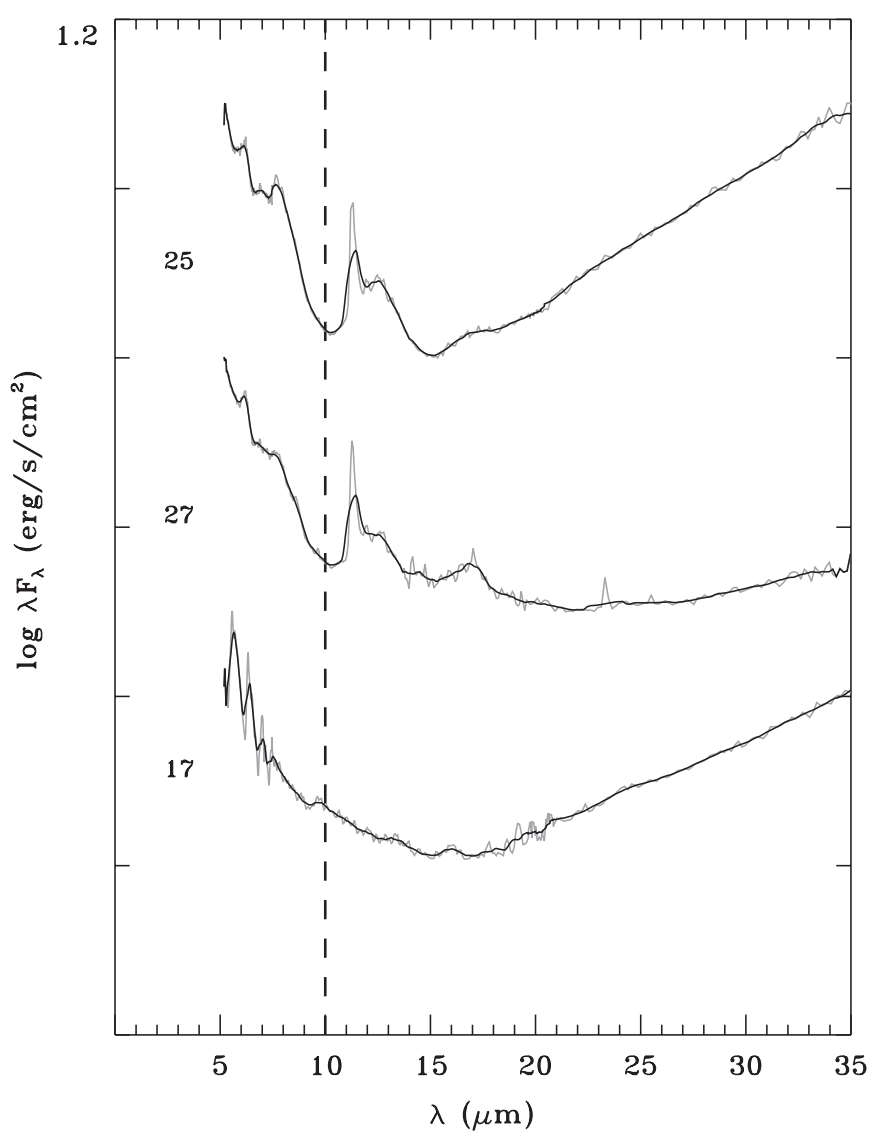

Figure 3. IRS spectra of the disks with potentially extended mid-IR emission from surrounding clouds ordered from bottom to top with increasing stellar mass. Object 17 (Sz 84) is shown here as a comparison due to its similar IRS spectrum, although it is classified as a cold disk. The thick and gray lines are the binned and original spectra, respectively, and the numbers give their identifications in Table 1.

best match to the depth of photospheric absorption features in template spectra obtained from the EXPORT spectral library (Mora et al. 2001). Spectral types of M-dwarfs were assigned by finding the best match to the $\mathrm{TiO}$ absorption bands from template spectra (Montes et al. 1997). The uncertainty in SpT is typically $\sim$ three subclasses for $\mathrm{G}$ and $\mathrm{K}$ stars and one subclass for $\mathrm{M}$ stars. Object 6 is classified as a continuum object because no spectral features were detected in the low-resolution spectrum. Any possible optical veiling was not considered for the other objects in the sample. A more detailed description of our spectral typing and the stellar properties of all the Serpens objects are presented by Oliveira et al. (2009). Literature values for SpT are adopted for several objects that we did not observe and for several objects where the literature $\mathrm{SpT}$ is more reliable than the SpT from our spectra. Spectral types are converted to $T_{\text {eff }}$ using the scales in Kenyon \& Hartmann (1995) for objects earlier than M0 and Luhman et al. (2003) for those later than or equal to M0, to account for the lower surface gravity atmospheres of mid M stars.

The extinction and luminosity for each object were calculated using SED fits to broadband optical and near-IR photometry (see Table 5 for photometry and Section 4.2 for a discussion on the assumed extinction law). Assumed distances are listed at the bottom of Table 4. NEXTGEN models of stellar photospheres (Hauschildt et al. 1999) are used as templates for the broadband emission. The Two Micron All Sky Survey (2MASS) and any optical photometry are the primary constraints on extinction and stellar luminosity. For the three objects that lack optical

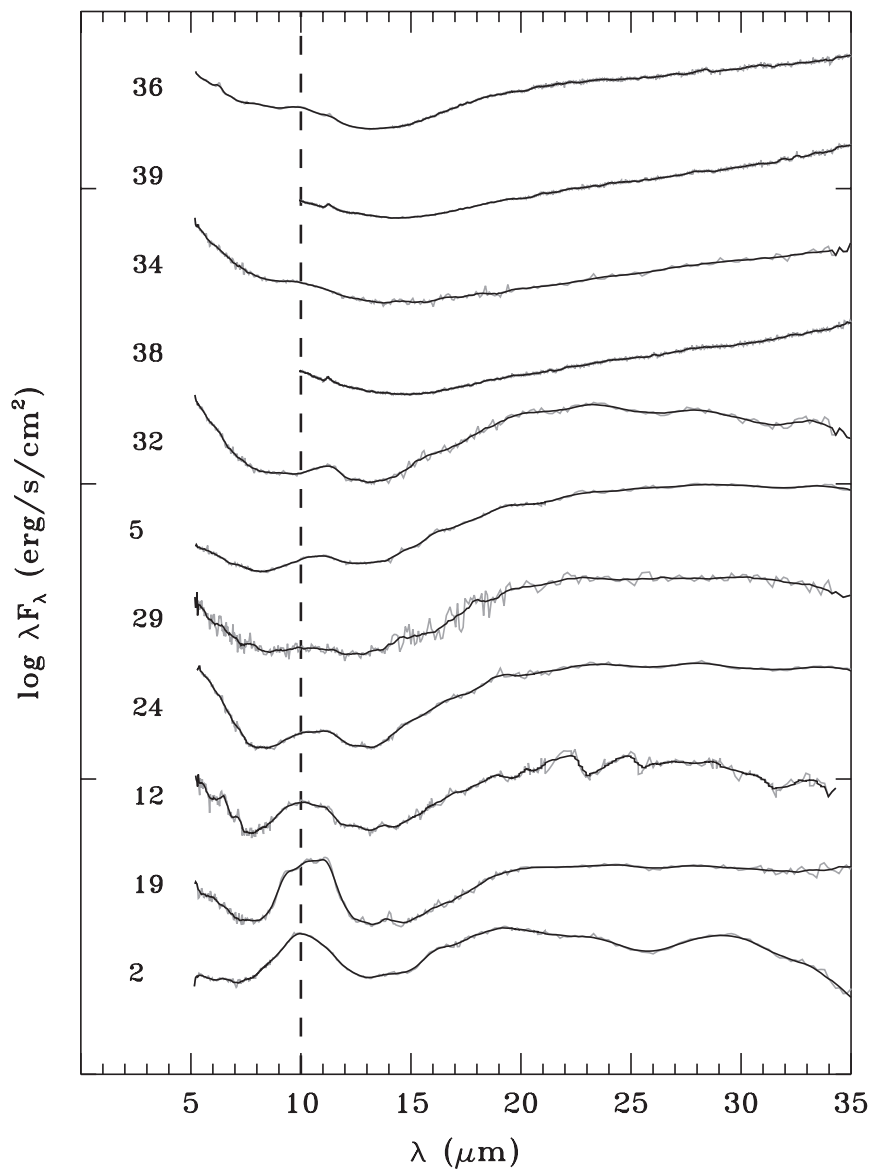

Figure 4. IRS spectra of the accreting cold disks in the sample ordered from bottom to top with increasing inner hole radii. The thick and gray lines are the binned and original spectra, respectively, and the numbers give their identifications in Table 1.

spectra (identified with a "2" in Table 4), the SED fits are also used to estimate SpT. However, these SpT estimates have large uncertainties because they are based on JHK colors, which are not very sensitive to spectral types from mid-K through $\mathrm{M}$ (Leggett 1992; Kenyon \& Hartmann 1995).

Stellar ages and masses are estimated by comparing the stellar luminosity and effective temperature to the pre-main-sequence tracks of Baraffe et al. (1998) for low-mass stars $\left(<1.4 M_{\odot}\right.$, mixing length $=1$ ) and of Siess et al. (2000) for highermass stars. Since uncertainties in stellar age are large, they are not tabulated here and we focus the analysis on the betterconstrained stellar masses.

\subsection{Edge-on Disks}

In the process of determining the stellar properties, it became clear that a fraction of the sample sources were actually edge-on disks. Inferring disk structure from an SED analysis is difficult when the disk is viewed edge-on and occults the star. In these cases, optical and near-IR light from the star and inner disk are only seen in reflected emission (e.g., Padgett et al. 1999). The strength of such emission depends on the precise viewing angle and the disk flaring, but is always much fainter than it would be if the disk were viewed without the large absorption column from the edge-on disk. Emission from outflows, seen prominently in optical forbidden lines, are not occulted by the disk and can therefore have large equivalent widths (White \& Hillenbrand 2004). 


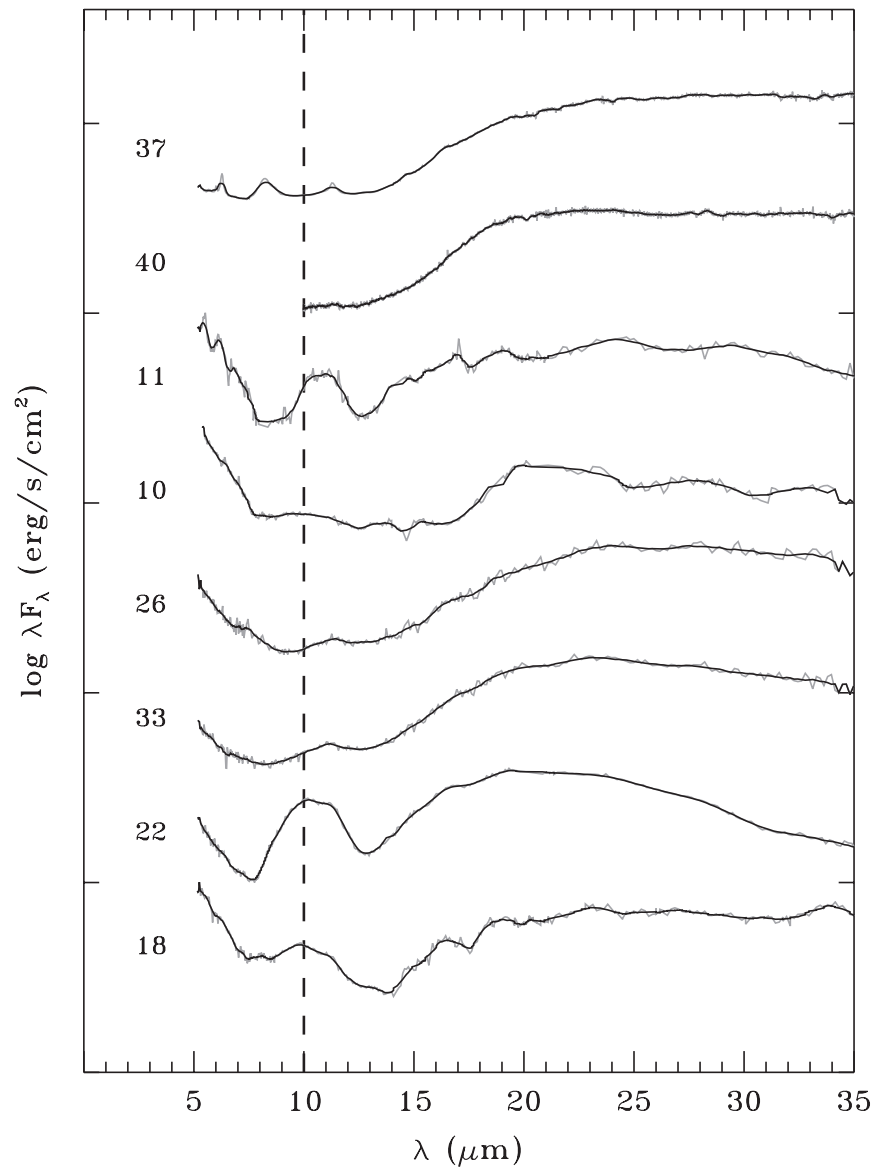

Figure 5. IRS spectra of the non-accreting or non-classified cold disks ordered from bottom to top with increasing inner hole radii. The thick and gray lines are the binned and original spectra, respectively, and the numbers give their identifications in Table 1.

Based on the existing data on our sources, we establish four criteria to determine whether a disk is viewed edge-on: (1) a low ratio of photospheric luminosity to main-sequence luminosity, assessed at $10^{8}$ Myr from Baraffe et al. (1998); (2) large equivalent widths in optical forbidden lines; (3) strong silicate and/or ice absorption in the IRS spectra; and (4) high infrared luminosity compared to stellar luminosity. Any one of these criteria can be met either by edge-on disks or by other phenomena, due to potential errors in distance and extinctions and the absence of accretion/outflow activity. However, the presence of at least two of these four criteria should establish in most cases that the disk is edge-on. Eight of the sources were selected as edge-on disks and the reasons are marked in Table 6. No further analysis was done on these disks as the stellar properties are too uncertain.

\subsection{Accretion Properties}

The presence or absence of accretion can be assessed from the strength and shape of emission in several optical lines, most prominently $\mathrm{H} \alpha$. Though some $\mathrm{H} \alpha$ emission is produced in chromospheres of late-type stars, accretion typically generates $\mathrm{H} \alpha$ emission with much larger equivalent widths and broader spectral profiles (see Hartmann 1998 for a comprehensive description of accretion phenomenology and diagnostics). A $10 \AA$ cutoff in equivalent width has historically been used to identify accretion, though this rough cutoff depends on spectral type (White \& Basri 2003; Fang et al. 2009). More recently, the $\mathrm{H} \alpha 10 \%$ width (defined as the full width of the $\mathrm{H} \alpha$ line at $10 \%$ of the peak flux) has been used to discriminate between accretors and non-accretors (White \& Basri 2003; Muzerolle et al. 2003; Jayawardhana et al. 2006). Accretion/outflow activity can also produce asymmetries and absorption components within the line profile (e.g., Reipurth et al. 1996; Muzerolle et al. 2003; Kurosawa et al. 2006) and variable line fluxes and shapes (e.g., Nguyen et al. 2009). For any of the criteria, a small accretion rate relative to the stellar luminosity is not detectable.

To classify the accretion properties of stars in our optical sample, a Gaussian profile is fit to the $\mathrm{H} \alpha$ line. We then measure the equivalent width and, when possible, the $\mathrm{H} \alpha 10 \%$ width from the Gaussian profile, after accounting for the resolution of each observation (see a more complete discussion of this method in Oliveira et al. 2009). Uncertainties in the $\mathrm{H} \alpha 10 \%$ widths range from 50 to $150 \mathrm{~km} \mathrm{~s}^{-1}$. The $\mathrm{H} \alpha$ equivalent width and $10 \%$ width are then used together with the spectral type to assess whether accretion is ongoing, adhering to the loose guidelines suggested by White \& Basri (2003) and Fang et al. (2009). Typically, a star is classified as an accretor if the $\mathrm{H} \alpha$ $10 \%$ width is $>300 \mathrm{~km} \mathrm{~s}^{-1}$ and the $\mathrm{H} \alpha$ equivalent width is $>3 \AA$ for an early-K star, $>10 \AA$ for late-K star, and $>20 \AA$ for an $\mathrm{M}$ star.

We classify 21 stars in our sample as accretors and eight stars as non-accretors, with six cases lacking information. In many cases, an object is clearly accreting. As explained below, some classifications are ambiguous both because some accretors and non-accretors have similar $\mathrm{H} \alpha 10 \%$ and equivalent widths and because the uncertainties in $\mathrm{H} \alpha 10 \%$ width are large. Objects 23 and 24 have weak $\mathrm{H} \alpha$ equivalent widths but broad $\mathrm{H} \alpha$ line shapes. Such observations could be explained if accretion is present with strong absorption, either from outflows or the accretion flow itself, which suppresses the $\mathrm{H} \alpha$ emission (e.g., Reipurth et al. 1996), if the star is an unresolved binary with only one accreting component, or if the stars are chromospherically active with a large $v \sin i$ (Jayawardhana et al. 2006). The highest-resolution spectrum of object 24 shows absorption in $\mathrm{H} \alpha$, while two of the three show [O I] in emission. This star is therefore classified as an accretor. On the other hand, object 23 shows no other evidence of accretion and is classified as a non-accretor. Object 18 is left unclassified because the $\mathrm{H} \alpha$ equivalent width is ambiguous between accretion and nonaccretion. The $\mathrm{H} \alpha$ emission from DoAr 21 (object 25) has varied on long timescales, but with no definitive evidence for accretion (Jensen et al. 2009). Temporal variations in $\mathrm{H} \alpha$ also introduce uncertainty in classifying the presence or absence of accretion. A mass-dependent bias likely exists in this classification scheme because the stronger photospheric continuum emission and spectral-type degeneracy for stars with early spectral types can mask $\mathrm{H} \alpha$ emission produced by weak-to-moderate accretion onto the star. Based on these uncertainties, we suspect that a small number of objects in our sample (estimated at $<10 \%$ ) might be misclassified as accretors or non-accretors.

To quantify accretion, we convert the $\mathrm{H} \alpha 10 \%$ widths to accretion rate using the relationship derived by Natta et al. (2004). Methodological differences in measuring the $\mathrm{H} \alpha 10 \%$ width between this work and Natta et al. (2004) likely leads to an order-of-magnitude uncertainty in the listed accretion rates. For late-K and early-M dwarfs, which comprise the bulk of our sample, the $\mathrm{H} \alpha 10 \%$ width is not sensitive to accretion rates $<10^{-10} M_{\odot} \mathrm{yr}^{-1}$. For earlier spectral types, the sensitivity worsens with larger accretion rates. Table 4 gives the $\mathrm{H} \alpha$ 
Table 2

IRAC, MIPS, and MAMBO $1.3 \mathrm{~mm}$ Fluxes for the Cold Disk Candidate Sample

\begin{tabular}{|c|c|c|c|c|c|c|c|}
\hline No. & $\begin{array}{c}\text { IRAC } 3.6 \\
(\mathrm{mJy})\end{array}$ & $\begin{array}{c}\text { IRAC } 4.5 \\
(\mathrm{mJy})\end{array}$ & $\begin{array}{c}\text { IRAC } 5.8 \\
(\mathrm{mJy})\end{array}$ & $\begin{array}{c}\text { IRAC } 8.0 \\
(\mathrm{mJy})\end{array}$ & $\begin{array}{c}\text { MIPS } 24.0 \\
(\mathrm{mJy})\end{array}$ & $\begin{array}{l}\text { MIPS 70.0 } \\
(\mathrm{mJy})\end{array}$ & $\begin{array}{c}1.3 \mathrm{~mm} \\
(\mathrm{mJy})\end{array}$ \\
\hline 1 & $1.45 \mathrm{e}+00 \pm 1.52 \mathrm{e}-02$ & $1.18 \mathrm{e}+00 \pm 1.52 \mathrm{e}-02$ & $9.55 \mathrm{e}-01 \pm 2.08 \mathrm{e}-02$ & $1.21 \mathrm{e}+00 \pm 2.19 \mathrm{e}-02$ & $4.25 \mathrm{e}+01 \pm 2.44 \mathrm{e}-01$ & $1.22 \mathrm{e}+02 \pm 4.69 \mathrm{e}+00$ & $<1.00 \mathrm{e}+00$ \\
\hline 2 & $1.68 \mathrm{e}+01 \pm 2.74 \mathrm{e}-01$ & $1.47 \mathrm{e}+01 \pm 2.74 \mathrm{e}-01$ & $1.21 \mathrm{e}+01 \pm 1.02 \mathrm{e}-01$ & $1.75 \mathrm{e}+01 \pm 1.20 \mathrm{e}-01$ & $1.78 \mathrm{e}+02 \pm 9.73 \mathrm{e}-01$ & $3.40 \mathrm{e}+02 \pm 9.35 \mathrm{e}+01$ & $3.70 \mathrm{e}+00 \pm 9.00 \mathrm{e}-01$ \\
\hline 3 & $9.64 \mathrm{e}-01 \pm 27.40 \mathrm{e}-03$ & $9.87 \mathrm{e}-01 \pm 7.40 \mathrm{e}-03$ & $8.28 \mathrm{e}-01 \pm 1.15 \mathrm{e}-02$ & $8.94 \mathrm{e}-01 \pm 1.34 \mathrm{e}-02$ & $1.14 \mathrm{e}+01 \pm 1.21 \mathrm{e}-01$ & $4.00 \mathrm{e}+01 \pm 4.06 \mathrm{e}+00$ & \\
\hline 4 & $2.01 \mathrm{e}+00 \pm 3.51 \mathrm{e}-02$ & $2.00 \mathrm{e}+00 \pm 3.51 \mathrm{e}-02$ & $2.05 \mathrm{e}+00 \pm 3.10 \mathrm{e}-02$ & $3.28 \mathrm{e}+00 \pm 3.21 \mathrm{e}-02$ & $6.78 \mathrm{e}+01 \pm 4.41 \mathrm{e}-01$ & $<3.75 \mathrm{e}+01$ & $1.20 \mathrm{e}+00 \pm 1.30 \mathrm{e}+00$ \\
\hline 5 & $1.28 \mathrm{e}+01 \pm 2.68 \mathrm{e}-01$ & $9.33 \mathrm{e}+00 \pm 2.68 \mathrm{e}-01$ & $6.96 \mathrm{e}+00 \pm 6.71 \mathrm{e}-02$ & $6.19 \mathrm{e}+00 \pm 4.73 \mathrm{e}-02$ & $9.55 \mathrm{e}+01 \pm 6.05 \mathrm{e}-01$ & $3.06 \mathrm{e}+02 \pm 1.31 \mathrm{e}+01$ & $6.30 \mathrm{e}+00 \pm 1.10 \mathrm{e}+00$ \\
\hline 6 & $1.51 \mathrm{e}+01 \pm 1.43 \mathrm{e}-01$ & $1.50 \mathrm{e}+01 \pm 1.43 \mathrm{e}-01$ & $1.05 \mathrm{e}+01 \pm 6.43 \mathrm{e}-02$ & $8.42 \mathrm{e}+00 \pm 5.92 \mathrm{e}-02$ & $4.31 \mathrm{e}+02 \pm 2.33 \mathrm{e}+00$ & $1.22 \mathrm{e}+03 \pm 8.20 \mathrm{e}+00$ & $<7.00 \mathrm{e}-01$ \\
\hline 7 & $1.46 \mathrm{e}+00 \pm 7.30 \mathrm{e}-02$ & $1.18 \mathrm{e}+00 \pm 5.86 \mathrm{e}-02$ & $1.02 \mathrm{e}+00 \pm 6.46 \mathrm{e}-02$ & $1.38 \mathrm{e}+00 \pm 7.64 \mathrm{e}-02$ & $4.17 \mathrm{e}+01 \pm 3.86 \mathrm{e}+00$ & $1.45 \mathrm{e}+02 \pm 4.06 \mathrm{e}+00$ & $1.30 \mathrm{e}+00 \pm 5.00 \mathrm{e}-01$ \\
\hline 8 & $1.05 \mathrm{e}+00 \pm 1.54 \mathrm{e}-02$ & $1.03 \mathrm{e}+00 \pm 1.54 \mathrm{e}-02$ & $8.18 \mathrm{e}-01 \pm 1.96 \mathrm{e}-02$ & $6.43 \mathrm{e}-01 \pm 1.81 \mathrm{e}-02$ & $5.75 \mathrm{e}+01 \pm 3.29 \mathrm{e}-01$ & $2.88 \mathrm{e}+02 \pm 6.25 \mathrm{e}+00$ & \\
\hline 9 & $9.37 \mathrm{e}+01 \pm 1.11 \mathrm{e}+00$ & $8.70 \mathrm{e}+01 \pm 1.11 \mathrm{e}+00$ & $7.81 \mathrm{e}+01 \pm 4.61 \mathrm{e}-01$ & $8.57 \mathrm{e}+01 \pm 7.27 \mathrm{e}-01$ & $3.07 \mathrm{e}+02 \pm 1.84 \mathrm{e}+00$ & $4.02 \mathrm{e}+02 \pm 8.60 \mathrm{e}+00$ & $1.30 \mathrm{e}+01 \pm 1.30 \mathrm{e}+00$ \\
\hline 10 & $1.32 \mathrm{e}+01 \pm 1.21 \mathrm{e}-01$ & $9.87 \mathrm{e}+00 \pm 1.21 \mathrm{e}-01$ & $7.23 e+00 \pm 5.75 e-02$ & $5.50 \mathrm{e}+00 \pm 4.83 \mathrm{e}-02$ & $4.68 \mathrm{e}+01 \pm 2.81 \mathrm{e}-01$ & $<8.39 \mathrm{e}+01$ & $<1.40 \mathrm{e}+00$ \\
\hline 11 & $6.68 \mathrm{e}+00 \pm 4.26 \mathrm{e}-02$ & $5.22 \mathrm{e}+00 \pm 4.26 \mathrm{e}-02$ & $4.21 \mathrm{e}+00 \pm 2.74 \mathrm{e}-02$ & $3.62 \mathrm{e}+00 \pm 4.19 \mathrm{e}-02$ & $1.39 \mathrm{e}+01 \pm 1.62 \mathrm{e}-01$ & $2.50 \mathrm{e}+01 \pm 6.60 \mathrm{e}+01$ & \\
\hline 12 & $1.13 \mathrm{e}+01 \pm 6.18 \mathrm{e}-02$ & $8.65 \mathrm{e}+00 \pm 6.18 \mathrm{e}-02$ & $6.47 \mathrm{e}+00 \pm 3.54 \mathrm{e}-02$ & $4.56 \mathrm{e}+00 \pm 3.94 \mathrm{e}-02$ & $2.10 \mathrm{e}+01 \pm 1.53 \mathrm{e}-01$ & $<7.60 \mathrm{e}+01$ & $<1.40 \mathrm{e}+00$ \\
\hline 13 & $1.23 \mathrm{e}+01 \pm 1.71 \mathrm{e}-01$ & $1.43 \mathrm{e}+01 \pm 1.71 \mathrm{e}-01$ & $1.31 \mathrm{e}+01 \pm 1.14 \mathrm{e}-01$ & $1.41 \mathrm{e}+01 \pm 1.40 \mathrm{e}-01$ & $1.23 \mathrm{e}+02 \pm 8.81 \mathrm{e}-01$ & $<1.37 \mathrm{e}+02$ & $1.16 \mathrm{e}+01 \pm 1.00 \mathrm{e}+00$ \\
\hline 14 & $1.32 \mathrm{e}+01 \pm 2.18 \mathrm{e}-01$ & $1.15 \mathrm{e}+01 \pm 2.18 \mathrm{e}-01$ & $8.93 \mathrm{e}+00 \pm 1.04 \mathrm{e}-01$ & $1.15 \mathrm{e}+01 \pm 2.06 \mathrm{e}-01$ & $6.71 \mathrm{e}+01 \pm 5.85 \mathrm{e}-01$ & $<2.16 \mathrm{e}+03$ & $<1.46 \mathrm{e}+00$ \\
\hline 15 & $1.27 \mathrm{e}+02 \pm 1.62 \mathrm{e}+00$ & $1.02 \mathrm{e}+02 \pm 1.62 \mathrm{e}+00$ & $7.66 \mathrm{e}+01 \pm 4.10 \mathrm{e}-01$ & $7.07 \mathrm{e}+01 \pm 5.18 \mathrm{e}-01$ & $1.75 \mathrm{e}+02 \pm 8.47 \mathrm{e}-01$ & $1.31 \mathrm{e}+02 \pm 7.86 \mathrm{e}+00$ & $<1.20 \mathrm{e}+00$ \\
\hline 16 & $1.16 \mathrm{e}+02 \pm 6.70 \mathrm{e}+00$ & $8.65 \mathrm{e}+01 \pm 4.69 \mathrm{e}+00$ & $7.71 \mathrm{e}+01 \pm 3.99 \mathrm{e}+00$ & $9.68 \mathrm{e}+01 \pm 4.78 \mathrm{e}+00$ & $2.48 \mathrm{e}+02 \pm 2.30 \mathrm{e}+01$ & $3.18 \mathrm{e}+02 \pm 3.37 \mathrm{e}+00$ & \\
\hline 17 & $4.20 \mathrm{e}+01 \pm 3.51 \mathrm{e}-02$ & $2.90 \mathrm{e}+01 \pm 3.51 \mathrm{e}-02$ & $2.01 \mathrm{e}+01 \pm 3.10 \mathrm{e}-02$ & $1.20 \mathrm{e}+01 \pm 3.21 \mathrm{e}-02$ & $2.09 \mathrm{e}+01 \pm 4.41 \mathrm{e}-01$ & $2.44 \mathrm{e}+02 \pm 7.32 \mathrm{e}+01$ & $<3.60 \mathrm{e}+01$ \\
\hline 18 & $1.91 \mathrm{e}+01 \pm 9.30 \mathrm{e}-01$ & $1.42 \mathrm{e}+01 \pm 9.30 \mathrm{e}-01$ & $1.15 \mathrm{e}+01 \pm 5.60 \mathrm{e}-01$ & $1.09 \mathrm{e}+01 \pm 5.30 \mathrm{e}-01$ & $3.37 \mathrm{e}+01 \pm 3.18 \mathrm{e}+00$ & $1.10 \mathrm{e}+02 \pm 1.80 \mathrm{e}+01$ & $\ldots$ \\
\hline 19 & $1.14 \mathrm{e}+02 \pm 9.30 \mathrm{e}-01$ & $8.50 \mathrm{e}+01 \pm 9.30 \mathrm{e}-01$ & $6.10 \mathrm{e}+01 \pm 5.60 \mathrm{e}-01$ & $6.60 \mathrm{e}+01 \pm 5.30 \mathrm{e}-01$ & $2.71 \mathrm{e}+02 \pm 3.18 \mathrm{e}+00$ & $7.27 \mathrm{e}+02 \pm 1.80 \mathrm{e}+01$ & $\cdots$ \\
\hline 20 & $1.24 \mathrm{e}+01 \pm 6.00 \mathrm{e}-01$ & $1.20 \mathrm{e}+01 \pm 5.70 \mathrm{e}-01$ & $1.08 \mathrm{e}+01 \pm 5.20 \mathrm{e}-01$ & $1.52 \mathrm{e}+01 \pm 7.30 \mathrm{e}-01$ & $7.99 \mathrm{e}+01 \pm 7.39 \mathrm{e}+00$ & $<6.82 \mathrm{e}+01$ & $<4.60 \mathrm{e}+00$ \\
\hline 21 & $1.61 \mathrm{e}+00 \pm 8.00 \mathrm{e}-02$ & $1.60 \mathrm{e}+00 \pm 7.00 \mathrm{e}-02$ & $1.78 \mathrm{e}+00 \pm 1.00 \mathrm{e}-01$ & $7.72 \mathrm{e}+00 \pm 3.80 \mathrm{e}-01$ & $1.17 \mathrm{e}+02 \pm 1.08 \mathrm{e}+01$ & $<5.38 \mathrm{e}+02$ & $<4.40 \mathrm{e}+00$ \\
\hline 22 & $9.21 \mathrm{e}+01 \pm 4.58 \mathrm{e}+00$ & $6.15 \mathrm{e}+01 \pm 3.04 \mathrm{e}+00$ & $4.47 \mathrm{e}+01 \pm 2.14 \mathrm{e}+00$ & $5.13 \mathrm{e}+01 \pm 2.47 \mathrm{e}+00$ & $3.45 \mathrm{e}+02 \pm 3.20 \mathrm{e}+01$ & $<8.31 \mathrm{e}+01$ & $<5.40 \mathrm{e}+00$ \\
\hline 23 & $3.23 \mathrm{e}+01 \pm 1.61 \mathrm{e}+00$ & $2.44 \mathrm{e}+01 \pm 1.17 \mathrm{e}+00$ & $1.92 \mathrm{e}+01 \pm 9.20 \mathrm{e}-01$ & $2.23 \mathrm{e}+01 \pm 1.07 \mathrm{e}+00$ & $4.79 \mathrm{e}+01 \pm 4.47 \mathrm{e}+00$ & $<4.32 \mathrm{e}+02$ & $\ldots$ \\
\hline 24 & $6.07 \mathrm{e}+01 \pm 2.74 \mathrm{e}-01$ & $4.17 \mathrm{e}+01 \pm 2.74 \mathrm{e}-01$ & $3.09 \mathrm{e}+01 \pm 1.02 \mathrm{e}-01$ & $2.28 \mathrm{e}+01 \pm 1.20 \mathrm{e}-01$ & $1.59 \mathrm{e}+02 \pm 9.73 \mathrm{e}-01$ & $5.37 \mathrm{e}+02 \pm 7.88 \mathrm{e}+01$ & $\ldots$ \\
\hline 25 & $1.19 \mathrm{e}+03 \pm 1.94 \mathrm{e}+01$ & $8.45 \mathrm{e}+02 \pm 1.49 \mathrm{e}+01$ & $7.30 \mathrm{e}+02 \pm 1.00 \mathrm{e}+01$ & $6.39 \mathrm{e}+02 \pm 8.50 \mathrm{e}+00$ & $1.34 \mathrm{e}+03 \pm 3.42 \mathrm{e}+01$ & $1.20 \mathrm{e}+04 \pm 1.26 \mathrm{e}+03$ & $\ldots$ \\
\hline 26 & $9.80 \mathrm{e}+00 \pm 5.75 \mathrm{e}-01$ & $8.51 \mathrm{e}+00 \pm 5.75 \mathrm{e}-01$ & $7.03 \mathrm{e}+00 \pm 3.82 \mathrm{e}-01$ & $5.46 \mathrm{e}+00 \pm 2.98 \mathrm{e}-01$ & $4.34 \mathrm{e}+01 \pm 4.03 \mathrm{e}+00$ & $<2.87 \mathrm{e}+02^{\mathrm{a}}$ & $2.40 \mathrm{e}+01 \pm 6.00 \mathrm{e}-01$ \\
\hline 27 & $5.25 \mathrm{e}+01 \pm 2.97 \mathrm{e}+00$ & $3.67 \mathrm{e}+01 \pm 2.97 \mathrm{e}+00$ & $3.12 \mathrm{e}+01 \pm 1.68 \mathrm{e}+00$ & $2.85 \mathrm{e}+01 \pm 1.68 \mathrm{e}+00$ & $9.74 \mathrm{e}+00 \pm 9.20 \mathrm{e}-01$ & $1.17 \mathrm{e}+03 \pm 1.01 \mathrm{e}+02$ & $\ldots$ \\
\hline 28 & $2.13 \mathrm{e}+01 \pm 1.43 \mathrm{e}+00$ & $2.93 \mathrm{e}+01 \pm 1.43 \mathrm{e}+00$ & $2.25 \mathrm{e}+01 \pm 1.29 \mathrm{e}+00$ & $2.27 \mathrm{e}+01 \pm 1.10 \mathrm{e}+00$ & $4.02 \mathrm{e}+01 \pm 3.72 \mathrm{e}+00$ & $<2.87 \mathrm{e}+02^{\mathrm{a}}$ & $\ldots$ \\
\hline 29 & $4.46 \mathrm{e}+00 \pm 2.73 \mathrm{e}-01$ & $3.36 \mathrm{e}+00 \pm 2.73 \mathrm{e}-01$ & $2.39 \mathrm{e}+00 \pm 1.47 \mathrm{e}-01$ & $1.72 \mathrm{e}+00 \pm 1.03 \mathrm{e}-01$ & $1.60 \mathrm{e}+01 \pm 1.49 \mathrm{e}+00$ & $<2.87 \mathrm{e}+02^{\mathrm{a}}$ & $\ldots$ \\
\hline 30 & $1.36 \mathrm{e}+01 \pm 8.67 \mathrm{e}-01$ & $1.62 \mathrm{e}+01 \pm 8.67 \mathrm{e}-01$ & $1.51 \mathrm{e}+01 \pm 8.86 \mathrm{e}-01$ & $2.56 \mathrm{e}+01 \pm 1.26 \mathrm{e}+00$ & $6.05 \mathrm{e}+01 \pm 5.59 \mathrm{e}+00$ & $<5.00 \mathrm{e}+01$ & $\ldots$ \\
\hline 31 & $6.42 \mathrm{e}+00 \pm 4.61 \mathrm{e}-01$ & $7.33 \mathrm{e}+00 \pm 4.61 \mathrm{e}-01$ & $5.99 \mathrm{e}+00 \pm 3.46 \mathrm{e}-01$ & $8.05 \mathrm{e}+00 \pm 3.94 \mathrm{e}-01$ & $3.25 \mathrm{e}+01 \pm 3.01 \mathrm{e}+00$ & $1.35 \mathrm{e}+02 \pm 1.57 \mathrm{e}+01$ & $\ldots$ \\
\hline 32 & $3.05 \mathrm{e}+01 \pm 2.00 \mathrm{e}+00$ & $2.41 \mathrm{e}+01 \pm 2.00 \mathrm{e}+00$ & $1.70 \mathrm{e}+01 \pm 9.68 \mathrm{e}-01$ & $1.28 \mathrm{e}+01 \pm 6.61 \mathrm{e}-01$ & $7.42 \mathrm{e}+01 \pm 6.86 \mathrm{e}+00$ & $5.76 \mathrm{e}+01 \pm 1.31 \mathrm{e}+01$ & $\ldots$ \\
\hline 33 & $2.11 \mathrm{e}+01 \pm 1.24 \mathrm{e}+00$ & $1.99 \mathrm{e}+01 \pm 1.24 \mathrm{e}+00$ & $1.56 \mathrm{e}+01 \pm 8.31 \mathrm{e}-01$ & $1.28 \mathrm{e}+01 \pm 6.19 \mathrm{e}-01$ & $8.11 \mathrm{e}+01 \pm 7.49 \mathrm{e}+00$ & $1.26 \mathrm{e}+02 \pm 1.74 \mathrm{e}+01$ & $2.30 \mathrm{e}+00 \pm 6.00 \mathrm{e}-01$ \\
\hline 34 & $2.08 \mathrm{e}+01 \pm 1.19 \mathrm{e}+00$ & $1.37 \mathrm{e}+01 \pm 1.19 \mathrm{e}+00$ & $1.10 \mathrm{e}+01 \pm 5.91 \mathrm{e}-01$ & $6.97 \mathrm{e}+00 \pm 3.45 \mathrm{e}-01$ & $1.97 \mathrm{e}+01 \pm 1.83 \mathrm{e}+00$ & $1.40 \mathrm{e}+02 \pm 1.71 \mathrm{e}+01$ & $6.30 \mathrm{e}+00 \pm 6.00 \mathrm{e}-01$ \\
\hline 35 & $1.04 \mathrm{e}+01 \pm 6.24 \mathrm{e}-01$ & $9.28 \mathrm{e}+00 \pm 6.24 \mathrm{e}-01$ & $8.20 \mathrm{e}+00 \pm 4.53 \mathrm{e}-01$ & $1.06 \mathrm{e}+01 \pm 5.43 \mathrm{e}-01$ & $1.82 \mathrm{e}+01 \pm 1.69 \mathrm{e}+00$ & $1.76 \mathrm{e}+01 \pm 6.81 \mathrm{e}+00$ & $2.20 \mathrm{e}+00 \pm 9.00 \mathrm{e}-01$ \\
\hline
\end{tabular}

Note. ${ }^{\text {a }}$ Upper limits are estimates from nearby sources due to difficulties with the local background flux. 
Table 3

Optical Spectroscopy Observation Log

\begin{tabular}{|c|c|c|c|c|c|}
\hline Date & $\begin{array}{l}\text { Telescope }+ \\
\text { Instrument }\end{array}$ & Grism & $\begin{array}{c}\text { Wavelength } \\
\text { Coverage }(\AA)\end{array}$ & $\begin{array}{c}\text { Dispersion } \\
(\AA / \text { resel })\end{array}$ & ColheadID $^{\mathrm{a}}$ \\
\hline 2006 Nov 22 & WHT+WYFFOS & R600R & $5500-8500$ & 4 & $\begin{array}{c}2,3,(4), 5,7,(10),(11) \\
12,13,14,15 \\
\end{array}$ \\
\hline $\begin{array}{l}2006 \text { Dec } 13 \\
2007 \text { Jul } 26 \\
2008 \text { Jul } 22\end{array}$ & WHT+ISIS & R600R & $6000-8000$ & & $\begin{array}{c}1,(9) \\
24 \\
2\end{array}$ \\
\hline 2007 May 6 & INT+IDS & R600R & $4700-8700$ & & 22,24 \\
\hline 2008 Jun $23-25$ & $2.2 \mathrm{~m} / \mathrm{CAFOS}$ & R100 & $6000-9000$ & 2 & $21,23,24,25,29$ \\
\hline $\begin{array}{l}2008 \text { Dec } 30 \\
2009 \text { Jul } 21 \\
\end{array}$ & $\begin{array}{l}\text { Palomar/DBSP } \\
\text { Palomar/DBSP }\end{array}$ & $\begin{array}{l}\mathrm{R} 316 \\
\mathrm{R} 316 \\
\end{array}$ & $\begin{array}{l}6200-8700 \\
6200-8700 \\
\end{array}$ & 10 & $\begin{array}{c}4,6,8 \\
9,10 \\
\end{array}$ \\
\hline 2009 Oct 30 & WHT+ISIS & $\mathrm{R} 316$ & $5500-8300$ & & 10,13 \\
\hline 2009 Aug 3 & CTIO 1.5 m/R-C Spec & $47 / \mathrm{Ib}$ & $5630-6930$ & & 18,19 \\
\hline
\end{tabular}

Note.

${ }^{\mathrm{a}}()=$ low $\mathrm{S} / \mathrm{N}$.

equivalent widths, $10 \%$ widths, and mass accretion rates derived from this work, and Table 8 gives the final classification of each source.

\section{RESULTS: DISK AND DISK HOLE PARAMETERS}

\subsection{Disk Mass}

Millimeter continuum fluxes can be used to calculate disk masses assuming that the millimeter emission is optically thin and a gas-to-dust mass ratio of 100 . The disk masses can then be used in turn to constrain SED models. The disk masses, $M_{\text {Disk }}$, are calculated following Beckwith et al. (1990) and with standard assumptions and parameters. In the Rayleigh-Jeans limit,

$$
M_{\text {Disk }}=F_{v} \frac{d^{2}}{2 k\langle T\rangle} \frac{c^{2}}{v^{2} \kappa_{v}},
$$

where $F_{v}$ is the flux at frequency $v$, here $230 \mathrm{GHz}, d$ is the distance to the star (see Table 4), $k$ is the Boltzmann constant, and $\kappa_{\nu}$ is the opacity. It is assumed that the emission arises primarily from an approximately isothermal region with temperature $\langle T\rangle$. Average disk temperatures in the outer disk range from 10 to $50 \mathrm{~K}$ and here we have used $30 \mathrm{~K}$. One of the major uncertainties is the opacity $\kappa_{v}$. We follow Beckwith et al. (1990) and adopt $\kappa_{0}=0.02 \mathrm{~cm}^{2} \mathrm{~g}^{-1}$ anchored at $v_{0}=230 \mathrm{GHz}$, the frequency of our observations.

Disk masses are listed in Table 7 for the ten detected sources and the seven with upper limits. The majority $(80 \%)$ of the disks in our sample have disk masses less than $2 \times 10^{-3} M_{\odot}$ and the sample has an average $M_{\text {Disk }}$ of $4.5_{-1.4}^{+5.5} \times 10^{-3} M_{\odot}$. These are relatively low-mass disks compared to many millimeter studies, such as the large sample of T Tauri disks in Andrews \& Williams (2007) which have an average mass of $10^{-2} M_{\odot}$ using the same assumptions.

\subsection{Disk Hole Parameters from SED Modeling}

In order to determine the presence or absence of a hole, the full SED is needed to determine the disk structure. The SEDs for the stars in the sample are organized by type with edge-on disks (Figure 6), disks with holes (Figures 7 and 8), and disks without holes (Figure 9). The solid dots are dereddened fluxes, which contain the 2MASS JHK fluxes, the four IRAC fluxes from 3.6 to $8 \mu \mathrm{m}$, and the MIPS fluxes at 24 and $70 \mu \mathrm{m}$ for all sources, plus optical or $1.3 \mathrm{~mm}$ fluxes for a smaller subsample of the objects. In some cases, $70 \mu \mathrm{m}$ photometry produced only upper limits, often accompanied by non-detections at $1.3 \mathrm{~mm}$, and thus severely limits information about the outer disk. The solid dots are the dereddened fluxes, the solid line is the IRS spectrum binned at a resolution of $1 \mu \mathrm{m}$, and the gray dotted line is the stellar Kurucz model of the central star. The differences between the Kurucz and the NEXTGEN stellar models used above are negligible for the purposes of disk characterization.

The data were dereddened using the $A_{\mathrm{V}}$ values listed in Table 4 and the extinction law by Weingartner \& Draine (2001) with $R_{V}=5.5$. Recently, Evans et al. (2009) and Chapman et al. (2009) have suggested that this larger value of $R_{V}$ is more suitable for star-forming clouds than the typical value of $R_{V}=3.1$, which is based on models and observations of the diffuse interstellar medium (ISM). The higher $R_{V}$ value reflects larger maximum dust grain sizes and results in stronger dereddening effects throughout the mid-IR. Dereddened fluxes are two to four times higher at $24 \mu \mathrm{m}$ and $A_{V}>10$ than with $R_{V}=3.1$. However, observational measures of extinction indicate that the large amount of reddening is in agreement with observations and may even underestimate the actual effect in star-forming regions (Chapman et al. 2009; Flaherty et al. 2007).

As the extinction law is not flat throughout the mid-IR, errors in the mid-IR extinction law could potentially artificially create or remove SED deficits, mimicking inner holes. Corrections are much smaller at low $A_{V}$, so effects only become significant for $A_{V}>10$. Based on observations, the extinction law used here is conservative, with some cold disks potentially being missed due to inadequate dereddening around $20 \mu \mathrm{m}$. With such large grain sizes in the absorbing dust, silicate grain opacities are expected to have strong effects on the $10 \mu \mathrm{m}$ silicate feature. In a few dereddened SEDs, strong silicate features, which are not present in the uncorrected spectra, are seen. The effects of extinction on the silicate features are complex and dependent on extinction and line-of-sight ice and dust composition, which may not be uniform throughout star-forming regions (McClure 2009). Due to this uncertainty, all work on the silicate feature in this paper 
Table 4

Stellar Parameters for the Stars in the Sample

\begin{tabular}{|c|c|c|c|c|c|c|c|c|c|c|}
\hline No. & Object ID & SpT & Ref & $\begin{array}{c}A_{V} \\
(\mathrm{mag})\end{array}$ & $\begin{array}{l}T_{\text {eff }} \\
(\mathrm{K}) \\
\end{array}$ & $\begin{array}{c}L_{*} \\
\left(L_{\odot}\right)\end{array}$ & Mass & $\begin{array}{c}\mathrm{EW}[\mathrm{H} \alpha]^{\mathrm{a}} \\
(\AA)\end{array}$ & $\begin{array}{l}\mathrm{H} \alpha[10 \%] \\
\left(\mathrm{km} \mathrm{s}^{-1}\right)\end{array}$ & $\begin{array}{c}\log \dot{M_{\mathrm{acc}}} \\
\left(M_{\odot} \mathrm{yr}^{-1}\right) \\
\end{array}$ \\
\hline 2 & ASR 118 & K4 & 1 & 13.0 & 4590 & 1.17 & 1.36 & 26 & 293 & -10.2 \\
\hline 5 & SSTc2d J032929.3+311835 & M0 & 1 & $\ldots$ & 3850 & 0.09 & $\ldots$ & 4.8 & $\ldots$ & $\ldots$ \\
\hline 10 & SSTc2d J034227.1+314433 & K7 & 1 & 5.0 & 4060 & 0.22 & 0.82 & 4.3 & $\cdots$ & $\cdots$ \\
\hline 11 & $\mathrm{Cl}^{*}$ IC 348 LRL 190 & M3.75 & 6 & 6.5 & 3306 & 0.09 & 0.35 & 5 & $\ldots$ & $\ldots$ \\
\hline 12 & SSTc2d J034434.8+315655 & M3 & 1 & 3.0 & 3415 & 0.12 & 0.45 & 130 & 504 & -8.0 \\
\hline 17 & Sz 84 & M5.5 & 3 & 0.5 & 3057 & 0.15 & 0.14 & 44 & $\ldots$ & $\ldots$ \\
\hline 18 & SSTc2d J161029.6-392215 & M5 & 1 & 1.0 & 3125 & 0.08 & 0.21 & 18 & $\ldots$ & $\ldots$ \\
\hline 19 & RX J1615.3-3255 & K4 & 1 & 1.0 & 4590 & 0.85 & 1.28 & 26 & $\ldots$ & $\ldots$ \\
\hline 22 & SSTc2d J162245.4-243124 & M2 & 1 & 2.0 & 3580 & 0.29 & 0.59 & 5.0 & 224 & $\ldots$ \\
\hline 24 & SSTc2d J162506.9-235050 & M0.5 & 1 & 3.0 & 3778 & 0.21 & 0.77 & 4.6 & 505 & -8.0 \\
\hline 26 & SSTc2d J182829.1+002756 & K7 & 2 & 13.0 & 4060 & 0.34 & 0.94 & $\ldots$ & $\ldots$ & $\ldots$ \\
\hline 29 & SSTc2d J182911.5+002039 & M0 & 7 & 6.0 & 3850 & 0.13 & 0.72 & 25 & 656 & -6.5 \\
\hline 32 & SSTc2d J182935.6+003504 & K7 & 7 & 4.0 & 4060 & 0.52 & 1.06 & 10.9 & 273 & -10.2 \\
\hline 33 & SSTc2d J182936.2+004217 & F9 & 7 & 11.0 & 6115 & 1.89 & 1.30 & $\ldots$ & $\ldots$ & $\ldots$ \\
\hline 34 & SSTc2d J182944.1+003356 & M0 & 7 & 5.0 & 3850 & 0.54 & 0.93 & 37 & 444 & -8.6 \\
\hline 1 & SSTc2d J032835.1+302010 & K4 & 1 & 0.0 & 4590 & 0.07 & $\ldots$ & 8.1 & 449 & -8.5 \\
\hline 3 & SSTc2d J032903.9+305630 & G7 & 1 & 6.0 & 5630 & 0.05 & $\ldots$ & 54 & 344 & -9.6 \\
\hline 4 & SSTc2d J032924.1+311958 b & K5 & 1 & 5.0 & 4350 & 0.41 & 0.96 & 6.5 & 262 & -10.4 \\
\hline 6 & SSTc2d J033027.2+302830 b,c & $c^{d}$ & 1 & $\ldots$ & $\ldots$ & $\ldots$ & $\ldots$ & 106 & $\ldots$ & $\ldots$ \\
\hline 7 & SSTc2d J033035.5+311559b & K5 & 1 & $\ldots$ & 4350 & 0.07 & $\ldots$ & 15.1 & 405 & -9.0 \\
\hline 8 & SSTc2d J033038.2+303212b & $\mathrm{MO}^{\mathrm{e}}$ & 1 & $\ldots$ & 3850 & 0.05 & $\ldots$ & $\ldots$ & 194 & $\ldots$ \\
\hline 9 & LZK 21 & M0 & 1 & 6.0 & 3850 & 1.46 & 1.00 & 72 & $\ldots$ & $\ldots$ \\
\hline 13 & Cl* IC 348 LRL 265 & M3 & 1 & 17. & 3415 & 0.42 & 0.52 & 113 & $\ldots$ & $\ldots$ \\
\hline 14 & Cl* IC 348 LRL 110 & M0.5 & 1 & 5.0 & 3778 & 0.22 & 0.78 & 36 & 424 & -8.8 \\
\hline 15 & $\mathrm{LkH} \alpha 329$ & K5 & 1 & 5.0 & 4350 & 2.89 & 1.63 & 19.4 & 700 & -6.1 \\
\hline 16 & Hn 24 & M0.5 & 1 & 2.0 & 3778 & 0.75 & 0.92 & 0.2 & 311 & $\ldots$ \\
\hline 20 & SSTc2d J162148.5-234027 & M5.5 & 5 & 7.0 & 3057 & 0.04 & 0.14 & $\ldots^{\mathrm{f}}$ & $\cdots$ & $\cdots$ \\
\hline 21 & SSTc2d J162221.1-230403 ${ }^{b}$ & K5 & 1 & $\ldots$ & 4350 & 0.02 & $\ldots$ & 45.8 & 594 & -7.1 \\
\hline 23 & SSTc2d J162332.9-225847 & M0.5 & 1 & 5.0 & 3778 & 0.15 & 0.73 & 2.8 & 413 & $\ldots$ \\
\hline 25 & DoAr 21 & K1 & 4 & 6.0 & 5080 & 9.84 & 1.80 & $1-2$ & 450 & $\ldots$ \\
\hline 27 & SSTc2d J182858.1+001724 & G3 & 7 & 6.0 & 5830 & 4.76 & 1.4 & -2.2 & $\cdots$ & $\cdots$ \\
\hline 28 & SSTc2d J182907.0+003838 & K7 & 2 & 16.0 & 4060 & 0.6 & 1.04 & $\cdots$ & $\ldots$ & $\cdots$ \\
\hline 30 & SSTc2d J182915.6+003912 & K5 & 7 & 12.0 & 4350 & 0.45 & 1.00 & 22 & 532 & -7.7 \\
\hline 31 & SSTc2d J182915.6+003923 ${ }^{b}$ & K7 & 2 & 0.0 & 4060 & 0.20 & $\cdots$ & $\ldots$ & $\ldots$ & $\ldots$ \\
\hline 35 & SSTc2d J182947.3+003223 & M0 & 7 & 7.0 & 3850 & 0.28 & 0.85 & 49 & 618 & -6.9 \\
\hline
\end{tabular}

Notes. For clarity, cold disks are shown above the horizontal line while other objects are below. We used the c2d distances to the clouds to compute the stellar luminosities: 250 pc for Perseus, 187 pc for Cham. II, 150 pc for Lupus II and III, 120 pc for Ophiuchus, and 260 pc for Serpens.

${ }^{a} \mathrm{H} \alpha$ equivalent widths are listed as positive when seen in emission and negative when seen in absorption.

${ }^{\mathrm{b}}$ Objects identified as likely edge-on disks. Luminosities are presented to show that the stars are underluminous. In these cases, we do not calculate masses.

c Source is spatially extended in slit.

${ }^{\mathrm{d}}$ Continuum spectral type. The object is heavily veiled and no SpT was obtainable at low spectral resolution.

e Large uncertainty due to poor $\mathrm{S} / \mathrm{N}$ and large veiling.

f Accreting, based on the presence of Paschen $\beta$ emission (M. Gully-Santiago et al. 2010, in preparation).

References. (1) Spectral type from this work with optical spectroscopy; (2) Spectral type from this work with photometry; (3) Krautter et al. 1997; (4) Jensen et al. 2009; (5) M. Gully-Santiago et al. 2010, in preparation; (6) Luhman et al. 2003; (7) Oliveira et al. 2009.

has been done on spectra that have not been dereddened, with the caveat that sources with high $A_{V}$ have large errors (see also the discussion in Oliveira et al. 2010).

The two-dimensional radiative transfer model RADMC (Dullemond \& Dominik 2004), modified to include a density reduction simulating a hole, was used to model the SEDs. The model has a large number of input parameters and we have fixed the values of as many as possible. The model assumes a passive disk, which merely reprocesses the stellar radiation field and does not account for accretion luminosity and heating. This is justified since the disk to stellar luminosity ratios of the majority of the disks in this study are below 0.4 (see Table 7). Further discussion on the disk model dependency of the results can be found in Section 5.2. Stellar input parameters are mass, temperature, and luminosity, which were determined from optical spectroscopy and photospheric SED fits (see Table 4). A stellar Kurucz model is then used for the stellar radiation field. In terms of disk structure, the disk is assumed to be flared such that the surface height, $H$, varies with radius, $R$, as $H / R \propto R^{2 / 7}$, as in Chiang \& Goldreich (1997). The models do not calculate the hydrostatic equilibrium self-consistently, allowing the dust distribution to differ from the gas distribution (expected to be in hydrostatic equilibrium) due to effects such as dust settling. The pressure scale height is anchored at the outer disk edge, in this case $200 \mathrm{AU}$. In some cases, some degree of settling appears to have taken place. We present this number as a fraction of the scale height predicted from hydrostatic equilibrium, assuming a midplane temperature of $20 \mathrm{~K}$. In cases where the disk has settled, a flaring index of 2/7 (0.29) is likely too large (see, e.g., Andrews et al. 2009, where flaring indices range from 0.04 to 
Table 5

Optical and Near-IR Photometry for the Cold Disk Candidate Sample

\begin{tabular}{|c|c|c|c|c|c|c|c|c|}
\hline No. & $\begin{array}{c}U \\
(\mathrm{mag})\end{array}$ & $\begin{array}{c}B \\
(\mathrm{mag})\end{array}$ & $\begin{array}{c}V \\
(\mathrm{mag})\end{array}$ & $\begin{array}{c}R_{C} \\
(\mathrm{mag})\end{array}$ & $\begin{array}{c}I_{C} \\
(\mathrm{mag})\end{array}$ & $\begin{array}{c}J_{2 \text { MASS }} \\
(\mathrm{mag})\end{array}$ & $\begin{array}{c}H_{2 \mathrm{MASS}} \\
(\mathrm{mag})\end{array}$ & $\begin{array}{c}K_{\text {2MASS }} \\
(\mathrm{mag})\end{array}$ \\
\hline 1 & $\ldots$ & $\ldots$ & $\ldots$ & $\ldots$ & $\ldots$ & $15.66 \pm 0.05$ & $14.41 \pm 0.05$ & $13.69 \pm 0.05$ \\
\hline 2 & $\ldots$ & $\ldots$ & $\ldots$ & $\ldots$ & $\ldots$ & $13.76 \pm 0.05$ & $11.92 \pm 0.05$ & $11.08 \pm 0.05$ \\
\hline 3 & $\ldots$ & $\ldots$ & $\ldots$ & $\ldots$ & $\ldots$ & $15.64 \pm 0.05$ & $14.64 \pm 0.05$ & $14.37 \pm 0.05$ \\
\hline 4 & $\ldots$ & $\ldots$ & $\ldots$ & $\ldots$ & $\ldots$ & $15.52 \pm 0.05$ & $14.25 \pm 0.05$ & $13.57 \pm 0.05$ \\
\hline 5 & $\ldots$ & $\ldots$ & $\ldots$ & $\ldots$ & $\ldots$ & $12.59 \pm 0.05$ & $11.37 \pm 0.05$ & $10.96 \pm 0.05$ \\
\hline 6 & $\ldots$ & $\ldots$ & $\ldots$ & $\ldots$ & $\ldots$ & $14.86 \pm 0.05$ & $13.16 \pm 0.05$ & $11.90 \pm 0.05$ \\
\hline 7 & $\ldots$ & $\ldots$ & $\ldots$ & $\ldots$ & $\ldots$ & $15.66 \pm 0.09$ & $14.40 \pm 0.06$ & $13.69 \pm 0.05$ \\
\hline 8 & $\ldots$ & $\ldots$ & $\ldots$ & $\ldots$ & $\ldots$ & $15.57 \pm 0.05$ & $14.65 \pm 0.05$ & $14.04 \pm 0.05$ \\
\hline 9 & $\ldots$ & $\ldots$ & $\ldots$ & $\ldots$ & $\ldots$ & $11.29 \pm 0.05$ & $10.05 \pm 0.05$ & $9.41 \pm 0.05$ \\
\hline 10 & $\ldots$ & $\ldots$ & $\ldots$ & $\ldots$ & $16.33 \pm 0.01$ & $13.38 \pm 0.05$ & $11.86 \pm 0.05$ & $11.15 \pm 0.05$ \\
\hline 11 & $\ldots$ & $\ldots$ & $\ldots$ & $20.45 \pm 0.05$ & $17.93 \pm 0.05$ & $14.38 \pm 0.05$ & $12.87 \pm 0.05$ & $12.11 \pm 0.05$ \\
\hline 12 & $\ldots$ & $\ldots$ & $\ldots$ & $\ldots$ & $\ldots$ & $13.02 \pm 0.05$ & $12.09 \pm 0.05$ & $11.63 \pm 0.05$ \\
\hline 13 & $\ldots$ & $\ldots$ & $\ldots$ & $\ldots$ & $\ldots$ & $15.64 \pm 0.05$ & $13.37 \pm 0.05$ & $12.00 \pm 0.05$ \\
\hline 14 & $\ldots$ & $\ldots$ & $\ldots$ & $\ldots$ & $15.78 \pm 0.05$ & $13.07 \pm 0.05$ & $11.85 \pm 0.05$ & $11.28 \pm 0.05$ \\
\hline 15 & $\ldots$ & $\ldots$ & $\ldots$ & $\ldots$ & $\ldots$ & $10.46 \pm 0.05$ & $9.46 \pm 0.05$ & $8.93 \pm 0.05$ \\
\hline 16 & $\ldots$ & $\ldots$ & $\ldots$ & $\ldots$ & $\ldots$ & $10.15 \pm 0.05$ & $9.33 \pm 0.05$ & $8.88 \pm 0.05$ \\
\hline 17 & $\ldots$ & $17.54 \pm 0.03$ & $16.06 \pm 0.11$ & $14.53 \pm 0.04$ & $12.94 \pm 0.02$ & $10.93 \pm 0.02$ & $10.20 \pm 0.02$ & $9.85 \pm 0.02$ \\
\hline 18 & $\ldots$ & $17.51 \pm 0.03$ & $16.31 \pm 0.01$ & $15.79 \pm 0.05$ & $13.90 \pm 0.05$ & $11.94 \pm 0.05$ & $11.27 \pm 0.05$ & $10.91 \pm 0.05$ \\
\hline 19 & $\ldots$ & $\ldots$ & $12.04 \pm 0.01$ & $11.28 \pm 0.05$ & $10.54 \pm 0.05$ & $9.55 \pm 0.05$ & $8.78 \pm 0.05$ & $8.56 \pm 0.05$ \\
\hline 20 & $\ldots$ & $\ldots$ & $\ldots$ & $19.59 \pm 0.04$ & $\ldots$ & $13.59 \pm 0.05$ & $12.35 \pm 0.05$ & $11.69 \pm 0.05$ \\
\hline 21 & $\ldots$ & $19.18 \pm 0.03$ & $17.74 \pm 0.01$ & $17.03 \pm 0.04$ & $\ldots$ & $14.66 \pm 0.05$ & $13.87 \pm 0.05$ & $13.57 \pm 0.05$ \\
\hline 22 & $\ldots$ & $\ldots$ & $15.74 \pm 0.50$ & $14.82 \pm 0.05$ & $12.51 \pm 0.02$ & $10.37 \pm 0.05$ & $9.43 \pm 0.05$ & $9.06 \pm 0.05$ \\
\hline 23 & $\ldots$ & $\ldots$ & $\ldots$ & $16.22 \pm 0.04$ & $\ldots$ & $11.49 \pm 0.05$ & $10.68 \pm 0.05$ & $10.29 \pm 0.05$ \\
\hline 24 & $\ldots$ & $18.38 \pm 0.05$ & $16.59 \pm 0.05$ & $15.55 \pm 0.05$ & $\ldots$ & $11.06 \pm 0.05$ & $10.03 \pm 0.05$ & $9.51 \pm 0.05$ \\
\hline 25 & $\ldots$ & $15.80 \pm 0.75$ & $14.00 \pm 0.50$ & $12.00 \pm 0.05$ & $10.05 \pm 0.90$ & $8.09 \pm 0.02$ & $6.86 \pm 0.05$ & $6.23 \pm 0.02$ \\
\hline 26 & $\ldots$ & $\ldots$ & $\ldots$ & $\ldots$ & $\ldots$ & $14.99 \pm 0.05$ & $12.76 \pm 0.05$ & $11.81 \pm 0.05$ \\
\hline 27 & $\ldots$ & $\ldots$ & $\ldots$ & $\ldots$ & $\ldots$ & $10.72 \pm 0.05$ & $9.83 \pm 0.05$ & $9.45 \pm 0.05$ \\
\hline 28 & $\ldots$ & $\ldots$ & $\ldots$ & $\ldots$ & $\ldots$ & $15.20 \pm 0.05$ & $12.90 \pm 0.05$ & $11.55 \pm 0.05$ \\
\hline 29 & $\ldots$ & $\ldots$ & $\ldots$ & $\ldots$ & $\ldots$ & $14.03 \pm 0.05$ & $12.89 \pm 0.05$ & $12.42 \pm 0.05$ \\
\hline 30 & $\ldots$ & $\ldots$ & $\ldots$ & $\ldots$ & $\ldots$ & $14.53 \pm 0.05$ & $12.65 \pm 0.05$ & $11.69 \pm 0.05$ \\
\hline 31 & $\ldots$ & $\ldots$ & $\ldots$ & $18.38 \pm 0.05$ & $\cdots$ & $14.14 \pm 0.05$ & $12.79 \pm 0.05$ & $12.13 \pm 0.05$ \\
\hline 32 & $\ldots$ & $\ldots$ & $\ldots$ & $\ldots$ & $\ldots$ & $12.00 \pm 0.05$ & $10.84 \pm 0.05$ & $10.26 \pm 0.05$ \\
\hline 33 & $\ldots$ & $\ldots$ & $\ldots$ & $\ldots$ & $\ldots$ & $13.27 \pm 0.05$ & $11.92 \pm 0.05$ & $11.31 \pm 0.05$ \\
\hline 34 & $\ldots$ & $\ldots$ & $\ldots$ & $19.04 \pm 0.50$ & $\ldots$ & $12.19 \pm 0.05$ & $11.15 \pm 0.05$ & $10.77 \pm 0.05$ \\
\hline 35 & $\ldots$ & $\ldots$ & $\ldots$ & $19.14 \pm 0.05$ & $\ldots$ & $13.45 \pm 0.05$ & $12.11 \pm 0.05$ & $11.45 \pm 0.05$ \\
\hline
\end{tabular}

References. Optical fluxes come from (1) H. Bouy et al. 2010, in preparation for Perseus sources (1-15); (2) Spezzi et al. 2008 for the only object from Chamaeleon II (16); (3) Comerón et al. 2009 for the Lupus sources (17 and 18); (4) H. Bouy et al. 2010, in preparation for Ophiuchus sources (19-25); and (5) Spezzi et al. 2010 for the sources in Serpens (26-35).

0.26). Disk model SEDs with large flaring indices are similar to cold disk SEDs in having more flux at longer wavelengths. By using a large flaring index as our default, we are more conservative when reporting the presence of a hole. Surface density is a power law of the radius, with an index of -1 inside $200 \mathrm{AU}$, which steepens to an index of -12 outside the $200 \mathrm{AU}$ outer disk edge. The dust composition is set to a silicate:carbon ratio of 3:1 with only amorphous, as opposed to crystalline, silicate included. Silicate opacities were taken from Beckwith et al. (1990), who provide opacities in the millimeter. Disk masses were taken from $1.3 \mathrm{~mm}$ observations where possible (see Table 7) and left as a free parameter otherwise. Disk mass and disk settling are highly degenerate parameters in the absence of (sub)millimeter fluxes, so when the disk mass is unknown the scale height is left at the hydrostatic value (see notes on exceptions for sources 18, 19, and 24 in Section 4.4). As a result, model-derived disk masses tend to be systematically small and together with observational upper limits provide a range of likely disk masses. The inner edge of the disk, when no hole is present, was set at approximately the radius where the dust sublimation temperature, $1500 \mathrm{~K}$, is reached. Some cold disks in the literature have near-IR excesses most easily modeled as a gap rather than a hole (e.g., Brown et al. 2007; Espaillat et al. 2008). However, none of the sources in this sample required warm dust within the hole. Those sources that did have some near-IR excess turned out to be compatible with disk models without holes. The hole is represented in the model by a hole radius, $R_{\text {Hole }}$, and a density reduction, set here to a factor of $10^{-6}$, providing an almost entirely clean hole. Thus, the four free parameters are $R_{\text {Hole }}$, degree of settling, disk inclination, and disk mass when there are no measurements.

A two-step procedure was used to find the best model fits. First, we ran a large grid of disk models with a broad range of disk and hole parameters and a limited number of stellar templates. Then, we ran a finer grid of disk and hole parameters, determined from the larger grid, for each individual star using the stellar properties in Table 4. A simple $\chi^{2}$ minimization was performed at each step between the dereddened SEDs and the model SEDs and the resulting best fit is shown as a red dashdotted line in the SED plots (Figures 7 and 9). Errors on the hole radii are given in Table 7 based on fits with up to $20 \%$ deviation in $\chi^{2}$. 
Table 6

Selection Criteria for Edge-on Disks

\begin{tabular}{rccccc}
\hline \hline No. & Underluminous & $\begin{array}{c}\text { Forbidden } \\
\text { Lines }\end{array}$ & $\begin{array}{c}\text { Silicate } \\
\text { Absorption }\end{array}$ & $\begin{array}{c}\text { Ice } \\
\text { Absorption }\end{array}$ & $\begin{array}{c}\text { High } \\
L_{\text {Disk }} / L_{*}\end{array}$ \\
\hline 1 & $\checkmark$ & & & & $\checkmark$ \\
3 & $\checkmark$ & $\checkmark ?$ & $\checkmark$ & $\checkmark$ & \\
4 & & $\checkmark$ & $\checkmark$ & \\
6 & & $\checkmark$ & & & $\checkmark$ \\
7 & $\checkmark$ & $\checkmark$ & & $\checkmark$ \\
8 & $\checkmark$ & $\checkmark$ & & & $\checkmark$ \\
21 & $\checkmark$ & & & & $\checkmark$ \\
31 & & & & & \\
\hline
\end{tabular}

Fifteen of the twenty-five modeled stars require inner holes, while the remaining ten require no hole to fit the SED. The ten sources not modeled are the eight edge-on disks and two sources, 25 and 27, which have strong extended $70 \mu \mathrm{m}$ emission and cannot be fit with disk models (see Section 4.4 for further details). Hole radii range from $1 \mathrm{AU}$ to $55 \mathrm{AU}$. Holes smaller than $1 \mathrm{AU}$ cannot be firmly identified. The range of hole radii can be seen in histogram form in Figure 10.

In order to confirm our results above and identify the less model-dependent part of our results, we also fitted our sample SEDs with the online SED fitting tool by Robitaille et al. (2007). This system offers the possibility of fitting young star SEDs with a precomputed grid of 200,000 synthetic SEDs from stars, disks, and envelopes with a broad range of physical parameters. In order to make these fits, we observed photometry from Tables 2 and 5 along with a binned IRS spectrum from 6 to $33 \mu \mathrm{m}$ with steps of $1 \mu \mathrm{m}$. An aperture of $4 . .5$, a distance range from 0.1 to $0.33 \mathrm{kpc}$, and an $A_{V}$ range from 0 to 30 mag were used in all fits. For the objects for which the spectral types are known, we select the best-fitting model with an effective temperature less than $500 \mathrm{~K}$ away from the target's temperature. The final best-fit models used are good representations of the median properties of the best-fitting models with the appropriate effective temperatures but should not be considered as definitively constrained parameters.

Figures 7 and 8 show the Robitaille et al. best-fit models for the objects in our sample as thin black dashed lines and Table 7 shows their corresponding model IDs and the inner disk radii in the cases where it is clearly distinguishable from the dust destruction inner disk radius. In order to overplot the Robitaille et al. models, we corrected them with the extinction law given in the Web-fitting tool and using the total $A_{V}$ value given by the best-fit model and normalized it to the $K_{2 \mathrm{MASS}}{ }^{-}$ band dereddened flux. The $A_{V}$ values of the best-fit models used are, in general, in agreement with the $A_{V}$ values obtained from the spectral type and optical photometry. However, small discrepancies plus small differences in the standard extinction laws used to deredden our photometry and the Robitaille models can explain the discrepancies between the Robitaille models and optical fluxes in some cases.

All but one of the cold disks in Figure 7 also have inner holes with the Robitaille et al. models. The only exception, source 18 , is a borderline case with an extremely small inner cavity of $0.03 \mathrm{AU}$ in the Robitaille et al. best-fit model. On the other hand, almost all the objects in Figure 9, which have no hole according to the RADMC models, show inner cavities smaller than $2 \mathrm{AU}$ in the Robitaille models. The only exception is source 15, which is also among the flattest disks in the group. This indicates that, in general terms, the classification of objects as disks with or without inner holes is model independent as long as the disk hole is larger than $2 \mathrm{AU}$.

Figure 11 compares the disk inner holes estimated both with the RADMC and the Robitaille et al. (2007) disk model grids. The plot does not include the only outlier (source 18). For the rest of the sample, the values from both sets of models are correlated, but with a very large scatter. It must be noted that this comparison is not straightforward as both sets of models have different treatments of the dust opacities, disk flaring angle, and inner hole structure. Also, the Robitaille et al. online tool does not allow the use of a priori information like the lack of an envelope, or the effective temperature of the central star, both of which obviously have great impact on the final result of the fitting. Pursuing a more detailed comparison between both sets of disk models is beyond the scope of this paper, but this comparison shows that the derived disk hole radius is model independent to within a factor of 2-3 generally. We find the RADMC models more reliable due to the greater control over the input parameters and the resulting better fits. The RADMC disk radii are therefore used in all further comparisons.

\subsection{Dust Composition}

A wide variety of mid-IR spectral features have been detected in spectra of disks around low- and intermediate-mass young stars using the Infrared Space Observatory (e.g., Acke \& van den Ancker 2004) and Spitzer (e.g., Kessler-Silacci et al. 2006; Furlan et al. 2006; Lahuis et al. 2007; Bouwman et al. 2008; Watson et al. 2009; Olofsson et al. 2009). These features can serve as diagnostics of physical processes in disks such as grain growth, fragmentation, crystallization, flaring, and UV or $\mathrm{X}$-ray illumination. An interesting question is whether the spectral features of cold disks differ significantly from those of normal T Tauri disks, and, if so, what that tells us about the disk structure and evolution.

Table 8 identifies the presence or absence of the 10 and $20 \mu \mathrm{m}$ silicate emission features, polycyclic aromatic hydrocarbons (PAHs), and crystalline silicate features, where "Y" means a detection, "N" a non-detection, and " $\mathrm{T}$ " a tentative detection. The spectra presented in Figures 1-5 clearly show a large variety of features in our sample of cold disks.

\subsubsection{Silicates}

The silicate 10 and $20 \mu \mathrm{m}$ features are detected in most cases; none of the sources show the featureless spectra commonly seen for older (debris) disks (e.g., Jura et al. 2004; Chen et al. 2006; Carpenter et al. 2009). However, only a few sources have pristine ISM-like $10 \mu \mathrm{m}$ silicate features, characterized by a strongly peaked profile centered at $9.8 \mu \mathrm{m}$. This is illustrated in Figure 12 in which the $S_{11.3} / S_{9.8} \mu \mathrm{m}$ flux ratio is plotted versus the strength of the feature above the continuum $S_{\text {peak }}^{10 \mu \mathrm{m}}$, as defined in KesslerSilacci et al. (2006). The different quantities $S_{11.3}, S_{9.8}$, and $S_{\text {Peak }}^{10 \mu \mathrm{m}}$ are calculated after normalizing the observed feature as follows: $S_{v}=1+\left(F_{v}-F_{v, \text { cont }}\right) /\left(\left\langle F_{v, \text { cont }}\right\rangle\right)$, where $F_{v, \text { cont }}$ is the local continuum and $\left\langle F_{v, \text { cont }}\right\rangle$ is the mean value of this continuum. The local continuum is estimated using a seconddegree polynomial, normalized at $6.8-7.5 \mu \mathrm{m}, 12.5-13.5 \mu \mathrm{m}$, and $30-36 \mu \mathrm{m}$. The fluxes are measured as an averaged flux of the continuum-subtracted spectra in a $\pm 0.1 \mu \mathrm{m}$ interval at each wavelength.

As shown by van Boekel et al. (2005) and Olofsson et al. (2009), sources in which the grain size distribution is dominated by large $\mu \mathrm{m}$ sized grains, or sources with size distributions much 
Table 7

Disk Physical Parameters and Observables

\begin{tabular}{|c|c|c|c|c|c|c|c|c|c|c|c|c|}
\hline No. & Reg. & $\begin{array}{l}R_{\text {hole }^{\mathrm{a}}} \\
(\mathrm{AU})\end{array}$ & $\begin{array}{l}\text { Inc. } \\
\text { (deg) }\end{array}$ & $\begin{array}{c}\text { Mass }^{\mathrm{b}} \\
\left(10^{-3} M_{\odot}\right)\end{array}$ & Sett. & $\begin{array}{c}F_{\mathrm{mm}} \\
(\mathrm{mJy})\end{array}$ & $\begin{array}{c}M_{\text {disk }} \\
\left(10^{-3} M_{\odot}\right)\end{array}$ & Ref. & $F_{30} / F_{13}$ & $L_{\text {disk }} / L_{*}$ & $\begin{array}{l}R_{\text {hole }}^{\text {Rob. }} \\
\text { (AU) }\end{array}$ & $\begin{array}{l}\text { Model ID } \\
\text { (Rob.) }\end{array}$ \\
\hline 1 & $\ldots$ & Edge & $\ldots$ & $\ldots$ & $\ldots$ & $<1.0$ & $<0.3$ & 1 & 20.17 & $\ldots$ & $\ldots$ & $\ldots$ \\
\hline 2 & B & $1.5 \pm 1$ & 75 & Obs & 0.5 & $3.7 \pm 0.9$ & $1.1 \pm 0.3$ & 1 & 4.29 & 0.16 & 1.64 & 3005228 \\
\hline 3 & B & Edge & $\ldots$ & $\ldots$ & $\ldots$ & $\ldots$ & $\ldots$ & $\ldots$ & 21.35 & $\ldots$ & $\ldots$ & $\ldots$ \\
\hline 4 & $\ldots$ & Edge & $\ldots$ & $\ldots$ & $\ldots$ & $<1.3$ & $<0.4$ & 1 & 9.82 & $\ldots$ & $\ldots$ & $\ldots$ \\
\hline 5 & A & $6_{-2}^{+4}$ & 10 & Obs & 0.5 & $6.3 \pm 1.1$ & $1.9 \pm 0.3$ & 1 & 12.86 & 0.2 & 15.3 & 3000829 \\
\hline 6 & $\ldots$ & Edge & $\ldots$ & $\ldots$ & $\ldots$ & $<0.7$ & $<0.2$ & 1 & 17.63 & $\ldots$ & $\ldots$ & $\ldots$ \\
\hline 7 & $\mathrm{~B}$ & Edge & $\ldots$ & $\ldots$ & $\ldots$ & $1.3 \pm 0.5$ & $0.4 \pm 0.2$ & 1 & 17.47 & $\ldots$ & $\ldots$ & $\ldots$ \\
\hline 8 & $\ldots$ & Edge & $\ldots$ & $\ldots$ & $\ldots$ & $\ldots$ & $\ldots$ & $\ldots$ & 33.19 & $\ldots$ & $\ldots$ & $\ldots$ \\
\hline 9 & B & No hole & 30 & Obs & 0.5 & $13.0 \pm 1.3$ & $4.0 \pm 0.4$ & 1 & 3.01 & 0.19 & $\ldots$ & $\ldots$ \\
\hline 10 & A & $5 \pm 1$ & 60 & 0.001 & 1 & $<1.4$ & $<0.4$ & 1 & 4.76 & 0.04 & 1.87 & 3018041 \\
\hline 11 & A & $5 \pm 1$ & 30 & 0.003 & 1 & $\ldots$ & $\ldots$ & $\ldots$ & 7.85 & 0.22 & 0.75 & 3015642 \\
\hline 13 & $\ldots$ & No hole & 45 & Obs & 1 & $11.6 \pm 1.0$ & $3.5 \pm 0.3$ & 1 & 4.51 & 0.18 & 1.71 & 3007226 \\
\hline 12 & A & $3 \pm 1$ & 5 & 0.002 & 1 & $<1.4$ & $<0.4$ & 1 & 5.9 & 0.2 & 1.04 & 3017747 \\
\hline 14 & B & No hole & 60 & 0.3 & 1 & $<1.5$ & $<0.5$ & 1 & 5.78 & 0.23 & 0.8 & 3019220 \\
\hline 15 & B & No hole & 75 & 0.005 & 1 & $<1.2$ & $<0.4$ & 1 & 2.38 & 0.05 & 8.6 & 3005263 \\
\hline 16 & $\mathrm{~B}$ & No hole & 65 & 0.05 & 1 & $\ldots$ & $\ldots$ & $\ldots$ & 2.65 & 0.12 & $\ldots$ & 3012969 \\
\hline 17 & $\ldots$ & $55 \pm 5$ & 45 & 0.03 & 1 & $<35.9$ & $<4.0$ & 2 & 11.47 & 0.15 & 96.0 & 3004704 \\
\hline 18 & A & $2 \pm 1$ & 75 & 0.5 & 0.05 & $\ldots$ & $\ldots$ & $\ldots$ & 4.67 & 0.09 & $\ldots$ & 3000691 \\
\hline 19 & A & $2 \pm 1$ & 5 & 0.5 & 0.1 & $\ldots$ & $\ldots$ & $\ldots$ & 5.36 & 0.09 & 3.8 & 3017818 \\
\hline 20 & $\ldots$ & No hole & 5 & 0.01 & 1 & $<4.6$ & $<0.3$ & 1 & 3.82 & 0.33 & 0.8 & 3019048 \\
\hline 21 & $\ldots$ & Edge & $\ldots$ & $\ldots$ & $\ldots$ & $<4.4$ & $<0.3$ & 1 & 5.55 & $\ldots$ & $\ldots$ & $\ldots$ \\
\hline 22 & A & $1_{-0.5}^{+1}$ & 30 & 0.25 & 1 & $<5.4$ & $<0.4$ & 1 & 3.16 & 0.18 & 2.75 & 3010142 \\
\hline 23 & B & No hole & 80 & 0.004 & 1 & $\ldots$ & $\ldots$ & $\ldots$ & 3.55 & 0.19 & 0.3 & 3004623 \\
\hline 24 & A & $3 \pm 2$ & 5 & 0.1 & 0.25 & $\ldots$ & $\ldots$ & $\ldots$ & 6.98 & 0.13 & 2.21 & 3005973 \\
\hline 25 & A & Back & $\ldots$ & $\ldots$ & $\ldots$ & $\ldots$ & $\ldots$ & $\ldots$ & 5.67 & 0.12 & $\ldots$ & $\ldots$ \\
\hline 26 & A & $4 \pm 1$ & 5 & Obs & 0.15 & $24.0 \pm 0.6$ & $8.0 \pm 0.2$ & 1 & 8.87 & 0.17 & 17.6 & 3002156 \\
\hline 27 & $\ldots$ & Back & $\ldots$ & $\ldots$ & $\ldots$ & $\ldots$ & $\ldots$ & $\ldots$ & 1.28 & 0.03 & $\ldots$ & $\ldots$ \\
\hline 28 & $\ldots$ & No hole & 5 & 0.002 & 1 & $\ldots$ & $\ldots$ & $\ldots$ & 2.41 & 0.15 & $\ldots$ & 3008873 \\
\hline 29 & A & $8 \pm 2$ & 30 & 0.01 & 1 & $\ldots$ & $\ldots$ & $\ldots$ & 11.71 & 0.07 & 3.76 & 3010258 \\
\hline 30 & $\ldots$ & No hole & 45 & 0.05 & 1 & $\ldots$ & $\ldots$ & $\ldots$ & 2.53 & 0.03 & 0.5 & 3006320 \\
\hline 31 & $\ldots$ & Edge & 81.4 & $\ldots$ & $\ldots$ & $\ldots$ & $\ldots$ & $\ldots$ & 5.89 & $\ldots$ & $\ldots$ & $\ldots$ \\
\hline 32 & A & $7 \pm 1$ & 5 & 0.01 & 1 & $\ldots$ & $\ldots$ & $\ldots$ & 6.01 & 0.11 & 4.14 & 3008745 \\
\hline 33 & A & $13 \pm 3$ & 5 & Obs & 0.005 & $2.3 \pm 0.6$ & $0.8 \pm 0.2$ & 1 & 7.36 & 0.33 & 3.94 & 3018536 \\
\hline 34 & A & $25_{-5}^{+15}$ & 5 & Obs & 0.25 & $6.3 \pm 0.6$ & $2.1 \pm 0.2$ & 1 & 10.63 & 0.02 & 8.94 & 3019329 \\
\hline 35 & $\cdots$ & No hole & 80 & Obs & 0.025 & $2.2 \pm 0.9$ & $0.7 \pm 0.3$ & 1 & 1.5 & 0.13 & 0.4 & 3016446 \\
\hline
\end{tabular}

Notes. Column "Reg." gives the regions in the upper left panel of Figure 15.

a "Edge" labels edge-on disks; "Back" labels the two SEDs contaminated by background cloud material.

b "Obs" indicates that the disk mass in the model is the observed millimeter mass.

References. (1) Continuum flux at $1.3 \mathrm{~mm}$ from this work (Section 2.4); (2) Nuernberger et al. 1997.

flatter than the MRN (the classical grain size distribution of the interstellar medium, $d n(a) \propto a^{p} d a$, with $a$ being the grain size and $p=-3.5$; Mathis et al. 1977) size distribution, appear in the upper left corner of the plot, whereas those dominated by smaller ISM-type grains are located in the lower right part. For comparison, the silicate profile parameters of the c2d sample studied by Kessler-Silacci et al. (2006) and Olofsson et al. (2009) are overplotted (gray dots). It is seen that the cold disks fall mostly in the upper left part of the figure, indicating that the dust in these disks has already undergone non-negligible grain growth. No significant difference was found in the $10 \mu \mathrm{m}$ shape for accreting or non-accreting objects. This conclusion is not affected by crystallization or sedimentation of dust toward the midplane, which causes some spread in the relation but cannot reproduce the observed trend, as discussed extensively in Dullemond \& Dominik (2008) and Olofsson et al. (2009).

Some confirmed disks with inner holes show crystalline silicate features, either the forsterite feature at $33 \mu \mathrm{m}$ and/or the 23 and $28 \mu \mathrm{m}$ complexes (see Olofsson et al. 2009 for definitions of these features). The fraction is between $33 \%$ and $60 \%$ $(5 / 15$ to $9 / 15)$ with the range due to some tentative detections limited by the low $\mathrm{S} / \mathrm{N}$ in some spectra. A prominent example of a highly crystalline spectrum is that of object 10, SSTc2d J034227.1+314433, a cold disk in Perseus (see Figure 5). The detection frequency of this crystalline material is comparable to the $55 \%$ found for the c2d sample of normal CTTS (Classical T Tauri Star) disks based on long-wavelength features, whereas Watson et al. (2009) claim an even higher fraction of $94 \%$ for normal disks in Taurus (see discussion in Olofsson et al. 2009).

\subsubsection{PAHs}

The detection of PAHs is based on at least two of the features at 6.2, 7.7, 8.6, 11.2, 12.8, and $16.4 \mu \mathrm{m}$ (Koike et al. 1993; Geers et al. 2006; Tielens 2008). Overall, PAH features are detected in $13 \%(2 / 15)$ of the cold disks in our sample. This is similar to the fraction of $11 \%-14 \%$ found by Geers et al. (2006) for a sample of normal CTTS disks (also see Geers et al. 2007b). This low detection fraction not only reflects the fact that late-type stars have less UV and optical photons to excite the PAHs but also implies a PAH abundance in disks that is typically a factor of $10-100$ lower than in the normal ISM, taken to be $5 \times 10^{-7}$ with 
Table 8

Classification and Mineralogy of the Disk Sample

\begin{tabular}{|c|c|c|c|c|c|c|c|c|}
\hline No. & Name & Hole & Acc. & $M_{\text {disk }}$ & $10 \mu \mathrm{m}$ & $20 \mu \mathrm{m}$ & $\mathrm{PAH}$ & Cryst. \\
\hline 2 & ASR118 & $\mathrm{Y}$ & $\mathrm{Y}$ & $\mathrm{L}$ & $\mathrm{Y}$ & $\mathrm{Y}$ & $\mathrm{N}$ & $\mathrm{T}$ \\
\hline 5 & SSTc2d J032929.3+3118 & $\mathrm{Y}$ & $\mathrm{Y}$ & $\mathrm{L}$ & $\mathrm{Y}$ & $\mathrm{Y}$ & $\mathrm{N}$ & $\mathrm{T}$ \\
\hline 10 & SSTc2d J034227.1+3144 & $\mathrm{Y}$ & $\mathrm{N}$ & $\mathrm{L}$ & $\mathrm{Y}$ & $\mathrm{N}$ & $\mathrm{N}$ & $\mathrm{Y}$ \\
\hline 11 & Cl* IC 348 LRL 190 & $\mathrm{Y}$ & $\mathrm{N}$ & $\ldots$ & Y & $\mathrm{Y}$ & $\mathrm{Y}$ & $\mathrm{N}$ \\
\hline 12 & Cl* IC 348 LRL 265 & $\mathrm{Y}$ & $\mathrm{Y}$ & $\mathrm{L}$ & Y & $\mathrm{Y}$ & $\mathrm{N}$ & Y \\
\hline 17 & $\mathrm{Sz} 84$ & $\mathrm{Y}^{\mathrm{a}}$ & $\mathrm{Y}$ & $\mathrm{L}$ & $\mathrm{N}$ & $\mathrm{N}$ & $\mathrm{N}$ & $\mathrm{N}$ \\
\hline 18 & SSTc2d J161029.6-3922 & $\mathrm{Y}$ & $\ldots$ & $\ldots$ & $\mathrm{Y}$ & $\mathrm{Y}$ & $\mathrm{N}$ & $\mathrm{Y}$ \\
\hline 19 & RX J1615.3-3255 & $\mathrm{Y}$ & $\mathrm{Y}$ & $\ldots$ & Y & $\mathrm{Y}$ & $\mathrm{Y}$ & $\mathrm{N}$ \\
\hline 22 & SSTc2d J162245.4-2431 & $\mathrm{Y}$ & $\mathrm{N}$ & $\mathrm{L}$ & $\mathrm{Y}$ & $\mathrm{Y}$ & $\mathrm{N}$ & $\mathrm{N}$ \\
\hline 24 & SSTc2d J162506.9-2350 & $\mathrm{Y}$ & $\mathrm{Y}$ & $\ldots$ & $\mathrm{Y}$ & $\mathrm{Y}$ & $\mathrm{N}$ & $\mathrm{Y}$ \\
\hline 26 & SSTc2d J182829.1+0027 & $\mathrm{Y}$ & $\ldots$ & $\mathrm{H}$ & $\mathrm{Y}$ & $\mathrm{Y}$ & $\mathrm{N}$ & $\mathrm{T}$ \\
\hline 29 & SSTc2d J182911.5+0020 & $\mathrm{Y}$ & $\mathrm{Y}$ & $\ldots$ & Y & $\mathrm{Y}$ & $\mathrm{N}$ & $\mathrm{N}$ \\
\hline 32 & SSTc2d J182935.6+0035 & $\mathrm{Y}$ & $\mathrm{Y}$ & $\ldots$ & $\mathrm{Y}$ & Y & $\mathrm{N}$ & $\mathrm{Y}$ \\
\hline 33 & SSTc2d J182936.2+0042 & $\mathrm{Y}$ & $\ldots$ & $\mathrm{L}$ & $\mathrm{Y}$ & $\mathrm{Y}$ & $\mathrm{N}$ & $\mathrm{T}$ \\
\hline 34 & SSTc2d J182944.1+0033 & $\mathrm{Y}$ & $\mathrm{Y}$ & $\mathrm{L}$ & $\mathrm{Y}$ & $\mathrm{N}$ & $\mathrm{N}$ & $\mathrm{N}$ \\
\hline 1 & SSTc2d J032835.0+3020b & $\mathrm{N}$ & $\mathrm{Y}$ & $\mathrm{L}$ & $\mathrm{Y}$ & $\mathrm{Y}$ & $\mathrm{N}$ & $\mathrm{N}$ \\
\hline 3 & SSTc2d J032903.9+3056 & $\mathrm{N}$ & $\mathrm{Y}$ & $\ldots$ & $\mathrm{N}$ & $\mathrm{Y}$ & Y & Y \\
\hline 4 & SSTc2d J032924.1+3119 & $\mathrm{N}$ & $\mathrm{N}$ & $\mathrm{L}$ & $\mathrm{N}$ & $\mathrm{Y}$ & $\mathrm{N}$ & $\mathrm{N}$ \\
\hline 6 & SSTc2d J033027.1+3028b & $\mathrm{N}$ & $\mathrm{Y}$ & $\mathrm{L}$ & $\mathrm{Y}$ & $\mathrm{Y}$ & $\mathrm{N}$ & $\mathrm{Y}$ \\
\hline 7 & SSTc2d J033035.5+3115 & $\mathrm{N}$ & $\mathrm{Y}$ & $\mathrm{L}$ & $\mathrm{N}$ & $\mathrm{Y}$ & $\mathrm{N}$ & $\mathrm{Y}$ \\
\hline 8 & SSTc2d J033038.2+3032 & $\mathrm{N}$ & $\mathrm{Y}$ & $\ldots$ & $\mathrm{N}$ & $\mathrm{Y}$ & $\mathrm{N}$ & $\mathrm{Y}$ \\
\hline 9 & LZK 21 & $\mathrm{~N}$ & $\mathrm{Y}$ & $\mathrm{L}$ & $\mathrm{Y}$ & $\mathrm{Y}$ & $\mathrm{N}$ & Y \\
\hline 13 & SSTc2d J034434.8+3156 & $\mathrm{N}$ & $\ldots$ & $\mathrm{L}$ & $\mathrm{Y}$ & $\mathrm{Y}$ & $\mathrm{N}$ & $\mathrm{T}$ \\
\hline 14 & $\mathrm{Cl}^{*}$ IC 348 LRL 110 & $\mathrm{~N}$ & Y & $\mathrm{L}$ & Y & $\mathrm{N}$ & $\mathrm{Y}$ & $\mathrm{N}$ \\
\hline 15 & $\mathrm{LkH} \alpha 329$ & $\mathrm{~N}$ & $\mathrm{Y}$ & $\mathrm{L}$ & Y & $\mathrm{Y}$ & $\mathrm{N}$ & Y \\
\hline 16 & Hn 24 & $\mathrm{~N}$ & $\mathrm{~N}$ & $\ldots$ & $\mathrm{Y}$ & $\mathrm{Y}$ & $\mathrm{N}$ & $\mathrm{Y}$ \\
\hline 20 & SSTc2d J162148.5-2340 & $\mathrm{N}$ & $\mathrm{Y}$ & $\mathrm{L}$ & $\mathrm{Y}$ & $\mathrm{Y}$ & $\mathrm{N}$ & $\mathrm{N}$ \\
\hline 21 & SSTc2d J162221.1-2304 & $\mathrm{N}$ & $\mathrm{Y}$ & $\mathrm{L}$ & $\mathrm{Y}$ & $\mathrm{Y}$ & $\mathrm{N}$ & $\mathrm{N}$ \\
\hline 23 & SSTc2d J162332.8-2258 & $\mathrm{N}$ & $\mathrm{N}$ & $\ldots$ & $\mathrm{Y}$ & $\mathrm{N}$ & $\mathrm{N}$ & $\mathrm{Y}$ \\
\hline 25 & DoAr 21 & $\mathrm{~N}$ & $\mathrm{~N}$ & $\ldots$ & $\mathrm{N}$ & $\mathrm{N}$ & $\mathrm{Y}$ & $\mathrm{N}$ \\
\hline 27 & SSTc2d J182858.1+0017 & $\mathrm{N}$ & $\mathrm{N}$ & $\ldots$ & $\mathrm{N}$ & $\mathrm{N}$ & $\mathrm{Y}$ & $\mathrm{N}$ \\
\hline 28 & SSTc2d J182907.0+0038 & $\mathrm{N}$ & $\ldots$ & $\ldots$ & $\mathrm{Y}$ & $\mathrm{N}$ & $\mathrm{Y}$ & $\mathrm{N}$ \\
\hline 30 & SSTc2d J182915.6+0039 & $\mathrm{N}$ & $\mathrm{Y}$ & $\ldots$ & $\mathrm{Y}$ & Y & $\mathrm{N}$ & $\mathrm{Y}$ \\
\hline 31 & SSTc2d J182915.6+0039 & $\mathrm{N}$ & $\ldots$ & $\ldots$ & $\mathrm{Y}$ & $\mathrm{Y}$ & $\mathrm{N}$ & $\mathrm{N}$ \\
\hline 35 & SSTc2d J182947.3+0032 & $\mathrm{N}$ & $\mathrm{Y}$ & $\mathrm{L}$ & $\mathrm{Y}$ & $\mathrm{Y}$ & $\mathrm{N}$ & $\mathrm{N}$ \\
\hline
\end{tabular}

Notes. For clarity, cold disks and other objects are shown above and below the horizontal line, respectively. "Y" indicates that the feature is present, "N" indicates that the feature is not present, and " $\mathrm{T}$ " indicates a tentative detection. "L" and " $\mathrm{H}$ " in Column 5 represent objects with total disk masses smaller and larger than $5 \times 10^{-3} M_{\odot}$, respectively.

a The classification of Sz 84 (17) as a cold disk or an extended source is ambiguous but we keep it, assuming that it might be both.

b Close to edge-on according to SED fit and luminosity below the main sequence. In these cases, we do not classify them as cold disks, since the hole size determination from the SED fit is highly uncertain.

respect to the total hydrogen for PAH species with 100 carbon atoms (e.g., $\mathrm{C}_{100} \mathrm{H}_{24}$; Geers et al. 2006). Both sources 25 and 27, which have long-wavelength fluxes composed of extended material, show exceptionally strong PAH features. Both have low continuum at mid-IR wavelengths increasing the line-tocontinuum contrast. In the case of source 27 , even the $16.4 \mu \mathrm{m}$ band is clearly revealed (see Figure 13).

The strong features seen in cold disks do not necessarily imply higher PAH abundances than in normal CTTS disks, however. Dullemond et al. (2007) model the PAH emission in disks with varying degrees of sedimentation, as characterized by the $\alpha$ parameter of turbulence. In the case of low turbulence, $\alpha<10^{-4}$, the big grains quickly sediment to the midplane but the PAHs stay in the upper layers, boosting the featureto-continuum ratio by a factor of $2-10$. For high turbulence, $\alpha \approx 10^{-2}$, the small and big grains stay well mixed in the upper layers, resulting in the typically weak or absent PAH features consistent with observations (Geers et al. 2006). Dullemond et al. (2007) did not consider disks with inner holes, but qualitatively the effect is similar to their low-turbulence case in which the big grains are removed and the feature strengths are boosted.

In contrast with the silicates, the PAH emission arises not only from the inner disk but also from the outer disk surface exposed to optical and UV radiation. Indeed, spatially extended PAH emission has been detected out to $\sim 60 \mathrm{AU}$ or more for some disks (Habart et al. 2006; Geers et al. 2007a). Two cold disks within the c2d clouds, SR 21 and T Cha, are included in the observations of Geers et al. (2007b) using VLT-ISAAC and VLT-VISIR to get higher spatial resolution as compared to Spitzer. In both cases, the emission is found to be spatially unresolved with limiting spatial extents (FWHM) of 19 and 13 AU, respectively. These limits are comparable to the values of $R_{\mathrm{Hole}}$ of 18 and $15 \mathrm{AU}$, respectively (Brown et al. 2007; Table 7), indicating that the PAHs are located primarily inside the gaps. For the case of SR 21, the location of the gas inside the gap has been pinpointed to a ring at $\sim 7 \mathrm{AU}$ radius (Pontoppidan et al. 2008), proving that the gas emission is indeed coming from 
No. 2, 2010
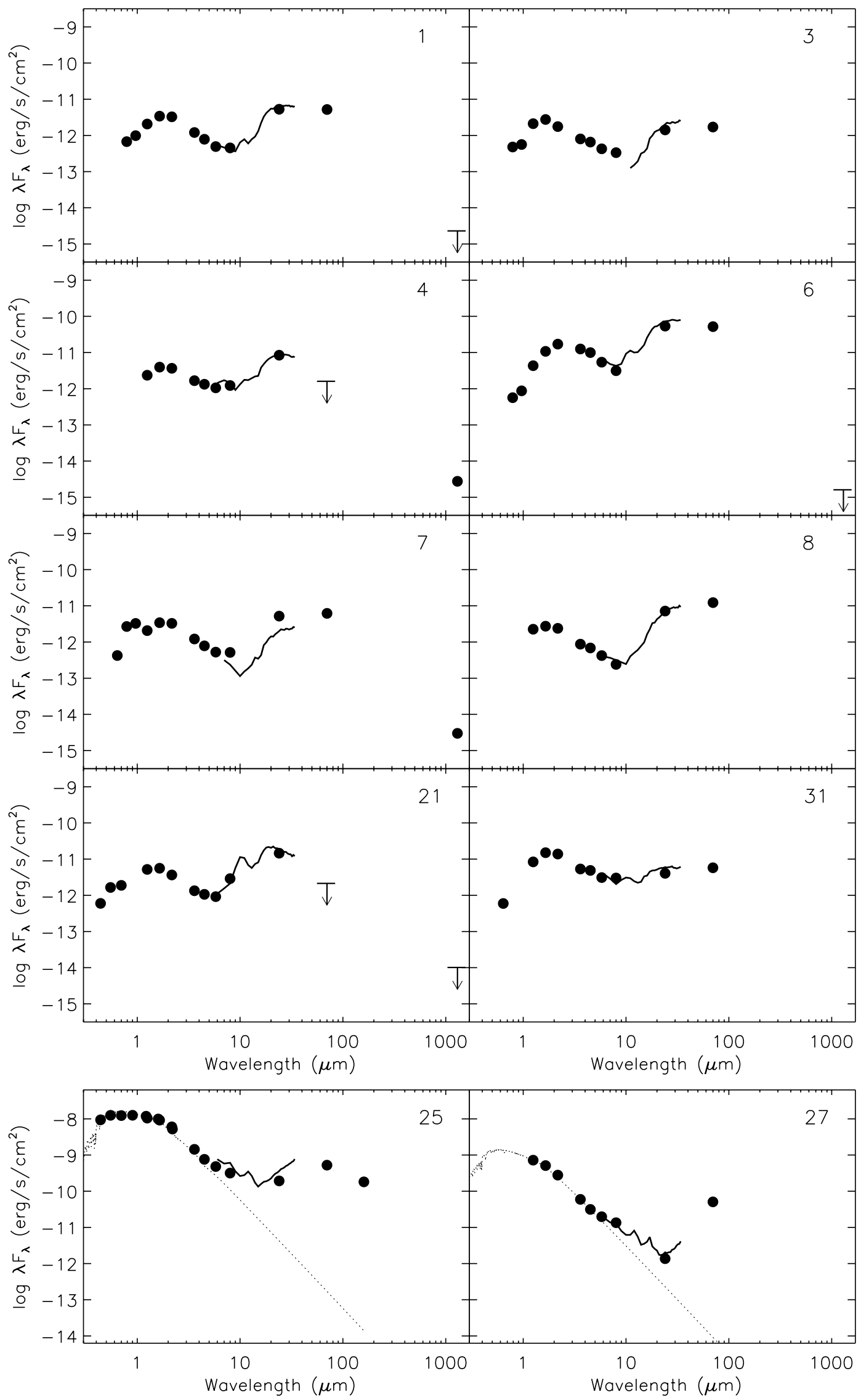

Figure 6. SEDs of the eight close to edge-on disks (top) in the sample and the two extended sources (bottom). Points are observed fluxes from IRAC, MIPS, and IRAM. The thick solid line is the IRS spectrum and the numbers give their identifications in Table 1. 


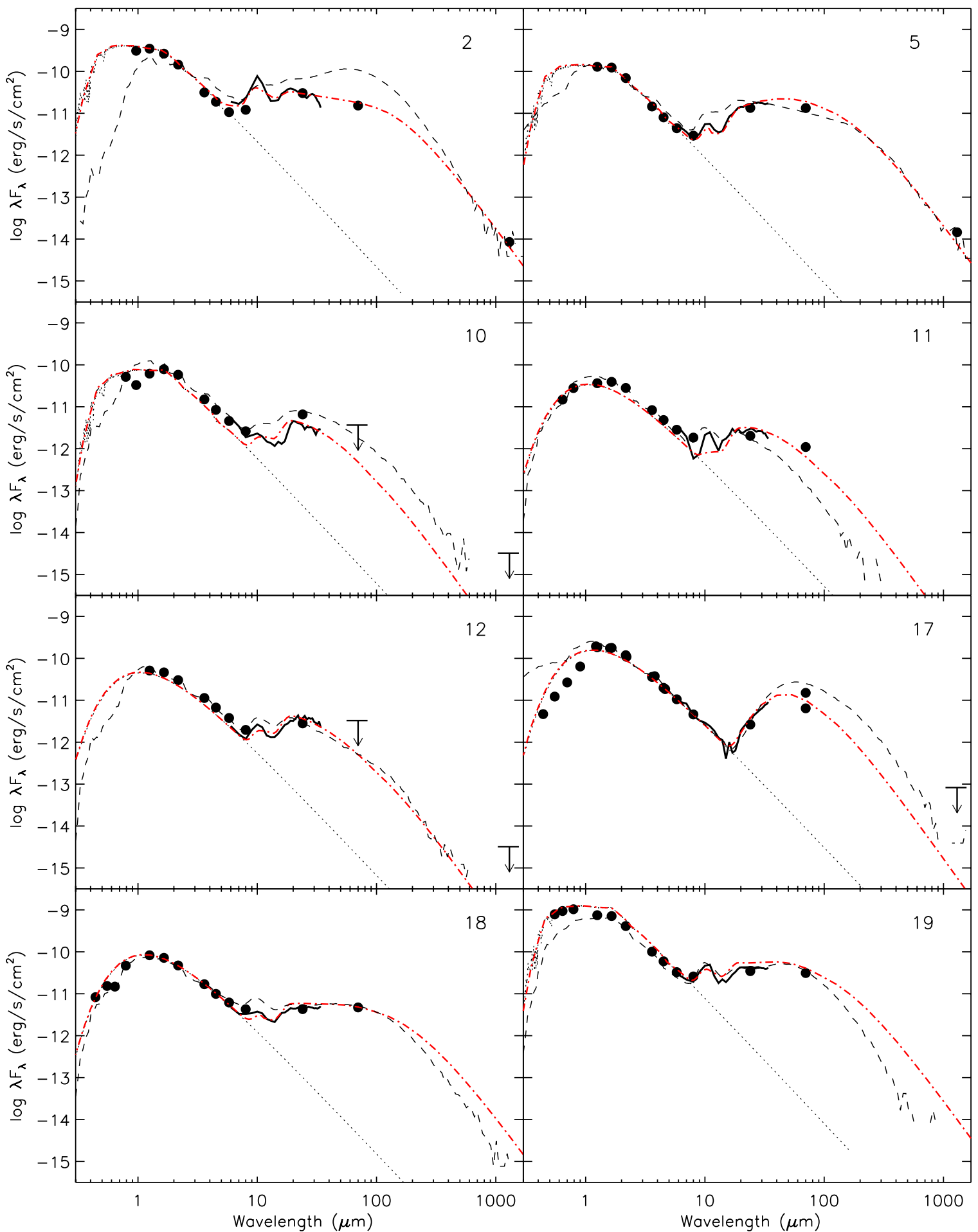

Figure 7. SEDs of the disks with holes in the sample. Points are dereddened fluxes from IRAC, MIPS, and IRAM. The thick solid line is the IRS spectrum, the dotted line is the stellar photospheric model, the red dash-dotted line is the best-fit RADMC disk model, and the thin black dashed line is the Robitaille model fit. The numbers give their identifications in Table 1.

(A color version of this figure is available in the online journal.)

inside the gap and not from the outer edge or wall of the gap. Since PAHs are likely coupled to the gas, PAH emission, when present, serves as confirmation of the presence of gas inside the gap. Unfortunately, we see no correlation between PAHs and accretion, both of which should trace gas within the holes. This is likely due to the dependence of PAH emission on strong UV flux, which is lacking from late-type stars.

\subsection{Notes on Individual Sources}

Sources 10, 12, 14, 20, 22, 23, 29, and 30 have neither $70 \mu \mathrm{m}$ nor $1.3 \mathrm{~mm}$ fluxes available. This makes it very difficult to distinguish between a low-mass disk and a more massive disk with an inner hole. The difference between the two lies in the presence of a substantial outer disk. The $70 \mu \mathrm{m}$ limits sometimes hint at the absence of such an outer disk but the limits are not 


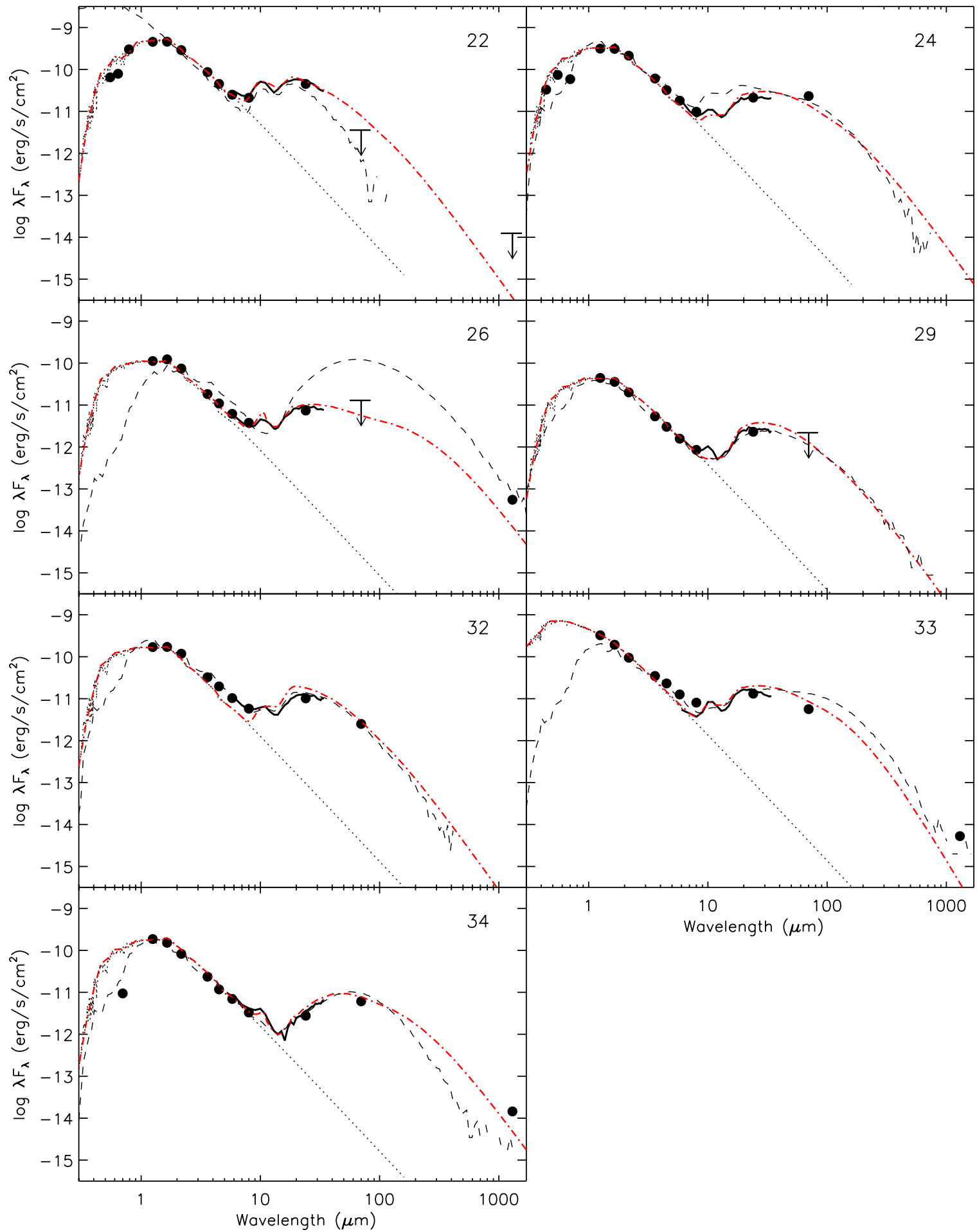

Figure 8. SEDs of the disks with holes in the sample. Points are dereddened fluxes from IRAC, MIPS, and IRAM. The thick solid line is the IRS spectrum, the dotted line is the stellar photospheric model, the red dash-dotted line is the best fit RADMC disk model, and the thin blank dashed line is the Robitaille model fit. The numbers give their identifications in Table 1.

(A color version of this figure is available in the online journal.)

always very stringent. Unless there is compelling evidence from the long-wavelength IRS spectra, we have taken the conservative view that these are likely low-mass disks and can be explained without needing to invoke an inner hole.

Two sources, 25 and 27, have extremely strong, extended $70 \mu \mathrm{m}$ emission and do not appear to be disks. In both cases, it is likely that the surrounding material has contaminated the long-wavelength photometry, producing these strange SEDs (see Figure 6). Source 25, DoAr 21, has been studied better than many of the other sources in this sample. Resolved imaging of $\mathrm{H}_{2}$ shows an extended ring structure at 73-219 AU away from the star (M. Hogerheijde et al. 2010, in preparation). Jensen et al. (2009) have speculated that much of the flux in long-wavelength unresolved photometry comes from the cloud material that the 


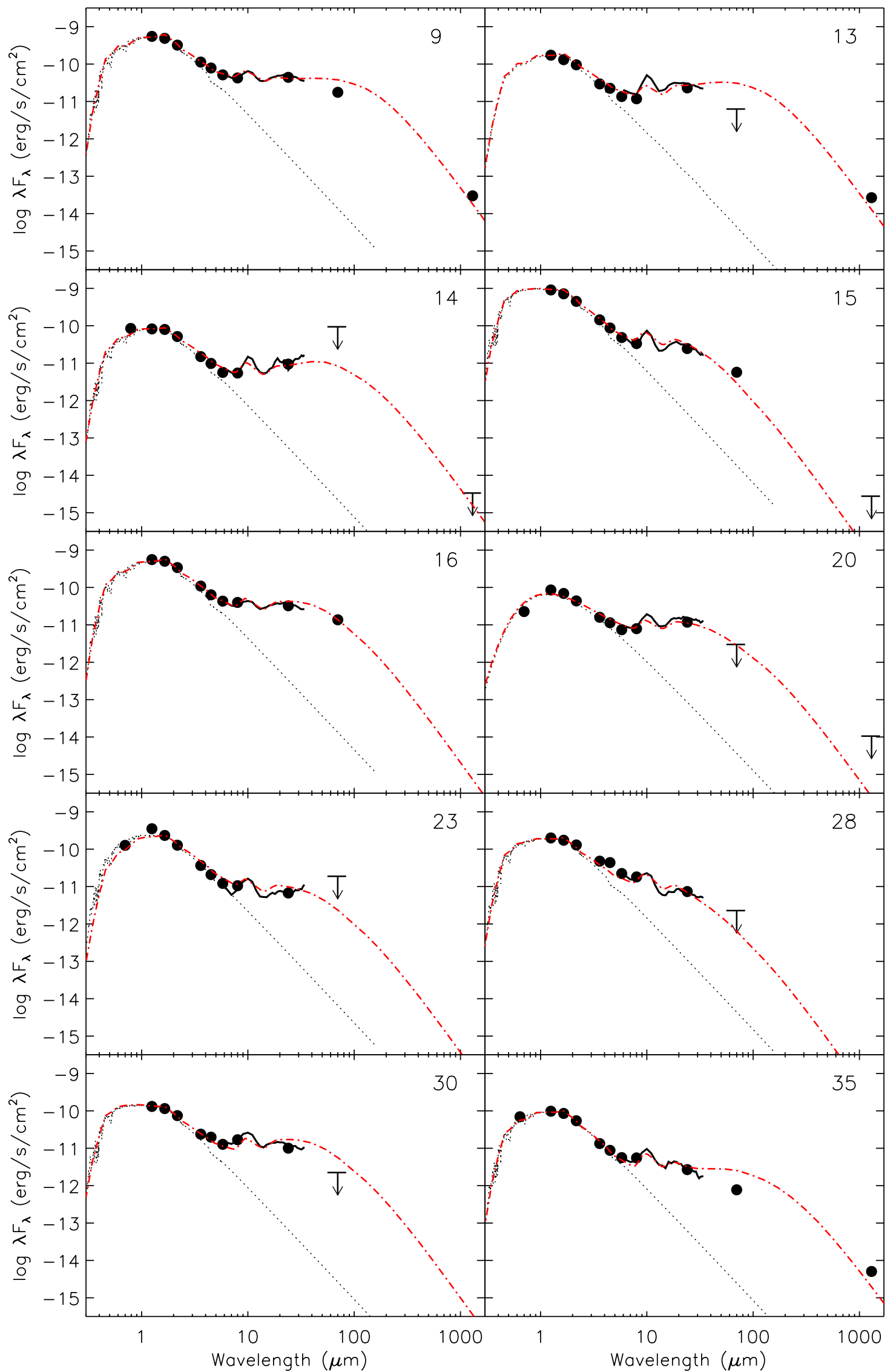

Figure 9. SEDs of the disks without holes in the sample. Points are dereddened fluxes from IRAC, MIPS, and IRAM. The thick solid line is the IRS spectrum, the dotted line is the stellar photospheric model and the red dash-dotted line is the best-fit RADMC disk model. The numbers give their identifications in Table 1.

(A color version of this figure is available in the online journal.) 


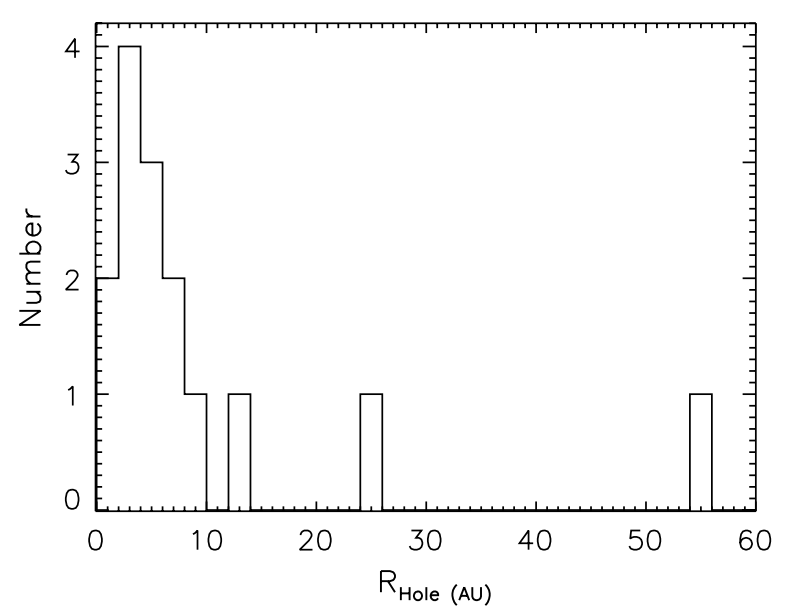

Figure 10. Inner disk radius histograms for the cold disks in the sample. The cold disks predominantly have inner holes with radii smaller than 10 AU. Many of the cold disks in the literature have larger hole sizes.

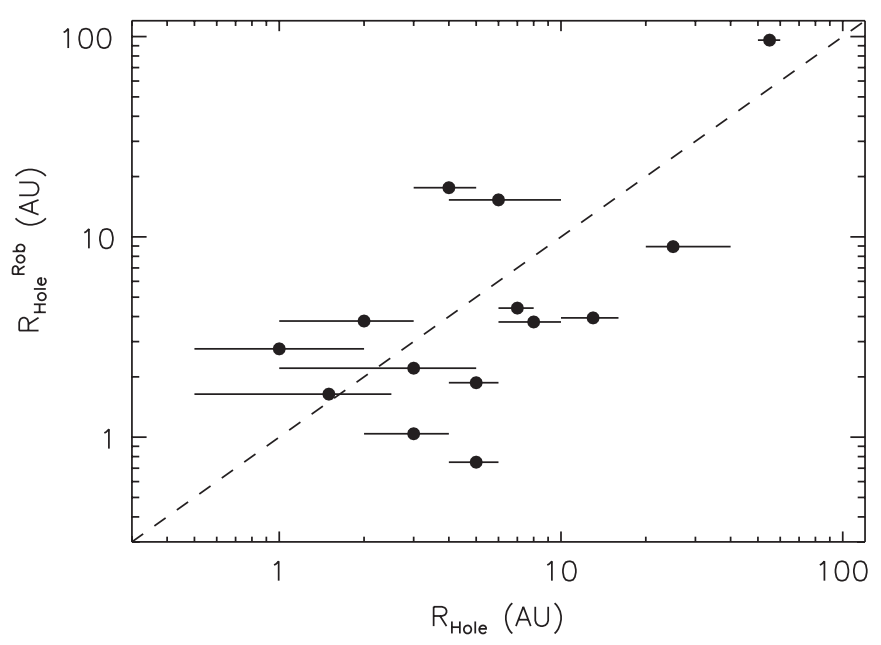

Figure 11. Comparison between hole radii computed with the RADMC models (Dullemond \& Dominik 2004) and with the Robitaille et al. (2007) disk model grid. Even with the large scatter, both quantities are correlated, which shows that the derived disk hole radius is model independent generally to within a factor of 2-3.

star is heating. Thus, while the SED looks compatible with a large inner hole, this might be due to excess material not directly associated with the star with long-wavelength fluxes being increasingly affected. Source 27 has a similar SED, which we suspect may arise from a similar physical situation. Both objects show exceptionally strong PAH emission (see Figure 13 and Section 4.3.2). Within a disk structure, the strong $70 \mu \mathrm{m}$ fluxes indicate large amounts of dust at 100-200 AU, and good SED fits required unphysical input parameters. Objects with very pronounced $70 \mu \mathrm{m}$ excesses but small $24 \mu \mathrm{m}$ excesses have been found to be produced by background contamination in the great majority of cases (Wahhaj et al. 2010). For these reasons, we have removed these objects from further study.

The $70 \mu \mathrm{m}$ flux of source 17 is affected by strong variable background emission, clearly visible in the c2d MIPS-70 image. Two photometric points are given in the SED (Figure 7) with the larger being the full aperture flux and the smaller a point-spread function aperture flux. The actual flux may be even smaller. The $24 \mu \mathrm{m}$ image does not show background emission and is in good agreement with IRS spectra. Although there is evidence for background contamination in the long-wavelength SED of this object, a sensible fit was possible with a disk with a hole.

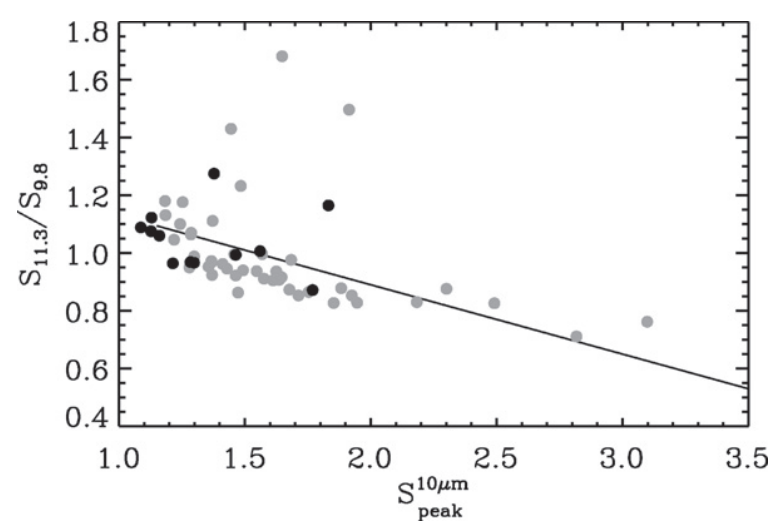

Figure 12. Shape-strength diagram for the $10 \mu \mathrm{m}$ silicate emission feature for all cold disks in this sample (filled dots), compared with those of the c2d IRS T Tauri sample (Olofsson et al. 2009, gray dots).

Sources $11,12,13,17$, and 18 have stellar temperatures lower than the Kurucz model grid and a blackbody is used instead.

Sources 18, 19, and 24 form a distinct subset of our sample. These three disks all have little to no near-IR excess but very flat disks longward of $8 \mu \mathrm{m}$. While there does appear to be a discontinuity between the inner and outer disks, the difference is not large. Despite the unknown disk mass, some degree of settling in the outer disk was required to reproduce the SED shape.

Finally, it is worth noticing that objects 2,13 , and 33 (15\% of the cold disk sample) show factor of 2 differences between IRAC and IRS fluxes in the overlapping wavelength range which are too large to be due to calibration errors. In the particular case of object 33, which has the largest difference, only the IRS spectrum hints at a possible inner hole, while the IRAC photometry could be easily explained without a hole. A careful check of the extraction of the IRS spectra and IRAC photometry found no evidence of extended emission, misalignment in the IRS observations, or any other instrumental reason for the discrepancy. Indeed, only 6 objects $(4 \%)$ out of the 147 IRS observed disks in the Serpens IRS survey have similar flux discrepancies between IRAC and IRS (I. Oliveira 2010, private communication). The IRAC observations were taken approximately two years before the spectroscopic ones (see Table 1). Similar scale variability was reported in Muzerolle et al. (2009) for another transitional disk. Such intrinsic variability might be the cause of this phenomenon but systematics between spectra and photometry could cause complications and further investigation is outside the scope of this work.

\section{DISCUSSION}

\subsection{Robust Selection Criteria}

\subsubsection{Spectroscopic Selection Criteria for Cold Disks}

Determining concrete selection criteria for cold disks is necessary to identify cold disks efficiently out of large Spitzer samples. Previously, cold disks have been identified in spectral samples using $30 \mu \mathrm{m} / 13 \mu \mathrm{m}$ flux ratios and slopes (e.g., Brown et al. 2007; Furlan et al. 2009). These two wavelengths bracket the rise in flux between an inner dust hole and substantial outer disk for holes with radii between 1 and 100 AU around stars with a representative range of stellar masses. The disks in this sample provide confirmation of this classification with $93 \%$ $(14 / 15)$ of the cold disks having $F_{30} / F_{13}$ flux ratios between 5 


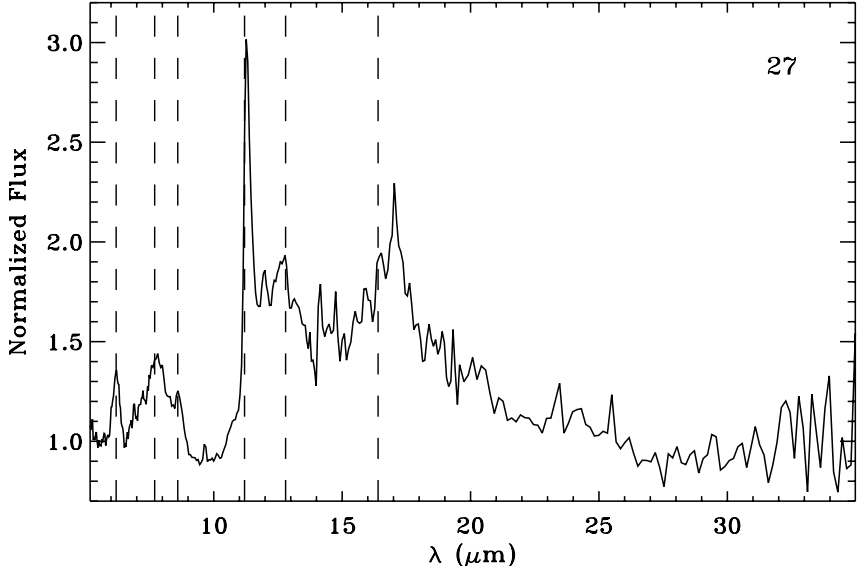

Figure 13. Continuum-subtracted $\mathrm{PAH}$ emission from object 27 . The vertical dashed lines show PAH features.

and 15 (see Figure 14). The only disk with $F_{30} / F_{13}<5$ is source 2, which has one of the smallest holes. Edge-on disks generally lie above $F_{30} / F_{13}=15$ but three overlap with the cold disk region. There is also no clean separation between the normal and cold disks at $F_{30} / F_{13}=5$. The $30 \mu \mathrm{m} / 13 \mu \mathrm{m}$ flux ratio has a crude correlation with larger hole sizes having larger flux ratios, but there is unfortunately no clear relation between the $30 \mu \mathrm{m} / 13 \mu \mathrm{m}$ flux ratios and the slope between the Spitzer 8 and $24 \mu \mathrm{m}$ photometry, particularly for cold disks. Thus, the 8-24 $\mu \mathrm{m}$ slopes and colors cannot be used as a proxy for estimating hole size, although they can be used to select cold disks, as we discuss in the following section.

\subsubsection{Photometric Selection Criteria}

To identify the cold disk candidates out of the YSO population, we tested several combinations of color-color diagrams with different Spitzer bands to determine the cleanest identification of disks with inner holes (cold disks). We included not only the results of the detailed SED fitting from Section 4.2 but also the literature information about cold disks in the $\mathrm{c} 2 \mathrm{~d}$ clouds, including two sources from Brown et al. (2007) and two sources from Andrews et al. (2009). The selection criteria proposed here can be applied to any Spitzer IRAC and MIPS YSO photometric sample of any star-forming region to identify the disks with inner holes.

The criteria are based on colors without dereddening as the extinction to many of the sources in the catalog is unknown. The effects of $A_{\mathrm{V}}=10$ are plotted as an arrow on the diagrams. The effects are small for extinctions less than 10. Analysis of the spectral sample based on dereddened colors makes little difference in the selection regions.

The cold disk candidate sample is defined with the following cuts in magnitudes, organized in two sections for two different types of objects (see the top left panel in Figure 15). Region A selects "clean" inner holes (i.e., disks for which there is no substantial excess in any IRAC band and the signature of an inner opacity hole). Region B selects disks with some excess disk flux in the IRAC bands. The definition of the boundaries is as follows:

$$
\begin{aligned}
& \text { Region A: } 0.0<[3.6]-[8.0]<1.1 ; \\
& 3.2<[8.0]-[24]<5.3, \\
& \text { Region B: } 1.1<[3.6]-[8.0]<1.8 ; \\
& 3.2<[8.0]-[24]<5.3 .
\end{aligned}
$$

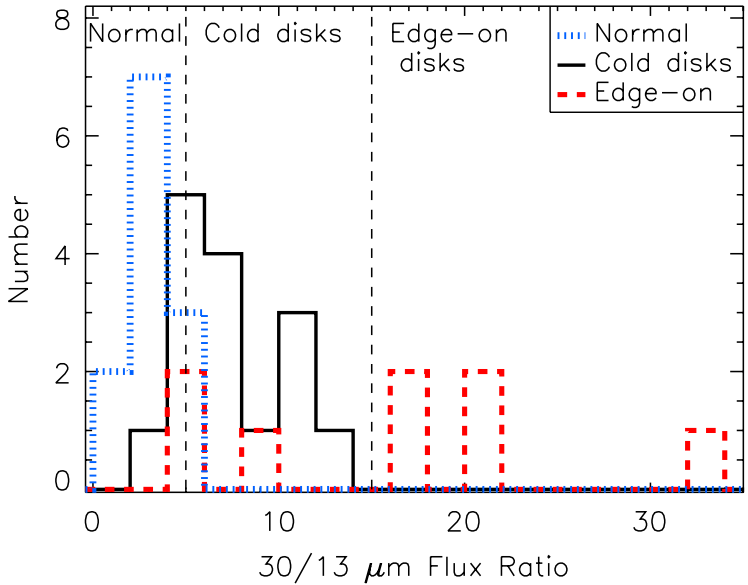

Figure 14. Histogram of $F_{30} / F_{13}$ ratios for the disks in this paper, with the definition of the boundaries by Brown et al. (2007) marked by vertical dashed lines for comparison. The region on the left is supposed to contain disks without holes, the middle region holds cold disks, and the right, edge-on disks. The actual distribution of these types of sources in our sample is shown for normal disks with the blue short-dashed line, cold disks with the solid black line, and edge-on disks with the red long-dashed line. There is significant overlap between all categories around $F_{30} / F_{13}$ of 5 but the general trends hold true in our sample.

(A color version of this figure is available in the online journal.)

Region B contains the four literature sources plus source 2, while the majority of the new cold disks $(13 / 15)$ lie in Region A. One possible explanation for this division is the focus of this work on looking for photospheric fluxes in the near-IR, while disks with large holes often have small near-IR excesses (e.g., Brown et al. 2007; Espaillat et al. 2007).

From the overall photometric catalog, the statistics on cold disks within the five c2d clouds can be determined, including sources not included in the spectroscopic follow up for a variety of reasons such as low fluxes. These disks are marked with small crosses in Figure 15. There are 43 cold disk candidates in Region A accounting for $4 \%$ of the total disk population in the $\mathrm{c} 2 \mathrm{~d}$ YSO catalog. Only one of the objects in that region for which we performed detailed SED modeling including the IRS spectrum was a false positive. This is object 25, DoAr 21, which has a cold disk-like SED but the $70 \mu \mathrm{m}$ flux may be strongly contaminated by cloud material (see Section 4.4 for further details). From the spectroscopically studied objects in Region A, 93\% are cold disks.

Region B is more complicated. While there are well studied, confirmed cold disks within this region, there are also objects which are not cold disks. Region B contains 181 YSOs in the $\mathrm{c} 2 \mathrm{~d}$ catalog, but fewer sources were selected for our study from this region. Of this small subsample and excluding the rising sources, for which it is not possible to determine the presence of an inner hole in the disk, only $42 \%$ of the studied disks turned out to be cold disks, so we estimate a contamination on the order of $58 \%$ in this region. These percentages have considerable uncertainty given the low-number statistics. Region B includes the disks WSB 60 and DoAr 44, for which inner holes of 20 and 33 AU, respectively, have been resolved with the SMA (Submillimeter Array), but whose SEDs show no detectable signature of these disks (Andrews et al. 2009). This implies that selecting purely on broadband colors will always be prone to uncertainty. Individual source modeling, spectroscopic studies, and non-SED-based searches are all needed to fully understand cold disk frequencies, particularly in this region of the color-color parameter space.

If we add together the 43 objects in Region A with $42 \%$ of the 181 objects in Region B, we have a total of 119 cold 

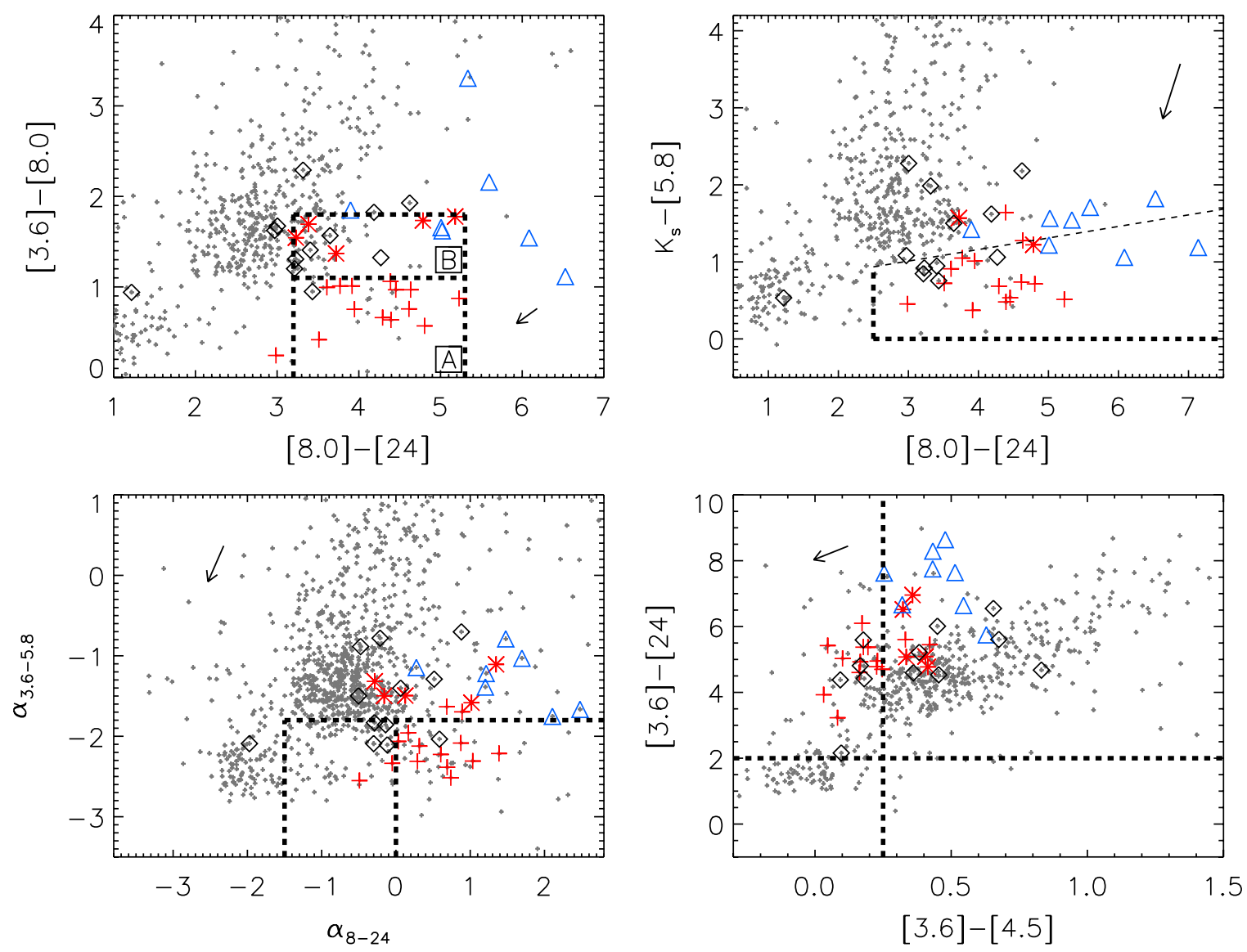

Figure 15. Selection criteria. Red stars and crosses are the spectroscopically confirmed cold disks, black diamonds are disks without holes, and blue triangles are edge-on disks. The gray crosses are all the sources in the c2d YSO catalog (Evans et al. 2009). Top left: our selection criteria. Regions A and B define the two selection criteria to identify cold disks in the photometric sample: the first one, represented with red crosses, selects "clean" inner holes, and the second one, represented with red stars, identifies cold disks with near-IR excess. Top right: selection criteria from Fang et al. (2009). Bottom left: selection criteria from Muzerolle et al. (2010). Bottom right: selection criteria from Cieza et al. (2010) with transitional disks according to their definitions in the upper left quadrant. Arrows show the effect of correcting for an extinction of $A_{\mathrm{V}}=10$.

(A color version of this figure is available in the online journal.)

disks in the sample (12\% of the YSO catalog of 1024 sources). Therefore, the fraction of $4 \%$ of cold disks quoted above should be interpreted as a safe lower limit to the actual population of disks with inner holes in the c2d YSO catalog. The analysis of this photometric sample is outside the scope of this paper and will be presented in a separate work. Considering the IRS flux-limited sample in Serpens where all disks have IRS spectra, we confirm the presence of eight cold disks, which account for $9 \%$ of the total (Oliveira et al. 2010). This range of cold disk frequencies of $4 \%-10 \%$ comes with some caveats. Transitional disks with very low mass outer disks are not included in this study (such as the ones discussed in, e.g., Najita et al. 2007; Cieza et al. 2007), but are often included in other transitional disk frequency statistics. Such disks may represent a later stage of cold disk evolution or a completely different evolutionary pathway. Disks with holes smaller than $1 \mathrm{AU}$ cannot be reliably identified. Disks with large holes but with some near-IR excess can also be difficult to identify from photometry.

A final consideration is the potential bias introduced by the c2d YSO selection criterion itself. The c2d YSO sample is biased against objects with photospheric colors and only includes sources with good detections at all IRAC bands plus at the MIPS $24 \mu \mathrm{m}$ band. This obviously imposes a bias which is difficult to determine when selecting transitional disks in regions with very high background emission, e.g., the IC 348 or $\rho$ Oph clusters. In any case, the effect does not seem to be a dominant one since most of the cold disk candidates found with the color selection proposed here are clustered in a similar manner as the rest of the YSOs, namely, around the dense high-background clusters, while we should see a lack of such objects in high-background areas otherwise.

\subsubsection{Comparison with Transitional Disk Selection Criteria from Other Papers}

Fang et al. (2009) also recently proposed color-color diagram cuts to select cold disks. Their criteria form a trapezoid in the $K-$ [5.8] versus [8.0] - [24] color-color space defined by $K-[5.8]>0,[8.0]-[24]>2.5$, and the last side by $K-[5.8]<(0.56+([8.0]-[24]) \times 0.15)$. In the top right panel of Figure 15, we evaluate these criteria based on our spectrally confirmed cold disks. Most of the disks in our sample fall within or close to this area. Of our cold disks, 13/15 are within the boundaries while the remaining two are only just above the upper sloped line. However, half $(6 / 12)$ of the disks without holes, as well as some (3/8) of the edge-on disks, also lie within this region. Cold disks can be preferentially selected by cutting higher in [8.0] $-[24]$ at $\sim 3.5$ instead of 2.5. This would exclude more of the candidates that do not have inner holes while including all the disks with holes and also have the benefit of having a greater separation from the bulk sample in the larger catalog (shown in the figure as small gray crosses). The region of [8.0] - [24] between 2.5 and 3.5 has the potential 
to include transitional disks with low-mass outer regions, but individual disk modeling is needed to confirm the nature of these sources. An upper limit in [8.0] - [24] is also needed to remove the edge-on disks, with [8.0] $-[24]<5$ producing a reasonably clean separation.

Muzerolle et al. (2010) use slopes in $\log \lambda F_{\lambda}$ versus $\log \lambda$ between $3.6 \mu \mathrm{m}$ and $5.8 \mu \mathrm{m}, \alpha_{3-5}$, versus $8 \mu \mathrm{m}$ to $24 \mu \mathrm{m}, \alpha_{8-24}$. They propose that all transitional disks lie below -1.8 in $\alpha_{3-5}$ with weak excess sources between $\alpha_{8-24}=-1.5$ and $\alpha_{8-24}=0$ and normal cold disks at $\alpha_{8-24}>0$. Our sources lying within the weak excess region were all found not to need holes to fit the SEDs. The normal cold disk region selects sources similar to Region A, but sources within Region B are not selected by the Muzerolle et al. (2010) criteria.

Cieza et al. (2010) adopt a wider definition of transitional disks (e.g., they include objects with very small $24 \mu \mathrm{m}$ excesses), and their selection criteria are therefore less useful for identifying cold disks. Transitional disks are taken to be in the quadrant defined by [3.6] $-[24]>2$ and [3.6] $-[4.5]<0.25$. Just over half (12/19) of the cold disks lie within the selected quadrant, with seven cold disks missed by this criterion (generally those with more massive outer disks). Almost half $(5 / 12)$ of the disks without holes lie within the region and in the exact same position as the disks with holes. These disks may be homologously depleted transitional disks. A fairly large fraction of the catalog's classical disk locus lies within the selected region and might lead to a large false detection rate of cold disks.

\subsection{Properties of the Cold Disks}

One of the most obvious characteristics of this cold disk sample is the relatively small hole sizes (see the distribution in Figure 10). Many of the IRS-selected cold disks in the literature (e.g., Calvet et al. 2005; Brown et al. 2007; Espaillat et al. 2007; Kim et al. 2009) have large holes with radii greater than 10 AU. Of the fifteen cold disks examined here, only three have hole sizes larger than $10 \mathrm{AU}$ while an additional four large hole sources within the clouds are identified in the literature (Brown et al. 2007; Andrews et al. 2009). This may indicate that cold disks with very large holes ( $>10 \mathrm{AU})$ are intrinsically rarer than disks with smaller holes. Large hole sizes are easy to see from the IRS spectra but may not be as obvious from photometry. One complication is that disks with large inner holes often have some near-IR excess making the disks difficult to identify from IRAC colors. Another possible complication arises from the different disk models and methods used to determine the inner disk hole radii. In particular, the possibility cannot be ruled out that the RADMC models, which do not include viscous heating due to accretion, tend to produce smaller inner holes than other accreting disk models used by, e.g., Calvet et al. (2002). On the other hand, the hole radii determined with the same models and methods by Brown et al. (2007) were confirmed by the direct SMA observations of three disks by Brown et al. (2009). Finally, the similarity in terms of IR SEDs of the two millimeterdiscovered cold disks in Ophiuchus (Andrews et al. 2009) to classical disks means millimeter, as well as infrared, surveys, would be needed to ensure that all cold disks were found and to robustly confirm this trend. The differences in the definition of transitional disks between the works of Najita et al. (2007) and Cieza et al. (2008) were also extensively discussed in Cieza et al. (2010).

We find a statistically significant positive correlation between disk mass and hole size (see Figure 16). The correlation has a Pearson's correlation coefficient of 0.6 and is statistically

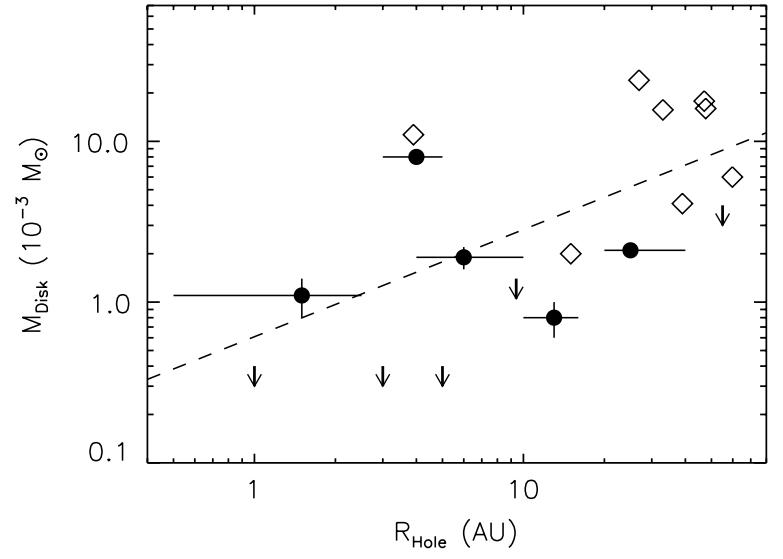

Figure 16. Correlation between hole size and disk mass. Dots and upper limits represent sources with millimeter fluxes from this work, while the diamonds are the cold disks with millimeter-measured disk masses from Kim et al. (2009) and Brown et al. (2007). To minimize systematic differences, disk masses for sources from Kim et al. (2009) have been recalculated using the $1.3 \mathrm{~mm}$ fluxes in Andrews \& Williams (2005) and the conversion in Section 4.1. The correlation has a Pearson's correlation coefficient of 0.6 and is statistically significant on the $99 \%$ level including the upper limits and $90 \%$ level with only the detected sources. The systematically lower disk masses in this survey might explain the generally smaller hole sizes than previously published transitional disks.

significant on the $99 \%$ level including upper limits on disk mass and on the $90 \%$ level with only detected sources. To minimize systematic differences, disk masses for sources from Kim et al. (2009) have been recalculated using the $1.3 \mathrm{~mm}$ fluxes in Andrews \& Williams (2005) and the flux to disk mass conversion in Section 4.1. The dependence of hole size on disk mass may point to a gravitational process, with more massive disks more likely to form more massive planets in larger orbits. The masses of transitional disks relative to normal disks remain unclear. Najita et al. (2007) find that transitional disks have larger disk masses for a sample of transitional disks in Taurus, although their disk classification method is different. Cieza et al. (2008) find very small disk masses for a sample of non-accreting WTTs. This trend may help explain the different results of the studies as Najita et al. (2007) focused mainly on cold disks from the literature which generally have large holes and are often accreting, while Cieza et al. (2008) were likely more sensitive to disks with smaller holes and no accretion.

Kim et al. (2009) find a correlation between hole size and stellar mass in their transitional disk sample in Chamaeleon and Taurus. Unfortunately, our sample is too similar in stellar mass to accurately test this trend and there is no correlation found from our sample alone. However, when combining our sample with the Kim et al. (2009) sample (see Figure 17), a correlation between the size of the inner holes and the stellar masses is retained. The disks from Brown et al. (2007) are in agreement with the Kim et al. (2009) trend, while the disks in this paper generally lie below the trend line, indicating smaller hole sizes. This may be partly due to the generally lower disk masses in this sample leading to smaller hole sizes based on the correlation in Figure 16.

Perhaps surprisingly, we see no trends with $L_{\text {Disk }} / L_{*}$, a measure of overall disk evolution (see Table 7). $L_{\text {Disk }} / L_{*}$ measures the integrated infrared excess normalized by the stellar luminosity and primarily traces the total grain surface area that is reprocessing stellar light. As disks become more tenuous, settle, and disappear, the strength of the disk luminosity should decline. This measure is commonly used in debris disk studies 


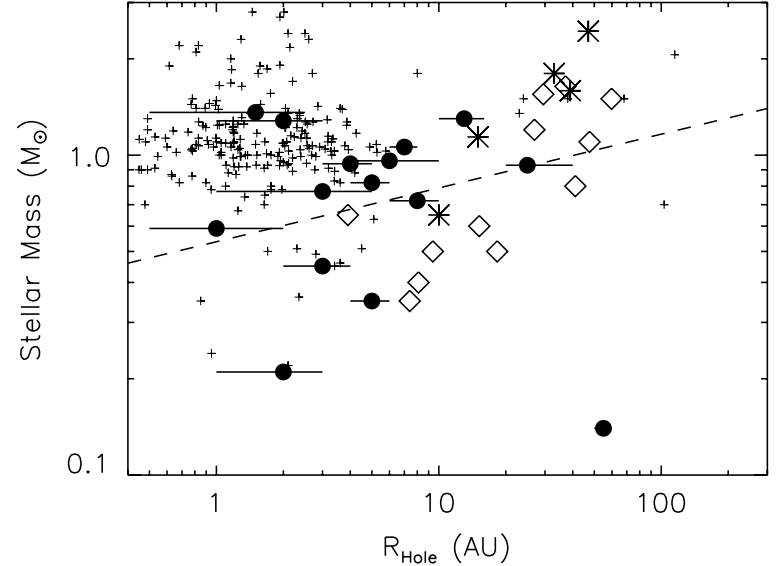

Figure 17. Relationship between the size of the inner disk hole and the mass of the central star. Solid circles represent our sample, while stars and diamonds are transitional disks from Brown et al. (2007) and Kim et al. (2009), respectively. The dotted line is the best linear fit to the data. The small crosses mark the semimajor axis of all exoplanets in the http://exoplanet.eu database as of 2009 December, compared to the mass of their parent stars.

(e.g., Morales et al. 2009). There is no correlation with hole size nor are there significant differences in $L_{\text {Disk }} / L_{*}$ between the sources with and without holes in this sample. Therefore, if $L_{\text {Disk }} / L_{*}$ can indeed be used as a probe for disk flaring, then this points to a distinct lack of settling in the outer regions of cold disks. Alternatively, this might also imply that $L_{\text {Disk }} / L_{*}$ is not especially sensitive to the changes in disk flaring while the outer disk keeps being optically thick.

The majority $(9 / 12,75 \%)$ of the cold disks with $\mathrm{H} \alpha$ spectra are accreting. Accretion is a particularly interesting diagnostic in transitional disks as it indicates material inside the hole. Large fractions of accreting cold disks indicate that in many cases gas must be flowing through the hole as any inner gas reservoir would drain quickly. This is in contrast with the results by Najita et al. (2007), who report lower mass accretion rates in transitional disks with respect to the CTTSs in Taurus, although they use a completely different definition of transitional disk from the one used here.

Silicate emission probes dust properties such as grain size and crystallinity. Silicate emission, especially the distinct $10 \mu \mathrm{m}$ silicate stretching band, is seen from all but one of the cold disks. However, the presence and intensity of the $10 \mu \mathrm{m}$ feature decrease substantially for inner hole radii larger than 7-10 AU (Figure 18). This is partly to be expected as dust-depleted inner holes result in a lack of silicate grains at the correct disk temperatures to produce the features. A large fraction of the cold disks show crystalline features at wavelengths longer than $20 \mu \mathrm{m}$ indicating processing that likely occurred in the inner regions of the disks where temperature and densities are high. These regions are now largely depleted in the cold disks but dust mixing, fragmentation of larger bodies, or alternative formation routes have resulted in detectable amounts remaining further out in the disk.

\subsection{The Possible Origins of the Inner Holes}

The origins of inner holes are still under debate among several theories developed to explain inner holes and gaps in protoplanetary disks, including (1) EUV photoevaporation of the inner disk (Clarke et al. 2001; Alexander et al. 2006), (2) settling and coagulation of dust into large particles (Tanaka et al. 2005; Dullemond \& Dominik 2005), or (3) by the dynamical

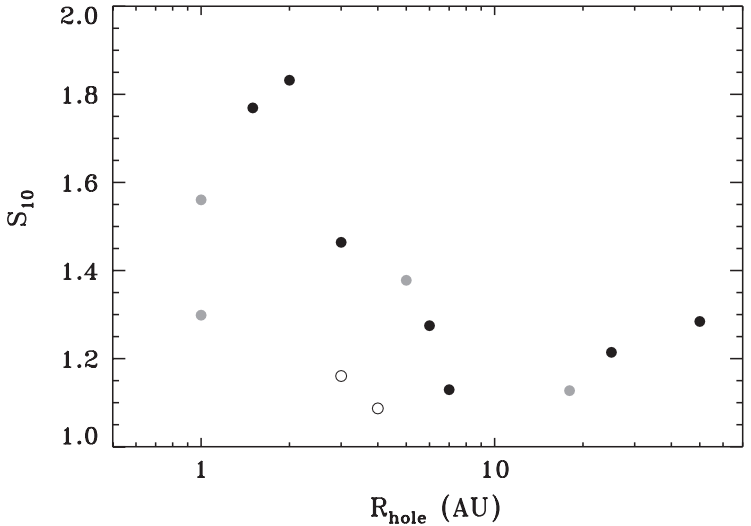

Figure 18. Relationship between the size of the inner disk hole and the intensity of the $10 \mu \mathrm{m}$ feature. Solid, gray, and open circles represent c2d accreting, non-accreting cold disks, and objects for which we do not yet have accretion information, respectively.

perturbation of unresolved companions, both stars (Artymowicz \& Lubow 1994) and planets (Quillen et al. 2004; Varnière et al. 2006). It is likely that all are present on some level but it is not clear which are dominant in which systems (Cieza et al. 2010; Sicilia-Aguilar et al. 2010).

For photoevaporation, an inner hole occurs when the photoevaporation rate driven by the EUV ionizing flux from the central star matches the viscous accretion rate (Alexander et al. 2006). FUV photoevaporation also plays a significant role in dissipating disks but predominantly removes less bound gas from the outer regions and would therefore not create cold disk SEDs (Gorti \& Hollenbach 2009). Photoevaporation is most effective when accretion rates are low and would result in no gas or dust close to the star within 0.1-1 Myr, depending on assumed disk properties such as viscosity and treatment of the UV ionizing flux (Alexander et al. 2006; Gorti \& Hollenbach 2009). The presence of accretion in such a large fraction $(75 \%)$ of this sample discards photoevaporation as the primary origin. Some disks with inner holes do show evidence for photoevaporation based on $[\mathrm{Ne}$ II] observations but the implied mass-loss rates are too low to disperse the disk in under $10 \mathrm{Myr}$ (Pascucci \& Sterzik 2009). The disk masses of the remaining $25 \%$ are typically low enough that photoevaporation could be the cause of the inner hole.

Accelerated grain growth in the inner regions could produce cold disk SEDs as dust grains become too big to be observed (Tanaka et al. 2005). Models predict that dust particles grow and settle toward the dense disk midplane, where they may stick together to form planetesimals (Weidenschilling 2000). Growth is likely preferential in the inner disk, and these larger bodies will grow and can eventually accrete a large fraction of the surrounding gas to become giant planets (Bryden et al. 1999; Wuchterl et al. 2000 and references therein). In this scenario, grain growth and settling would happen throughout the disk but with faster timescales in the inner region. If grain growth is accompanied by settling throughout the disk, decreasing $L_{\text {Disk }} / L_{*}$ with increasing hole size would be expected. We do not see this in our sample, making this unlikely to be the dominant scenario, although such a correlation for a subsample of the sources may be masked by short inner disk settling timescales or objects formed by different mechanisms. Another potential complication is that coagulation in the inner regions would not affect $L_{\text {Disk }} / L_{*}$ in the absence of settling in the outer disk. Holes created via grain growth are likely to have more gradual hole edges compared to holes created by companions. 
Binary companions are a possible explanation for cold disk SEDs. Some candidate cold disks, such as CoKu Tau 4, have later been determined to be circumbinary (Ireland \& Kraus 2008). The issue clearly requires further high-resolution imaging of these sources (e.g., Marois et al. 2008; Kalas et al. 2008; Lagrange et al. 2009) or radial velocity measurements to confirm the possible presence of stellar or substellar-mass companions. However, this is time consuming for large samples and may not even be possible for more distant regions or planets at larger radii which require long-term monitoring. Preliminary results of binary searches show that close binary companions often result in the complete dissipation of the disk and that circumbinary "transition" disks may not be common (Kraus et al. 2009; Pott et al. 2010). If stellar companions carve out inner holes, accretion may either continue or cease depending on the separation and mass ratio of the two stars (Artymowicz \& Lubow 1996; Ireland \& Kraus 2008). On the other hand, if a planet carves out an inner hole, accretion can continue at a reduced rate aided by mechanisms such as the magnetorotational instability (Chiang \& Murray-Clay 2007).

Inner holes created by planets remain a popular and exciting explanation. Of the scenarios described, this is the most difficult to positively confirm or deny. Planets are faint so direct observation is difficult, while their small masses leave weaker gravitational signatures on the disk than stellar companions. The inner hole radii are compatible with the distribution of the exoplanet semimajor axis, as found in the latest exoplanet database as of 2009 December $^{16}$ (Figure 17). As the exoplanets likely formed in similar disks before dissipation, it is likely that there are young exoplanets in some disks at these types of radii, but whether they are responsible for the cold disk signatures discussed in this paper remains to be determined.

\section{SUMMARY}

The main results of this work are summarized as follows.

1. Optical spectra, 2MASS, and Spitzer photometry, millimeter continuum observations, and Spitzer/IRS 5-35 $\mu \mathrm{m}$ spectra of a sample of 35 cold disk candidates selected from c $2 \mathrm{~d}$ photometry are presented and analyzed.

2. Out of the 35 objects in the initial sample, SED modeling identifies 15 as disks with inner holes, which we call "cold disks," following the c2d convention (Brown et al. 2007). Of the remaining sources, ten could be modeled without holes, eight are edge-on disks, and two have SEDs strongly contaminated by cloud material.

3. The color cuts $0.0<[3.6]-[8.0]<1.1$ and $3.2<$ $[8.0]-[24]<5.3$ identify most cold disks from this sample $(\sim 80 \%)$, in particular those with the cleanest inner holes. Extension of the [3.6] - [8.0] color cut to 1.8 recovers some objects with small near-IR excesses and large holes, but contains contamination from disks without holes. Out of the large c2d YSO sample, $\sim 12 \%$ of the disks are estimated to be cold disks based on these selection criteria.

4. We evaluated the criteria of Fang et al. (2009), Muzerolle et al. (2010), and Cieza et al. (2010) and suggest improvements based on our spectroscopic study.

5. The cold disks presented here have small hole sizes, generally less than 10 AU. This distribution agrees more with exoplanet orbit radii than the large hole sizes of most cold disks in the literature.

\footnotetext{
16 http://www.exoplanets.eu
}

6. A large fraction (75\%) of the cold disks are accreting, suggesting that gas is flowing through the dust depleted hole. This large fraction of accreting disks is not in agreement with the dominant hole origin being photoevaporation.

7. Hole size correlates with disk mass, with more massive disks tending to have larger holes.

8. The sizes of the inner holes scale linearly with the stellar mass although with a large spread.

9. The $10 \mu \mathrm{m}$ silicate features in the sample show substantial grain growth. The $10 \mu \mathrm{m}$ silicate emission feature strength decreases drastically with respect to the continuum for inner holes larger than $\sim 7$ AU. Some $(33 \%-60 \%)$ of the cold disks show long-wavelength crystalline features, indicating that mixing from the inner regions where crystallization occurs to outside the inner hole region must be efficient. Only two sources $(\sim 13 \%)$ show PAH emission.

Support for this work, part of the Spitzer Space Telescope Legacy Science Program, was provided by NASA through contract numbers 1224608 for c2d, 1288664 for GO3 IRS cold disks, 1256316 and 1230780 issued by the Jet Propulsion Laboratory, and for the California Institute of Technology under NASA contract 1407. Astrochemistry at Leiden was supported by a NWO Spinoza and NOVA grant, and by the European Research Training Network "The Origin of Planetary Systems" (PLANETS, contract number HPRN-CT-2002-00308).

Facilities: Spitzer (IRAC, MIPS, IRS), CAO:2.2m (CAFOS), ING:Herschel (ISIS, WYFFOS), ING:Newton (IDS), PO:1.5m (DBSP), IRAM:30m (MAMBO)

\section{REFERENCES}

Acke, B., \& van den Ancker, M. E. 2004, A\&A, 426, 151

Alcalá, J. M., et al. 2008, ApJ, 676, 427

Alexander, R. D., Clarke, C. J., \& Pringle, J. E. 2006, MNRAS, 369, 216

Allers, K. N., Kessler-Silacci, J. E., Cieza, L. A., \& Jaffe, D. T. 2006, ApJ, 644, 364

André, P., Montmerle, T., Feigelson, E. D., \& Steppe, H. 1990, A\&A, 240, 321

Andrews, S. M., \& Williams, J. P. 2005, ApJ, 631, 1134

Andrews, S. M., \& Williams, J. P. 2007, ApJ, 671, 1800

Andrews, S. M., Wilner, D. J., Hughes, A. M., Qi, C., \& Dullemond, C. P. 2009, ApJ, 700, 1502

Artymowicz, P., \& Lubow, S. H. 1994, ApJ, 421, 651

Artymowicz, P., \& Lubow, S. H. 1996, ApJ, 467, L77

Aspin, C., Sandell, G., \& Russell, A. P. G. 1994, A\&AS, 106, 165

Baraffe, I., Chabrier, G., Allard, F., \& Hauschildt, P. H. 1998, A\&A, 337, 403

Beckwith, S. V. W., Sargent, A. I., Chini, R. S., \& Guesten, R. 1990, AJ, 99, 924

Bingham, R. G., Gellatly, D. W., Jenkins, C. R., \& Worswick, S. P. 1994, Proc. SPIE, 2198, 56

Bouwman, J., et al. 2008, ApJ, 683, 479

Brown, J. M., Blake, G. A., Qi, C., Dullemond, C. P., \& Wilner, D. J. 2008, ApJ, 675, L109

Brown, J. M., et al. 2007, ApJ, 664, L107 (B07)

Brown, J. M., et al. 2009, ApJ, 704, 496

Bryden, G., Chen, X., Lin, D. N. C., Nelson, R. P., \& Papaloizou, J. C. B. 1999, ApJ, 514, 344

Calvet, N., et al. 2002, ApJ, 568, 1008

Calvet, N., et al. 2005, ApJ, 630, L185

Carpenter, J. M., Mamajek, E. E., Hillenbrand, L. A., \& Meyer, M. R. 2009, ApJ, 705,1646

Chapman, N. L., Mundy, L. G., Lai, S., \& Evans, N. J. 2009, ApJ, 690, 496

Chen, C. H., et al. 2006, ApJS, 166, 351

Chiang, E. I., \& Goldreich, P. 1997, ApJ, 490, 368

Chiang, E., \& Murray-Clay, R. 2007, Nat. Phys., 3, 604

Cieza, L., et al. 2007, ApJ, 667, 308

Cieza, L. A., Swift, J. J., Mathews, G. S., \& Williams, J. P. 2008, ApJ, 686, L115

Cieza, L. A., et al. 2010, ApJ, 712, 925

Clarke, C. J., Gendrin, A., \& Sotomayor, M. 2001, MNRAS, 328, 485 
Comerón, F., Spezzi, L., \& López Martí, B. 2009, A\&A, 500, 1045

Currie, T., et al. 2009, ApJ, 698, 1

Dahm, S. E., \& Carpenter, J. M. 2009, AJ, 137, 4024

Dolidze, M. V., \& Arakelyan, M. A. 1959, Astron. Zh., 36, 444

Dullemond, C. P., \& Dominik, C. 2004, A\&A, 417, 159

Dullemond, C. P., \& Dominik, C. 2005, A\&A, 434, 971

Dullemond, C. P., \& Dominik, C. 2008, A\&A, 487, 205

Dullemond, C. P., Hollenbach, D., Kamp, I., \& D’Alessio, P. 2007, in Protostars and Planets V, ed. B. Reipurth, D. Jewitt, \& K. Keil (Tucson, AZ: Univ. Arizona Press), 555

Ercolano, B., Clarke, C. J., \& Robitaille, T. P. 2009, MNRAS, 394, L141

Espaillat, C., Calvet, N., Luhman, K. L., Muzerolle, J., \& D'Alessio, P. 2008, ApJ, 682, L125

Espaillat, C., et al. 2007, ApJ, 670, L135

Evans, N. J., II., et al. 2003, PASP, 115, 965

Evans, N. J., et al. 2009, ApJS, 181, 321

Fang, M., et al. 2009, A\&A, 504, 461

Flaherty, K. M., et al. 2007, ApJ, 663, 1069

Forrest, W. J., et al. 2004, ApJS, 154, 443

Furlan, E., et al. 2006, ApJS, 165, 568

Furlan, E., et al. 2009, ApJ, 703, 1964

Gagné, M., Skinner, S. L., \& Daniel, K. J. 2004, ApJ, 613, 393

Geers, V. C., et al. 2006, A\&A, 459, 545

Geers, V. C., et al. 2007a, A\&A, 469, L35

Geers, V. C., et al. 2007b, A\&A, 476, 279

Getman, K. V., et al. 2002, ApJ, 575, 354

Gorti, U., \& Hollenbach, D. 2009, ApJ, 690, 1539

Gutermuth, R. A., et al. 2004, ApJS, 154, 374

Habart, E., Natta, A., Testi, L., \& Carbillet, M. 2006, A\&A, 449, 1067

Haisch, K. E., Jr., Lada, E. A., \& Lada, C. J. 2001, ApJ, 553, L153

Hartigan, P. 1993, AJ, 105, 1511

Hartmann, L. 1998, Accretion Processes in Star Formation (Cambridge: Cambridge Univ. Press)

Harvey, P., et al. 2007, ApJ, 663, 1149

Hauschildt, P. H., Allard, F., Ferguson, J., Baron, E., \& Alexander, D. R. 1999, ApJ, 525, 871

Henning, T., Pfau, W., Zinnecker, H., \& Prusti, T. 1993, A\&A, 276, 129

Herbig, G. H., \& Bell, K. R. (ed.) 1988, Lick Obs. Bull. 1111, Catalog of Emission-line Stars of the Orion Population (Santa Cruz, CA: Lick Observatory)

Hernández, J., et al. 2007, ApJ, 662, 1067

Hughes, A. M., et al. 2007, ApJ, 664, 536

Hughes, A. M., et al. 2009, ApJ, 698, 131

Ireland, M. J., \& Kraus, A. L. 2008, ApJ, 678, L59

Jayawardhana, R., Coffey, J., Scholz, A., Brandeker, A., \& van Kerkwijk, M. H. 2006, ApJ, 648, 1206

Jensen, E. L. N., Cohen, D. H., \& Gagné, M. 2009, ApJ, 703, 252

Jørgensen, J. K., et al. 2006, ApJ, 645, 1246

Jura, M., et al. 2004, ApJS, 154, 453

Kalas, P., et al. 2008, Science, 322, 1345

Kenyon, S. J., \& Bromley, B. C. 2009, ApJ, 690, L140

Kenyon, S. J., \& Hartmann, L. 1995, ApJS, 101, 117

Kessler-Silacci, J., et al. 2006, ApJ, 639, 275

Kim, K. H., et al. 2009, ApJ, 700, 1017

Koike, C., Shibai, H., \& Tuchiyama, A. 1993, MNRAS, 264, 654

Kraus, A. L., Ireland, M. J., Martinache, F., \& Lloyd, J. P. 2009, in AIP Conf. Proc. 1094, Cool Stars, Stellar Systems and the Sun, ed. E. Stempels (Melville, NY: AIP), 453

Krautter, J., et al. 1997, A\&AS, 123, 329

Kreysa, E., Gemünd, H.-P., Raccanelli, A., Reichertz, L. A., \& Siringo, G. 2002, in AIP Conf. Proc. 616, Experimental Cosmology at Millimetre Wavelengths, ed. M. de Petris \& M. Gervasi (Melville, NY: AIP), 262

Kurosawa, R., Harries, T. J., \& Symington, N. H. 2006, MNRAS, 370, 580

Lada, C. J., et al. 2006, AJ, 131, 1574

Lagrange, A.-M., et al. 2009, A\&A, 493, L21

Lahuis, F., et al. 2007, ApJ, 665, 492

Leggett, S. K. 1992, ApJS, 82, 351

Liu, C.-P., Zhang, C.-S., \& Kimura, H. 1980, Acta Astron. Sin., 21, 354

Low, F. J., et al. 2005, ApJ, 631, 1170

Luhman, K. L., Briceno, C., Rieke, G. H., \& Hartmann, L. 1998, ApJ, 493, 909
Luhman, K. L., et al. 2003, ApJ, 590, 348

Marois, C., et al. 2008, Science, 322, 1348

Mathis, J. S., Rumpl, W., \& Nordsieck, K. H. 1977, ApJ, 217, 425

McClure, M. 2009, ApJ, 693, L81

Merín, B., et al. 2008, ApJS, 177, 551

Montes, D., Martin, E. L., Fernandez-Figueroa, M. J., Cornide, M., \& de Castro, E. 1997, A\&AS, 123, 473

Mora, A., et al. 2001, A\&A, 378, 116

Morales, F. Y., et al. 2009, ApJ, 699, 1067

Muench, A. A., Lada, C. J., Luhman, K. L., Muzerolle, J., \& Young, E. 2007, AJ, 134,411

Muzerolle, J., Allen, L. E., Megeath, S. T., Hernández, J., \& Gutermuth, R. A. 2010, ApJ, 708, 1107

Muzerolle, J., Hillenbrand, L., Calvet, N., Briceño, C., \& Hartmann, L. 2003, ApJ, 592, 266

Muzerolle, J., et al. 2009, ApJ, 704, L15

Najita, J. R., Strom, S. E., \& Muzerolle, J. 2007, MNRAS, 378, 369

Natta, A., et al. 2004, A\&A, 424, 603

Nguyen, D. C., Scholz, A., van Kerkwijk, M. H., Jayawardhana, R., \& Brandeker, A. 2009, ApJ, 694, L153

Nuernberger, D., Chini, R., \& Zinnecker, H. 1997, A\&A, 324, 1036

Oke, J. B., \& Gunn, J. E. 1982, PASP, 94, 586

Oliveira, I., et al. 2009, ApJ, 691, 672

Oliveira, I., et al. 2010, ApJ, 714, 778

Olofsson, J., et al. 2009, A\&A, 507, 327

Osterloh, M., \& Beckwith, S. V. W. 1995, ApJ, 439, 288

Padgett, D. L., et al. 1999, AJ, 117, 1490

Padgett, D. L., et al. 2006, ApJ, 645, 1283

Pascucci, I., \& Sterzik, M. 2009, ApJ, 702, 724

Piétu, V., Dutrey, A., Guilloteau, S., Chapillon, E., \& Pety, J. 2006, A\&A, 460, L43

Pollack, J. B., et al. 1996, Icarus, 124, 62

Pontoppidan, K. M., et al. 2008, ApJ, 684, 1323

Pott, J., et al. 2010, ApJ, 710, 265

Preibisch, T., Stanke, T., \& Zinnecker, H. 2003, A\&A, 409, 147

Preibisch, T., \& Zinnecker, H. 2001, AJ, 122, 866

Quillen, A. C., Blackman, E. G., Frank, A., \& Varnière, P. 2004, ApJ, 612, L137

Reipurth, B., Pedrosa, A., \& Lago, M. T. V. T. 1996, A\&AS, 120, 229

Robitaille, T. P., Whitney, B. A., Indebetouw, R., \& Wood, K. 2007, ApJS, 169 , 328

Salyk, C., Blake, G. A., Boogert, A. C. A., \& Brown, J. M. 2009, ApJ, 699, 330 Schwartz, R. D. 1977, ApJS, 35, 161

Sicilia-Aguilar, A., Hartmann, L. W., Hernández, J., Briceño, C., \& Calvet, N. 2005, AJ, 130, 188

Sicilia-Aguilar, A., Henning, T., \& Hartmann, L. W. 2010, ApJ, 710, 597

Sicilia-Aguilar, A., et al. 2008, ApJ, 687, 1145

Siess, L., Dufour, E., \& Forestini, M. 2000, A\&A, 358, 593

Skrutskie, M. F., et al. 1990, AJ, 99, 1187

Spezzi, L., Merín, B., Oliveira, I., van Dishoeck, E. F., \& Brown, J. M. 2010, A\&A, 513, A38

Spezzi, L., et al. 2008, ApJ, 680, 1295

Strom, K. M., Strom, S. E., Edwards, S., Cabrit, S., \& Skrutskie, M. F. 1989, AJ, 97,1451

Sylvester, R. J., Skinner, C. J., Barlow, M. J., \& Mannings, V. 1996, MNRAS, 279,915

Tanaka, H., Himeno, Y., \& Ida, S. 2005, ApJ, 625, 414

Tielens, A. G. G. M. 2008, ARA\&A, 46, 289

van Boekel, R., et al. 2005, A\&A, 437, 189

Varnière, P., Blackman, E. G., Frank, A., \& Quillen, A. C. 2006, ApJ, 640, 1110

Wahhaj, Z., et al. 2010, ApJ, submitted

Walawender, J., Bally, J., \& Reipurth, B. 2005, AJ, 129, 2308

Watson, D. M., et al. 2009, ApJS, 180, 84

Weidenschilling, S. J. 2000, Space Sci. Rev., 92, 295

Weingartner, J. C., \& Draine, B. T. 2001, ApJ, 548, 296

White, R. J., \& Basri, G. 2003, ApJ, 582, 1109

White, R. J., \& Hillenbrand, L. A. 2004, ApJ, 616, 998

Wuchterl, G., Guillot, T., \& Lissauer, J. J. 2000, in Protostars and Planets IV, ed. V. Mannings, A. P. Boss, \& S. S. Russell (Tucson, AZ: Univ. Arizona Press), 1081

Zylka, R. 1998, MOPSI Users Manual (Grenoble: IRAM) 